\title{
RECHERCHES
}

SUR LES

NERFS CÉRÉBRAUX ET LA MUSCULATURE CÉPHALIQUE

$\mathrm{DE}$

\section{SILURUS GLANIS}

PAR

\section{Marc J U G E}

Avec les planches 1 à $\bar{~}$

\section{INTRODUCTION}

Ce travail a été fait dans le Laboratoire de Zoologie et d'Anatomie comparée de l'Université de Genève. Qu'il me soit permis d'adresser à M. le professeur YUNG l'expression de ma reconnaissance pour la bienveillance qu'il a manifestée à mon égard et tout particulièrement pour la libéralité avec laquelle il m'a procuré nombre d'ouvrages importants. J'ai aussi à remercier vivement M. le professeur BEDOT, directeur du Musée d'histoire naturelle, auquel je dois unẹ partie du matériel employé et la connaissance de plusieurs mémoires.

Au début, ces recherches devaient porter sur les nerfs cérébraux d'un grand nombre de Téléostéens, mais, après avoir poussé assez loin l'étude de quelques-uns, entre autres Esox, Coregonus, Perca, Lota, etc., la connaissance plus approfondie 
de la littérature et l'apparition de nouveaux ouvrages, me donnèrent la conviction qu'il serait plus utile d'examiner d'abord un seul type, mais plus en détail et en y joignant l'étude de la musculature, laissée généralement de côté par les nombreux auteurs qui ont écrit sur les nerfs des Poissons. Cette restriction du sujet éloignait naturellement la possibilité de toute conclusion générale, mais, dans notre pensée, elle devait faire de ce travail un document plus profitable pour des recherches ultérieures.

Sur les six exemplaires de Silure employés, l'un a été préparé frais, deux avaient été conservés dans la formaldéhyde, les trois autres ont été traités par l'acide nitrique. Cette dernière méthode a donné de bons résultats et a permis de poursuivre des ramifications très fines. Appliquée à des têtes entières, elle cause souvent des gonflements considérables qui amènent des déformations et des dislocations très fâcheuses. On atténue ou l'on supprime même cet inconvénient en laissant séjourner les pièces douze à vingt-quatre heures, suivant leur grosseur, dans une solution de formol à $0,5 \%$; on lave ensuite à l'eau courante pendant un temps égal, puis on fait agir l'acide d'abord très dilué $(4 \%)$ et on augmente de jour en jour de $2 \%$ jusqu'à la dose de 12 à $16 \%$. J'ai obtenu ainsi des têtes de Brochets, de Truites et de Lottes sans aucune déformation.

\section{MUSCULATURE DE LA TÊTE}

Il existe peu de travaux concernant la musculature de la tête des Poissons osseux ou, tout au moins, de travaux qui traitent de l'ensemble de cette musculature. En outre, les auteurs qui ont étudié le système nerveux ont très rarement donné des indications sur la musculature, ou ces indications sont tout à fait isolées et peu comparables. L'exposé des muscles de la tête du Silure a donc sa place ici, des renseignements sur ceux-ci étant 
d'ailleurs indispensables pour parler avec quelque exactitude du trajet et surtout de la distribution des nerfs craniens. Les choses déjà connues ne seront brièvement rappelées que pour rendre plus facile la lecture de ce travail et permettre la comparaison avec les indications de la littérature; pour les détails on devra recourir aux mémoires et aux traités généraux cités dans l'index bibliographique.

A ma connaissance, l'ouvrage le plus important sur la musculature céphalique des Poissons est celui de B. VETTER (Untersuchungen zur vergleichenden Anatomie der Kiemen-und Kiefermuskulatur der Fische. Jenaische Zeitschr. 1874 et 1878), dont les recherches ont porté sur Esox lucius, Perca fluviatilis, Cyprinus carpio et Barbus vulgaris. Je suivrai autant que possible l'ordre et la nomenclature adoptés par cet auteur.

On peut distinguer les six catégories suivantes:

I. Muscles oculaires.

II. Muscles de la mâchoire et du barbillon maxillaire.

III. Muscles des extrémités dorsales des arcs viscéraux.

IV. Muscles des extrémités ventrales des arcs viscéraux.

V. Musculature longitudinale ventrale.

VI. Musculature céphalo-scapulaire.

\section{Muscles oculaires}

L'œil du Silure est excessivement petit relativement au volume de la tête, aussi les muscles qui le meuvent sont-ils très faibles. Il y en a quatre droits et deux obliques.

Les quatre muscles, droit supérieur (fig. $6, D s$ ), droit inférieur, droit interne $(D i)$ et droit externe $(D e)$ sont minces et très allongés. Leur origine se trouve sur la face externe de l'alisphénoïde $(\mathrm{Al})^{1}$ autour du trou de l'optique, qui est situé

${ }^{1}$ Sauf indication spéciale, les désignations d'os se rapportent aux figures 1 et 2 (Pl.1). 
à la partie antérieure de cet os. Ils commencent par quatre bandelettes tendineuses translucides, formant une gaîne autour du nerf optique, déjà muni d'une enveloppe résistante propre depuis sa sortie du crâne. Ils ne deviennent plus épais qu'un peu avant la moitié de la distance entre leur origine et l'œil, c'est-à-dire que c'est à partir de là que commence la partie contractile, laquelle reste peu considérable et dépasse de peu le volume du nerf optique. Ce n'est qu'à leur insertion sur le pourtour du globe oculaire que leurs extrémités antérieures sont un peu élargies.

Les deux muscles obliques supérieur et inférieur (fig. 5, 6, $\mathrm{Os}, \mathrm{Oi}$ ) sont beaucoup moins longs que les droits; ils naissent au-dessous de l'élévateur palatin sur la face inférieure du préfrontal (Prf), près de son bord latéral ou, plus exactement, sur la face postérieure de la saillie antéro-latérale de cet os, sur laquelle vient se fixer la pièce antérieure des sous-orbitaux (So). Ils ont la forme de petits cônes allongés, réunis par leur sommet, soit par leur origine qui ne montre pas de portion tendineuse; ils sont dirigés obliquement en arrière, latéralement et en bas et ne s'écartent l'un de l'autre que pour s'attacher sur l'œil, l'un au-dessus, l'autre au-dessous de l'insertion du droit interne.

L'innervation des muscles oculaires a lieu par un oculomoteur commun, un trochléaire qui semble être un rameau de l'oculo-moteur commun, mais ne lui est accolé que temporairement et par un abducteur (voir p. 47 à 50).

II. Muscles DE La vachoIRE ét DU BaRbILlon MaXillaire

A. Adducteur mandibulaire (Pl. 3, fig. 5, 6, Am).

C'est une masse musculaire très puissante, étendue entre l'angle de la mâchoire et le bord antérieur de l'opercule, limitée en haut par le bord latéral du post-frontal (Psf) et du frontal 
principal (Fp), par la pièce postérieure de l'anneau orbitaire, en bas par le préopercule (Pop) et le carré (Qd). Il est divisé en deux portions superposées.

La portion superficielle (fig. 5, côté gauche, $A m_{1}$ ) a son origine sur la face externe du hyomandibulaire $(\mathrm{Hm})$ dans la région postérieure et inférieure, sur le préopercule, sur la partie inférieure du carré, sur la partie latérale de la face inférieure du post-frontal (Psf) et du squameux ( $\mathrm{Sq}$ ). En arrière, la portion superficielle ne forme bien qu'un seul ventre, mais, en avant, elle montre une division en une partie médiale et une partie latérale qui se terminent chacune par une masse tendineuse distincte. C'est entre ces deux tendons que passe la branche mandibulaire interne du nerf maxillaire inférieur. La partie latérale $(a)$ du muscle s'insère, par son tendon qui est très court $\left(a_{t}\right)$, sur le bord supérieur de l'articulaire (Art), à une petite distance de l'articulation de cet os avec le carré, et plutôt sur sa face médiale. En outre, le fascia $\left(f A m_{1}\right)$ qui la recouvre du côté externe, s'étale en bas et en avant sur la face externe de l'articulaire et se prolonge en haut sous forme d'une bande étroite qui se fixe à la base du maxillaire $\left(M_{x}\right)$, réduit à un petit os servant de pièce basale au barbillon. Le tendon de la partie médiale (fig. 5 , côté droit, $b_{t}$ ), descend obliquement en avant, contre la face interne de l'articulaire dont il est séparé par la branche mandibulaire interne du trijumeau, et se fusionne avec le prolongement de la portion profonde qui s'insère sur le cartilage de MEckeL (Pl. 3, fig. 9, C. Mk) et sur le dental (Dt). La portion superficielle ne devient pas tendineuse jusqu'à sa face externe; ses deux tendons apparaissent dans l'intérieur du muscle, ils ne sont visibles que de la face interne ou sur une section.

La portion profonde de l'adducteur mandibulaire (Pl. 3, fig. 5, 6, Am ${ }_{2}$ ) prend naissance à la face externe du hyoman. dibulaire, sur la partie antérieure de cet os, laquelle est sé- 
parée de la partie postérieure, plus considérable, par une crête osseuse (CHm, Pl. 1, fig. 2) dirigée en avant et produite très probablement par ossification de l'origine du fascia de la portion profonde. Une plus faible partie de ses fibres prend naissance sur la région supérieure et postérieure du carré. La face interne du muscle repose sur le métaptérygoïde (Mpt) et sur le carré; la face externe est séparée de la portion superficielle $\left(A m_{1}\right)$ par les muscles élévateur palatin et dilatateur operculaire. Le bord inférieur (latéral) est longé par la branche mandibulaire externe du facial (fig. 5,6, mde); le bord supérieur par le nerf maxillaire inférieur qui le sépare en avant de l'origine du muscle rétracteur du barbillon. Les fibres musculaires sont dirigées, comme celles de la portion superficielle, de haut en bas, d'arrière en avant et de dedans en dehors, et convergent vers l'angle de la bouche. En arrière de ce point, le muscle montre déjà une bande moyenne devenue tendineuse $\left(A m_{2} t\right)$. La partie du muscle située latéralement par rapport à cette bande moyenne, s'insère entièrement sur l'articulaire, de la façon suivante : les fibres musculaires du bord latéral se fixent sans former de tendon distinct sur le bord supérieur de l'articulaire, entre son articulation avec le carré et l'insertion de la partie latérale de la portion superficielle $\left(A m_{1}\right)$, tandis que le reste se prolonge en un tendon $\left(c_{t}\right)$ qui longe la face interne de l'articulaire et s'insère en avant sur celle-ci. La partie médiale du muscle, laquelle possède presque entièrement la bande moyenne tendineuse, s'unit au tendon formé par la partie médiale de la portion superficielle. Il en résulte la formation d'une sorte de lame tendineuse $\left(b_{t}+d_{t}\right.$, fig. 6,9) dirigée obliquement en avant et en bas et appliquée contre la face interne de l'articulaire. La partie inférieure (postérieure) de cette lame se détache bientôt pour constituer un tendon qui s'attache à l'extrémité postérieure du cartilage de MECKel (fig. 9, CMk); le reste, la plus forte partie, va plus en avant et redevient presque complètement musculeux en arri- 
vant dans la rigole du dental (Dt) qui loge le cartilage de MECKEL. Ce prolongement musculaire (fig. 9, $A m_{p}$ ) s'étend sur le bord supérieur de ce dernier, à peu près jusqu'au milieu de sa longueur et s'insère ainsi sur lui jusqu'à la face interne du dental.

Le muscle adducteur mandibulaire est innervé par un fort rameau qui part du commencement du tronc maxillaire commun, traverse d'abord la portion profonde en lui donnant quelques filets, en sort devant le bord antérieur de l'élévateur palatin et se divise. Plusieurs de ses ramifications se replient en arrière, au-dessus de ce dernier muscle, les autres continuent à aller en avant et en bas, toutes pénètrent dans la portion superficielle de l'adducteur mandibulaire. Il serait très possible que, lors de son passage sur la face interne du dental, la branche mandibulaire interne du trijumeau émît des filets destinés au prolongement de l'adducteur mandibulaire inséré en grande partie sur le cartilage de MEckeL, mais, malgré une recherche minutieuse, je n'ai pu m'assurer de leur existence. C'est pourtant dans cette région que le rameau alvéolaire $\left(\mathrm{ra}_{2}\right)$ prend naissance, mais ce dernier court à la surface du muscle et n'a pas d'autres relations avec lui. Il semble, néanmoins, très probable que la branche ou son rameau livre des filets au prolongement musculaire.

L'action de ce muscle est d'attirer la mandibule contre la mâchoire supérieure.

VeTter (48) a trouvé, chez les quatre Téléostéens qu'il a étudiés, des états assez différents pour le muscle appelé souvent masseter. Il l'a toujours vu différencié en deux, trois et même quatre portions, et a regardé la portion la plus profonde, insérée sur le cartilage de MEcKEL, comme étant la plus ancienne et la seule correspondant exactement à l'adducteur des Sélaciens. Le Brochet offrait, parmi les états décrits par VETTER, un état moins différencié. Il semble que le Silure se rapproche, sous 
ce rapport, du Brochet et qu'il lui soit même inférieur en ce que la portion superficielle a la plus forte partie de sa masse tendineuse encore fusionnée avec celle de la portion profonde qui se fixe sur le cartilage de MEckel. La division très nette des deux ventres est probablement due à l'intercalation de l'élévateur de l'arc palatin.

\section{B. Muscles du barbillon maxillaire.}

Le barbillon est formé d'un axe cartilagineux recouvert par la peau et fixé sur le petit os qui représente un os maxillaire rudimentaire. Ses mouvements sont variés; au repos, il est ordinairement dirigé en arrière, parallèlement et à une petite distance du bord latéral de la tête. L'animal peut l'amener en avant par un mouvement horizontal très rapide et le laisse quelquefois assez longtemps dans cette position. Enfin, il peut s'en servir également pour explorer aussi bien au-dessus de sa tête que latéralement, en arrière ou en avant; il l'agite alors comme un fouet.

Les deux paires de barbillons de la face ventrale, désignés par H.-B. Pollard (95) comme tentacules mentonniers et submandibulaires, sont toujours courts et ne possèdent pas de muscles spéciaux. La petite pièce cartilagineuse qui leur sert de base est attachée au fascia du muscle génio-hyoïdien, et ce sont les fibres environnantes de ce muscle qui agissent pour déterminer le petit tremblement caractéristique de ces appendices tactiles qui se produit lorsque le poisson circule. Les nerfs sensibles de ces barbillons dépendent du nerf mandibulaire interne du trijumeau, de même que les quelques filets moteurs qui pénètrent autour de leur base dans le muscle génio-hyoïdien.

Les mouvements variés du tentacule maxillaire (appelé par H.-B. Pollard tentacule maxillo-coronoïdal) sont produits par 
un groupe de trois muscles dirigés presque longitudinalement. Ceux-ci sont aidés par des ligaments dont le plus important (fig. 5, Lmx), cité par Jobert (36), va du bord antérieur de la partie proximale du barbillon, à l'extrémité latérale de l'ethmoïde et à la partie voisine du prémaxillaire. Un autre ligament, beaucoup moins fort, part de l'os maxillaire et va médialement et en arrière s'attacher au palatin; enfin, il y a encore la faible bande aponévrotique fixée latéralement au maxillaire et venant de l'adducteur mandibulaire. On trouvera, dans l'ouvrage de JoBERT (p. 68-75), des renseignements sur l'articulation du barbillon avec sa pièce basale, sur sa musculature et le mécanisme de ses mouvements. Les indications qui viennent d'être données ne font que compléter ces renseignements. Je me bornerai maintenant à indiquer la situation et l'innervation des muscles tentaculaires que j'ai trouvés au nombre de trois ', deux extenseurs et un rétracteur.

Extenseurs supérieur et inférieur (Pl. 3, fig. 5, 6, Esb, Eib). Ces deux muscles tendent à amener le barbillon d'arrière en avant; ils s'étendent de la paroi latérale du crâne jusqu'à la pièce basale du barbillon, presque horizontalement, et médiolatéralement d'arrière en avant.

L'extenseur supérieur est le moins long et le moins épais; c'est un muscle en forme de cône mince, dont la base est tixée sur la partie postérieure de l'orbito-sphénoïde (Ors), un peu en avant et au-dessus du trou de l'optique situé près de la limite entre l'orbito-sphénoïde et l'alisphénoïde $(\mathrm{Al})$. Son origine $\left(E s b_{o}\right)$ est recouverte par la partie antérieure du dilatateur operculaire $\left(D_{o_{a}}\right)$; il n'est séparé de ce muscle que par une petite masse adipeuse renfermant les deux rameaux (ri, rs) de la branche ophtalmique superficielle. En avant, il passe sous l'angle

1 Jobert parle de quatre muscles tentaculaires, mais pour Silurus glanis (pl. III, fig. 32), il n'en figure que trois: un rétracteur, un pretracteur et un adducteur. 
antéro-latéral du préfrontal (Prf) et s'insère par un tendon étroit sur une petite apophyse du maxillaire, dirigée en haut et latéralement.

L'extenseur inférieur est plus long et plus large que le précédent. Il a la forme d'une lame triangulaire dont la longue base est attachée à la paroi cranienne; le long côté postérieur (latéral) est longé par les branches maxillaire supérieure et ophtalmique profonde (mxs, opr), tandis que le côté antérieur est très court et presque perpendiculaire à l'axe longitudinal du corps. Son épaisseur est peu considérable; elle est plus forte vers la base et le côté postérieur du triangle. Son origine (Eibo) commence au niveau et immédiatement devant l'orifice de l'optique et se prolonge en descendant faiblement sur la partie inférieure de l'orbito-sphénoïde, puis en avant sur la partie latérale du palatin (fig. 6, Pal), toujours à une petite distance du bord latéral du parasphénoïde. L'origine de ce muscle est limitée médialement par celle de l'adducteur palatin, et c'est latéralement par rapport à cette limite que passe le nerf sphéno-palatin (sp) avant d'arriver dans le vomer. Les fibres ont la même direction à peu près que celles de l'extenseur supérieur, leur trajet est seulement plus horizontal; elles se prolongent en avant en une bande tendineuse qui s'insère sur la partie supérieure et inférieure du maxillaire et dont le bord latéral vient se confondre avec le tendon du muscle supérieur, près de son insertion.

Rétracteur du barbillon. Il est situé plus en dehors et dans un plan un peu inférieur aux précédents; il est moins long, mais plus épais que ceux-ci. Il est dirigé d'arrière en avant, presque horizontalement et tend à s'élever faiblement du côté du barbillon. Sa forme est celle d'un triangle dont la base serait fixée sur la face externe du métaptérygoïde (Mpt), soit sur la moitié antérieure de cet 0 s. Son bord médial vient toucher le bord latéral de l'extenseur inférieur, et c'est le long de leur ligne de contact supérieure que passent. les branches maxillaire supé- 
RECHERCHES SUR LE SILURUS GLANIS.

rieure et ophtalmique profonde. En arrière, il est croisé dorsalement par l'optique et le nerf maxillaire inférieur, à une petite distance de son origine, qui est recouverte par le bord médial de la portion profonde de l'adducteur mandibulaire. En avant, il devient étroit, mais conserve une forte épaisseur, de sorte qu'il se termine par une lame verticale passant sous le sousorbitaire antérieur, près de son articulation avec le préfrontal et s'insère, par un court tendon, sur le bord postérieur du barbillon, non loin de sa base.

Les deux extenseurs sont innervés par un rameau du tronc hyö̈deo-mandibulaire qui actionne aussi l'adducteur palatin (v. p. 82). Le muscle rétracteur reçoit un nerf venant du tronc maxillaire commun par la voie du rameau destiné à l'adducteur mandibulaire (v.p. 69).

Stannius $(16$, p. 74$)$ admet que les muscles extenseur et rétracteur sont innervés par deux rameaux distincts venant du tronc maxillaire commun; il doit y avoir une inexactitude dans ces observations, comme il y en a une dans son indication d'un seul extenseur.

Bien que l'innervation, telle qu'elle a été mentionnée ici et telle qu'elle sera décrite plus loin (p. 69 et 82), ait été constatée d'une manière absolument certaine, il ne peut être émis d'opinion définitive sur la provenance de la musculature du barbillon maxillaire. En effet, d'après les branches qui livrent les nerfs de ces muscles, les deux extenseurs semblent appartenir au domaine du « facial » et auraient alors la même origine que l'adducteur de l'arc palatin, c'est-à-dire qu'ils proviendraient de la musculature de l'arc hyoïdien, tandis que le rétracteur semble appartenir à l'arc précédent comme l'adducteur mandibulaire. Mais, il se peut aussi que le rameau du muscle rétracteur soit formé d'éléments du facial joints au tronc maxillaire et dont une partie passerait dans le rameau de l'adducteur mandibulaire; dans ce cas, toute la muscula- 
ture du barbillon serait parente de l'adducteur palatin. Une alternative opposée est encore possible, quoique beaucoup moins probable : le nerf des muscles extenseurs, qui semble sortir du facial, dépendrait en réalité du trijumeau, et alors les trois muscles seraient de même origine que l'adducteur mandibulaire. Cependant, des caractères tirés de la situation des muscles rendent ces deux dernières alternatives très peu probables. En effet, les deux extenseurs ont leur origine en connexion avec celle de l'adducteur palatin; la divergence de direction est peu considérable et n'a, du reste, pas grande importance. Pour le rétracteur, sa position et son origine montrent avec une plus grande évidence sa parenté avec l'adducteur mandibulaire. Enfin, l'hypothèse de la parenté des deux extenseurs avec la musculature de l'arc hyoïdien et du rétracteur avec la musculature de l'arc maxillaire est encore appuyée par le fait que le nerf destiné aux deux premiers est une ramification du rameau de l'adducteur palatin, et que le nerf destiné au dernier est une ramification du rameau adducteur mandibulaire.

\section{Muscles des extrémités DoRsales des}

\section{ARCS VISCÉRAUX}

A. Muscles des arcs maxillaire et hyö̈dien.

1. Elévateur de l'arc palatin.

2. Adducteur de l'arc palatin.

3. Adducteur hyomandibulaire.

4. Muscles de l'opercule : dilatateur, élévateur, adducteur.

\section{B. Muscles des arcs branchiaux.}

5. Élévateurs.

6. Obliques dorsaux.

7. Tranverse dorsal.

8. Rétracteur des deux premiers arcs branchiaux.

9. Dilatateur branchial ou rétracteur postérieur. 
A Muscles des arcs maxillaire et hyoüdien.

1. Élévateur de l'arc palatin (Pl. 3, fig. 5, Ep).

Ce muscle est très étalé; il rappelle assez la forme d'un trapèze dont la grande base représenterait l'origine du muscle. Cette origine s'étend sur le préfrontal, le frontal principal et le post-frontal. En arrière, sur ces deux derniers os, elle est resserrée entre l'origine de l'adducteur mandibulaire (portion superficielle) et le bord latéral du dilatateur operculaire (fig. 6, $D o_{p}$ ) et prend la forme d'une lame, en grande partie tendineuse. En avant elle est élargie, charnue; c'est la partie prenant naissance sur la face inférieure du préfrontal et qui n'est pas recouverte par l'adducteur mandibulaire (fig. 5, côté gauche). Ses fibres ont une direction perpendiculaire à celles de ce dernier muscle dont il sépare les deux portions. Il constitue une lame qui est charnue seulement dans sa région moyenne et tendineuse dans la partie antérieure, postérieure et sur toute la surface interne. Ce muscle s'insère sur le hyomandibulaire, suivant une ligne oblique d'avant en arrière et de bas en haut, allant de l'extrémité inférieure de l'arête servant à l'insertion de la portion superficielle de l'adducteur mandibulaire, jusqu'à l'angle antérieur et supérieur de l'opercule, en passant sur le tronc hyoïdeo-mandibulaire au moment où il vient de sortir de l'os hyomandibulaire et se bifurque en branches mandibulaire et hyoïdienne.

Ce muscle soulève le hyomandibulaire en le tirant en avant, ce qui revient à écarter la partie inférieure de l'arcade ptérygo-palatine de la ligne médiane.

L'innervation a lieu au moyen d'un rameau venant du tronc maxillaire commun et qui pourvoit aussi le dilatateur operculaire (voir p. 69). 
Ce muscle ne semble pas présenter d'autre particularité que d'être inséré exclusivement sur le hyomandibulaire, bien que son origine paraisse être beaucoup plus étendue que chez les Téléostéens examinés par VETTER.

\section{Adducteur de l'arc palatin.}

L'origine de ce muscle s'étend sur le bord latéral du long parasphénoïde (Ps), de la limite entre l'os pétreux et l'alisphénoïde (Al) jusqu'à son extrémité antérieure. Elle atteint donc une grande longueur, mais, par contre, son épaisseur est très faible; elle est longée supérieurement par le nerf sphénopalatin. Les fibres ont une direction transversale, un peu inclinée d'avant en arrière et de haut en bas. Le muscle devient plus épais dans sa région latérale, par laquelle il s'insère sur la face interne du métaptérygoïde en avant, et du hyomandibulaire en arrière, sans former de tendon. En avant, il est limité par la partie latérale du vomer, en arrière par les élévateurs branchiaux dont il est séparé par le tronc hyoïdeo-mandibulaire qui sort du crâne et va traverser plus bas le hyomandibulaire; inférieurement il est recouvert par la muqueuse du plafond de la cavité buccale. Sa partie médiale antérieure se trouve sous l'extenseur inférieur du barbillon et sa partie médiale postérieure sous la branche maxillaire inférieure. La contraction de ce muscle produit donc le rapprochement de l'arc ptérygo-palatin vers la ligne médiane.

Il est innervé par un rameau venant du tronc hyoïdeo-mandibulaire, destiné également aux deux extenseurs du barbillon maxillaire (voir p. 82).

On voit que, d'après la situation respective des deux extenseurs du barbillon et de l'adducteur palatin, il n'y a pas de difficulté à admettre la communauté d'origine de ces muscles, telle qu'elle a été exposée précédemment (p . 11). 
3. Adducteur hyomandibulaire (Pl. 3, fig. 5, Ahm).

L'adducteur hyomandibulaire du Silure est un muscle très peu considérable qui présente plusieurs particularités intéressantes par rapport à celui des autres Téléostéens. En effet, d'après VETTER, son origine se trouverait généralement sur l'os pétreux (chez les Cyprins, sur le pétreux et une partie du parasphénoïde). Chez le Silure, elle se trouve complètement sur la face inférieure du squameux $(\mathrm{Sq})$, médialement par rapport à celle de l'élévateur operculaire qui occupe le bord latéral de cet os; elle est repoussée en arrière probablement par les élévateurs branchiaux qui prennent ici une extension particulière. Aussi, le muscle n'est-il pas du tout situé sous le hyomandibulaire, mais immédiatement en arrière de cet os. Il est de forme triangulaire (plus exactement trapézoïde). Son sommet est fixé sur le processus operculaire du hyomandibulaire et son bord antérieur sur le bord postérieur du même os; il est limité en arrière par l'adducteur operculaire et sa face médiale est recouverte par la muqueuse de la cavité branchiale. Il pourrait facilement être pris pour un muscle de l'opercule, mais son insertion sur la face interne du hyomandibulaire ne laisse aucun doute sur sa fonction.

Ce muscle est innervé par un rameau du tronc hyoïdeo-mandibulaire allant aussi dans l'élévateur et l'adducteur de l'opercule.

\section{Muscles de l'opercule.}

Dilatateur operculaire ( $\mathrm{Pl}$. 3, fig. 5, 6, Do). Il est complètement distinct de l'élévateur palatin, bien que sa région postérieure et latérale située immédiatement derrière ce muscle paraisse au premier abord en faire partie. Cette région postérieure est la seule visible sur la figure $5\left(D o_{p}\right)$; mais si on enlève cette sorte de grand avant-toit formé par le frontal en 
dehors de la paroi latérale de la cavité cranienne, on découvre une région antérieure beaucoup plus étendue que l'autre et dont l'origine se trouve sur la face inférieure du frontal principal, entre sa suture avec l'orbito-sphénoïde et l'origine de l'élévateur palatin (fig. $6, D_{o_{a}}$ ). En arrière, l'origine se continue sur la saillie d'articulation du post-frontal avec le hyomandibulaire, puis sur la face inférieure du petit avant-toit formé par le squameux au-dessus de son articulation avec le hyomandibulaire. La région postérieure est une lame peu épaisse, la région antérieure est un peu plus forte. Cette dernière a ses fibres fortement dirigées en arrière, et son bord antérieur devient bientôt tendineux. En arrière, elle est en parfaite continuité avec la région postérieure. Toutes les fibres du muscle convergent vers la tête d'articulation de l'opercule, où l'insertion a lieu par un tendon en forme de ruban.

Le dilatateur tend à tirer l'opercule en avant et en haut, ce qui revient à faire tourner ce dernier et le subopercule comme un battant de porte autour du préopercule et, par conséquent, à ouvrir la fente operculaire.

Il recoit une ou deux ramifications d'un rameau sortant du tronc maxillaire commun et se rendant à l'élévateur palatin (voir p. 69).

Élévateur operculaire (Pl. 3, fig. 5, Eo). Les fibres de ce muscle ont une direction presque verticale. L'origine se trouve en haut sur la face inférieure, au bord latéral du squameux, sur la partie comprise entre le hyomandibulaire et le supra-claviculaire (Sc). Par sa face interne, il repose sur les adducteurs hyomandibulaire et operculaire; sa face externe est recouverte par la peau, et il s'insère en bas sur le bord supérieur de l'opercule (Op).

Il agit simultanément avec le suivant pour produire l'occlusion de la fente operculaire.

Adducteur operculaire (Pl. 3, fig. 5, Ao). Il prend aussi 
naissance sur la face inférieure du squameux, médialement par rapport à l'élévateur. En avant, il est limité par l'adducteur hyomandibulaire qui recouvre même un peu sa partie antérieure; sa face médiale est recouverte par la muqueuse de la cavité branchiale. Il s'insère sur la face interne de l'opercule, dans la région supérieure et postérieure de cet 0 s.

L'innervation se fait par un filet d'un rameau du tronc hyoïdeomandibulaire qui actionne aussi l'adducteur hyomandibulaire et l'élévateur operculaire. Le plus souvent, les Téléostéens posséderaient, semble-t-il, d'après VetTer (48) et GoRonowitsch (100), un rameau commun pour les deux muscles operculaires, et un autre pour l'adducteur hyomandibulaire. Chez le Silure, un état plus simple s'est conservé par le fait que le dernier muscle est assez loin en arrière du tronc hyoïdeo-mandibulaire, et qu'il reste en contact avec les deux muscles operculaires.

\section{B. Muscles des arcs branchiaux.}

La musculature des arcs branchiaux présente des différences assez considérables avec celle des autres Poissons osseux et c'est surtout à cause de celà qu'il m'a paru nécessaire de faire précéder ce travail sur les nerfs craniens d'indications précises sur les muscles de la tête.

Chacun des quatre arcs branchiaux, depuis le coude jusqu'à l'extrémité dorsale, est formé de deux pièces, l'épibranchial et le pharyngo-branchial (Pl. 3, fig. 7 et 8, Ebr I-IV, Pbr I-IV). Dans les deux premiers arcs, les deux pièces sont à peu près de même longueur et ne présentent rien de particulier, si ce n'est que les deux pharyngo-branchiaux sont appliqués l'un contre l'autre et se soudent médialement en une pièce commune (Pbr I+II). Dans les deux arcs postérieurs, les épibranchiaux s'infléchissent plus fortement vers la ligne médiane que ceux des arcs précédents, aussi existe-t-il entre l'épibranchial III et le pha- 
ryngo-branchial II un espace assez grand, qui va en augmentant dans la direction latéro-médiale. Près de leur extrémité proximale, ils possèdent chacun une apophyse, dont l'une, la postérieure, est plus grande et entre en contact avec l'autre.

Les pharyngo-branchiaux III et IV sont deux pièces courtes, entièrement ou en partie cartilagineuses. Ils présentent une disposition particulière : le dernier est dirigé obliquement d'arrière en avant et de dehors en dedans, l'épibranchial IV s'appuie contre son extrémité postérieure et l'épibranchial III contre son bord latéral, près de son extrémité antérieure; enfin le pha. ryngo-branchial III n'est plus en relation directe avec l'épibranchial III, il est devenu à peu près parallèle à l'axe du corps et relie l'extrémité commune des pharyngo-branchiaux I et II avec l'extrémité antérieure du pharyngo-branchial IV.

La plaque pharyngienne munie de dents (fig. 8, Pph, supposée transparente) est simplement appliquée contre la face inférieure des deux derniers arcs, dont elle recouvre l'extrémité des épibranchiaux, la partie postérieure du pharyngo-branchial III et le pharyngo-branchial IV.

\section{5. Élévateurs branchiaux (Pl. 3, fig. 7, Elb ${ }_{1-4}$ ).}

Ils offrent, chez le Silure, un état assez différent de celui décrit pour quelques autres Poissons par différents auteurs. Ils sont au nombre de quatre, un pour chaque arc. Leurs origines sont distinctes, mais rapprochées les unes des autres sur le pétreux, au-dessous de l'articulation du hyomandibulaire.

Les deux premiers ont leur origine l'une à côté de l'autre, de telle façon que le second est situé médialement par rapport au premier. Ils sont formés chacun d'un seul faisceau aplati, dirigé en bas, en arrière et un peu latéralement; leur extrémité inférieure, plus large, s'insère, respectivement à l'extrémité médiale des épibranchiaux I et II, sur une faible saillie du bord dorsal postérieur. 
Les élévateurs des deux derniers ares sont plus forts et montrent une disposition particulière. L'origine du troisième (fig. $\left.7, E l b_{3}\right)$ est située derrière celle du quatrième $\left(E l b_{4}\right)$. Les deux muscles sont dirigés en arrière, en bas et plutôt médialement et descendent dans l'espace entre le pharyngo-branchial II et l'épibranchial III. Ils se croisent comme les deux branches d'un $X$ et de telle façon que l'élévateur du quatrième arc passe latéralement par rapport à l'élévateur du troisième arc (fig. 8). Ce dernier muscle, toujours dirigé en arrière, s'insère sur l'extrémité médiale de l'épibranchial III, à son bord antérieur. Enfin le quatrième muscle (fig. 8, Elb ), le plus long, croise inférieurement l'épibranchial III; il passe donc entre cet os et la plaque pharyngienne dentée et vient s'attacher sur l'extrémité médiale de l'épibranchial IV, à son bord antérieur. L'insertion de ces deux élévateurs postérieurs se trouve donc sur la face inférieure des pièces, en grande partie cartilagineuses, servant d'appui à la plaque pharyngienne dentée (Pph). De plus, une bonne partie des fibres situées du côté ventral de ces deux muscles se fixe sur la face supérieure de la plaque pharyngienne dentée, soit sur une partie de sa moitié latérale. En résumé, l'extrémité inférieure des deux muscles est engagée entre l'extrémité des épibranchiaux III et IV d'une part et la plaque pharyngienne d'autre part; ils s'insèrent principalement en haut et en arrière sur les deux premières pièces, puis en bas, et sans doute secondairement, sur la dernière pièce.

Ces muscles sont innervés par les rameaux branchiaux du glosso-pharyngien et du vague. Le glosso-pharyngien, au moment où il passe derrière le muscle antérieur, lui envoie un filet. Le premier tronc branchial livre un filet pour le second muscle lorsqu'il est parvenu près du bord médial de son insertion. Il se divise ensuite en un rameau passant devant le muscle (r. postérieur du premier arc) et un autre, plus fort, passant derrière (r. antérieur du deuxième arc). L'alternance entre les deux 
muscles postérieurs et les autres rameaux branchiaux du vague n'existe plus; le rameau antérieur du deuxième tronc, qui devrait, dans la règle, passer devant le troisième élévateur, passe en arrière des deux muscles. D'après ceci, on pourrait penser que les deux élévateurs branchiaux postérieurs ne sont que des parties du deuxième muscle, qui se sont séparées de celui-ci et se sont insérées secondairement sur les troisième et quatrième arcs. Mais le mode d'innervation ne permet pas d'admettre cette opinion, les deux muscles étant actionnés par le deuxième et le troisième tronc branchial. L'élévateur du troisième arc reçoit très probablement un filet de la branche antérieure du deuxième tronc lorsque celle-ci passe à une petite distance de son bord postérieur; j'ai du moins observé la naissance d'un filet qui prenait cette direction, mais je n'ai pas vu s'il entrait réellement dans le muscle. Quant à l'innervation de l'élévateur du quatrième arc, elle a été constatée plus exactement. Elle a lieu par un filet qui sort de la branche antérieure du troisième tronc branchial, descend entre l'extrémité médiale des deux derniers épibranchiaux et pénètre dans l'extrémité inférieure du muscle.

En résumé, on aurait donc chez le Silure quatre élévateurs branchiaux qui appartiendraient, non-seulement par leur insertion, mais aussi par leur provenance, à chacun des arcs, l'innervation devant être regardée comme un caractère plus important que le trajet des nerfs par rapport aux muscles. Cependant, tout en admettant que les rapports de situation exposés plus haut n'ont qu'une importance secondaire, il faut reconnaitre qu'ils sont inexplicables pour le moment.

Maintenant, il faut ajouter que le Silure ne possède pas d'autres élévateurs que les quatre qui viennent d'être décrits, tandis que les autres Téléostéens ont ordinairement, autant que nous le savons, des élévateurs externes, des élévateurs internes et quelquefois des élévateurs postérieurs (CUVIER, VETTER, VoGT et 
Yung, etc.) Mais VetTer (48, p. 533) a montré que dans ces cas, les externes seuls ont conservé leur situation primitive et que les autres en sont dérivés secondairement. Le Silure présenterait donc un état moins différencié, qu'il faut peut-être rapprocher de l'état trouvé par C. BRUch (p. 11) chez le Saumon. Ceci ne signifie cependant pas que les élévateurs branchiaux du Silure soient restés dans leur situation primitive, ce qui n'est vrai que pour les deux premiers. Les deux suivants ont éprouvé des modifications notables qui pourraient les faire passer facilement pour des muscles d'une autre provenance. Leur extension sur la face inférieure de l'extrémité dorsale des arcs et le déplacement de leurs origines montrent à la fois une adaptation des deux muscles afin d'élever et de tirer plus facilement en avant la pièce pharyngienne dentée, et une spécialisation du quatrième muscle comme contracteur branchial. Le rôle des deux muscles postérieurs n'est donc plus simplement d'élever les arcs, mais aussi de les rapprocher les uns des autres et de plus, de faciliter l'acte de la déglutition; en conséquence, ils sont plus forts que les deux antérieurs.

6. Obliques dorsaux (Pl. 3, fig. 7, 8, Od).

Il paraît exister, parmi les Téléostéens, des différences nombreuses dans la disposition de ces muscles. VETTER (p. 508-510) en a trouvé entre les Cyprins, le Brochet et la Perche. Le Silure semble offrir un état intermédiaire entre celui du Brochet et celui de la Perche.

Les obliques dorsaux du Silure constituent une masse musculaire assez épaisse, étendue sur la face supérieure de l'extrémité dorsale des arcs. Ils comprennent deux couches dont l'antérieure $\left(O d_{a}\right)$ recouvre presque complètement la postérieure $\left(O d_{p}\right)$. La première prend naissance sur le bord postérieur du premier 
pharyngo-branchial, immédiatement avant la formation de la pièce commune aux deux premiers pharyngo-branchiaux, sur le bord postérieur de cette dernière pièce, puis sur le pharyngobranchial III ; ses fibres sont dirigées latéralement et en arrière et s'insèrent sur l'apophyse de l'épibranchial III et sur la partie voisine de l'apophyse de l'épibranchial IV. Près de son origine, ce premier muscle semble être formé de trois faisceaux. En arrière, il devient fusiforme et la séparation des faisceaux ne peut pas être poursuivie, sauf pour celui (fig. 8, OdI) qui prend naissance sur le pharyngo-branchial I, mais dont le sommet est pourtant fusionné avec celui de l'oblique dorsal antérieur. La deuxième couche a son origine sur une petite partie du pharyngobranchial III et sur le pharyngo-branchial IV. Elle s'insère en arrière sur l'apophyse de l'épibranchial IV, mais une partie de ses fibres se perd dans le constricteur du pharynx $(C p h)$.

Ces deux muscles sont innervés par des rameaux des trois troncs branchiaux du vague (v. p. 109). Leur action est de comprimer la cage branchiale d'arrière en avant.

Les obliques dorsaux du Silure montrent donc une disposition qui rappelle parfaitement celle des obliques dorsaux supérieurs du Brochet; quant aux obliques dorsaux inférieurs de ce dernier Poisson, ils manquent complètement, comme chez la Perche. De plus, il existe chez le Silure un muscle (fig. 7,8 , $M p h i)$ excessivement court reliant le coude du quatrième arc, ou plus exactement l'extrémité supérieure du cérato-branchialIV,à l'extrémité postérieure libre de l'os pharyngien inférieur (Phi). Ce muscle reçoit un filet de la branche pharyngienne inférieure du vague. VETTER a trouvé un muscle analogue, seulement chez le Brochet $(48$ p. 509) et le rattache aux obliques dorsaux, sous le nom d'oblique dorsal postérieur. Chez le Silure il est aussi en rapport immédiat avec le constricteur pharyngien ( $C p h)$.

Comme il a été dit plus haut, le constricteur pharyngien est également en continuité avec l'oblique dorsal postérieur et il 
faut encore ajouter ici qu'une partie du même muscle tend à se spécialiser comme muscle de l'appareil branchial. Il s'agit d'un contingent de fibres assez considérable occupant l'angle interne du coude du quatrième arc, c'est-à-dire attaché en bas à l'extrémité latérale du cérato-branchial IV et en haut sur l'extrémité latérale de l'épibranchial IV. C'est donc un prolongement du constricteur pharyngien en avant de la charnière du quatrième arc, servant à rapprocher la moitié ventrale de l'arc, de la moitié dorsale et à tirer en même temps l'os pharyngien inférieur, garni de petites dents, contre l'os pharyngien supérieur. Il est désigné dans les figures 7 et 8 comme constricteur du quatrième arc (Cons IV), de même que la partie du constricteur pharyngien dont il est le prolongement.

\section{Transierse dorsal (fig. 8, $T d$ ).}

C'est une plaque musculaire peu épaisse, étendue à la face inférieure du parasphénoïde et reliant ensemble les ares des deux côtés. Sa surface inférieure est recouverte par la muqueuse de la cavité pharyngienne; il naît donc sur la face interne (inférieure) des extrémités dorsales des arcs. Il est fixé de chaque côté sur la pièce osseuse provenant de l'union des pharyngobranchiaux I et II et sur la face inférieure du pharyngo-branchial III. En arrière, il touche à la musculature pharyngienne, mais s'en distingue nettement par les prolongements latéraux qu'il forme pour se fixer sur les arcs, ainsi que par la direction de ses fibres qui est franchement transversale, tandis que celle des fibres du pharynx tend à être longitudinale et n'est transversale que près de la ligne médiane. L'innervation a lieu par des rameaux des deuxième et troisième trones branchiaux du vague. Ils sortent de la branche antérieure des troncs, non loin de sa naissance, vont en avant en passant à la face infé- 
rieure des obliques dorsaux et pénètrent dans le transverse par sa face supérieure.

VETTER a trouvé, comme muscle commun aux quatre espèces examinées, un transverse reliant les quatrièmes arcs et quelquefois aussi les troisièmes arcs; seule, la Perche avait en outre un muscle antérieur distinct entre les épibranchiaux II. Le Silure présenterait donc un état plus indifférent, puisque les trois arcs antérieurs sont réunis avec ceux de l'autre côté par une seule masse musculaire et que le quatrième arc ne possède pas encore de transverse, mais donne seulement insertion à des fibres du constricteur pharyngien. Ces fibres, dirigées obliquement en avant et médialement, sont situées immédiatement derrière le transverse dorsal et sont innervées par des filets de la branche pharyngienne inférieure.

8. Rétracteur des deux premiers arcs branchiaux ou rétracteur antérieur (fig. 7, Rba).

Je désigne ainsi un muscle qui ne semble avoir de commun avec les rétracteurs observés chez les autres Téléostéens, que la fonction; l'origine, l'insertion et l'innervation en sont complètement différentes.

L'origine se trouve sur la face inférieure de l'exoccipital, près de l'arête inféro-postérieure du crâne, médialement par rapport à l'origine de l'adducteur operculaire, qui est située sur le squameux. C'est un long muscle, ayant la forme d'un ruban, dirigé d'arrière en avant, de haut en bas et de dehors en dedans. Sa face supérieure est tournée médialement et appliquée contre la paroi du crâne, en arrière contre l'exoccipital, en avant contre l'os pétreux où elle est croisée par le glosso-pharyngien, qui va s'infléchir latéralement pour passer entre les élévateurs branchiaux. A sa face inférieure (en même temps latérale) le muscle est croisé en arrière par les troncs branchiaux qui 
viennent de se diviser et le séparent de l'insertion des obliques dorsaux; plus en avant, il repose sur le faisceau antérieur du premier oblique dorsal, passe médialement par rapport aux élévațeurs et s'insère enfin sur le bord supérieur et latéral de la pièce formée par la réunion des extrémités des deux premiers pharyngo-branchiaux (Pbr I + II).

Il est innervé par le glosso-pharyngien (voir p. 102).

Son action consiste à tirer en arrière les deux premiers arcs, mouvement qui est, du reste, transmis aux arcs postérieurs par l'intermédiaire des pharyngo-branchiaux disposés comme il a été dit plus haut. Agissant seul, c'est un antagoniste des élévateurs et surtout des deux élévateurs postérieurs; s'il agit en même temps que ces derniers, les arcs sont alors comprimés les uns contre les autres.

Enfin, j'ajouterai que, par son insertion et par la direction de ses fibres, ce rétracteur de l'appareil branchial fait penser à un oblique dorsal dont l'origine ne se trouverait plus sur les arcs branchiaux, mais se serait déplacée vers le haut et en arrière, et serait parvenue sur le crâne.

9. Dilatateur branchial ou rétracteur postérieur (fig. 7, Rbp).

Ce muscle ne paraît pas non plus pouvoir être homologué aux rétracteurs branchiaux indiqués par les auteurs chez d'autres Téléostéens; cependant, par son insertion, il se rapprocherait de celui de la Perche (Cuvier, 7, p. 303. Vetter, 48, p.511).

L'origine est sur la face ventrale du crâne, dans le voisinage de l'angle postérieur et latéral de celui-ci ou, plus exactement, sur l'arête inférieure de l'exoccipital, près de l'articulation de cet os avec le supra-claviculaire ( $\mathrm{Scl}$ ), un peu en arrière et latéralement par rapport à l'origine du rétracteur antérieur. C'est un muscle moins long, mais plus épais et plus large que le rétracteur antérieur, plus fortement incliné vers le bas. Il 
descend parallèlement à l'arête postérieure et inférieure du crâne, de telle façon que les branches du vague proprement dit arrivent contre sa face médiale et qu'elles sont séparées par lui en deux groupes, les unes allant en avant, les autres le croisant en arrière. Plus bas, il est parallèle au bord postérieur du dernier oblique dorsal et s'insère sur la partie postérieure et médiale du quatrième pharyngo-branchial, par conséquent sur l'une des pièces cartilagineuses servant d'appui à la plaque pharyngienne supérieure.

L'innervation a très probablement lieu par un rameau du troisième tronc branchial (p. 112).

L'action de ce muscle peut s'ajouter à celle du précédent pour tirer l'appareil branchial en arrière, mais son rôle le plus important doit être, quand il agit seul, d'élever et de tirer en arrière l'os pharyngien supérieur; il fonctionne alors comme antagoniste principal des deux élévateurs postérieurs. Enfin, lorsque sa contraction est combinée avec celle des élévateurs, il dilate l'appareil branchial.

Quant à la provenance, on peut également appliquer à ce muscle ce qui a été dit pour le rétracteur antérieur; son insertion et la direction de ses fibres semblent indiquer qu'il est parent des obliques dorsaux et, par conséquent, du constricteur pharyngien, avec lequel ces derniers sont, comme on l'a vu plus haut, en continuité. La direction plus ou moins longitudinale des fibres du rétracteur ne peut pas nous empêcher d'admettre cette opinion. En effet, chez le Silure, la région du constricteur pharyngien avoisinant l'insertion du muscle qui nous occupe a ses fibres dirigées obliquement en arrière, presque parallèlement à l'os pharyngien inférieur, soit dans un sens plus longitudinal que transversal. 


\section{Muscles Des eXtrémités Ventrales DeS} ARCS VISCÉRAUX

A. Muscles des arcs maxillaire et hyö̈dien.

1. Intermandibulaire.

2. Genio-hyoüdien.

3. Hyo-hyoïdien.

B. Muscles des arcs branchiaux et de l'arc pharyngien.

4. Pharyngo-arcuo-hyoïdien.

5. Transverse ventral.

6. Pharyngien transverse.

7. Pharyngo-claviculaires [externe et interne].

\section{A. Muscles des arcs maxillaire et hyoüdien.}

\section{Intermandibulaire (Pl. 3, fig. 9, $\mathrm{Im}$ ).}

Muscle reliant les extrémités antérieures des deux dentaux et fixé sur leur face interne. Il semble être plus fort, toutes proportions gardées, que celui des autres Téléostéens. Ce qui le distingue encore, c'est qu'il n'est pas complètement indépendant; toute sa partie postérieure, la plus considérable, est enchâssée dans le génio-hyoïdien, et la limite entre les deux muscles n'est marquée que par une étroite inscription tendineuse, visible aussi bien sur la face supérieure que sur la face inférieure. Vu ventralement, le muscle a ainsi la forme d'un losange, dont les côtés antérieurs sont formés par l'échancrure des os dentaux et les côtés postérieurs par l'inscription tendineuse. Chez les autres Poissons osseux, le muscle intermandibulaire est séparé complètement du génio-hyoïdien; les insertions de ce dernier ne font que passer au-dessus et au-dessous des siennes. 
L'innervation a lieu par un rameau (fig. 9, rim) de la branche mandibulaire interne du trijumeau; les rameaux du tronc hyoïdeo-mandibulaire (facial) y restent sûrement étrangers (voir p. 77 et 78 ).

\section{Génio-hyoüdien (fig. 9, Gh).}

Il présente également des particularités intéressantes. C'est une masse musculaire considérable remplissant l'espace entre l'arc hyoïdien et la mâchoire inférieure et recouverte inférieurement par la peau. Les muscles des deux côtés viennent se rencontrer médialement en avant.

Le génio-hyoïdien prend naissance sur la région inférieure et postérieure de la face latérale du cérato-hyal (Chy) et sur une petite partie de l'épihyal (Ehy), soit sur la région située en avant de la base des rayons branchiostèges 9 à 14 . A son origine, le muscle est assez épais, mais en avant, il devient en même temps plus mince et plus large; il se réunit sur la ligne médiane avec son congénère de l'autre côté, et cette place est indiquée par une étroite inscription tendineuse, visible sur les deux faces.

Le muscle conserve sa plus grande épaisseur à son bord latéral, par lequel il s'insère en avant, sans former de tendon, contre le bord inférieur du dental. Il s'amincit rapidement dans la direction latéro-médiale et constitue alors une lame mince recouvrant ventralement le muscle hyo-hyoïdien inférieur. Son bord médial est libre et, se continuant avec celui de son congénère, donne lieu à une courbe en forme de cintre. Celle-ci est visible de l'extérieur, parce que la peau se replie autour du bord médial pour former au-dessus du muscle un cul-de-sac assez profond. Ce dernier est relié en avant, par une membrane fibreuse, avec le fond du repli membraneux qui forme un sillon du plancher buccal contre la face interne du dental. 
Le génio-hyoïdien du Silure est innervé par un fort rameau de la branche mandibulaire interne du trijumeau (fig. $9, \mathrm{c}$; voir p. $77,78,89)$.

Voici, d'après Vetter (48, p. 515), l'action de ce muscle : "Lorsque l'extrémité inférieure de l'arc hyoïdien est fixée, soit tirée en arrière par contraction du coraco-hyoïdien, le géniohyoïdien transmet ce mouvement en l'amplifiant à la mâchoire inférieure, il agira donc essentiellement comme rétracteur de celle-ci... Mais si la mâchoire est fixée par l'adducteur mandibulaire, le génio-hyoïdien tirera en avant les extrémités ventrales de l'arc hyoïdien et de tous les ares branchiaux, étendra la langue et élargira la fente branchiale par en bas. »

\section{Hyo-hyoïdien (fig. 9, Hh).}

Ce muscle comprend deux parties bien distinctes, quoique incomplètement indépendantes l'une de l'autre. L'une, le hyohyoïdien supérieur, est le muscle de la membrane branchiostège, l'autre, le hyo-hyoïdien inférieur, ou hyo-hyoïdien proprement dit, offre, chez le Silure, une extension et une situation particulières.

Le hyo-hyö̈dien supérieur (Hhs) a son origine (fig. $5, H h s_{0}$ ) sur la face interne de l'opercule $(O p)$, où elle s'avance assez haut, jusqu'à quelque distance de l'insertion de l'adducteur operculaire, puis sur la région postérieure de l'interopercule (Iop). Il forme alors une couche mince, très étalée, dirigée en avant et en bas sur les rayons branchiostèges. Ses fibres cessent d'être continues, elles sont divisées par ceux-ci en segments, qui s'attachent au bord antérieur d'un rayon et au bord postérieur du précédent. Le muscle est cependant étendu sur toute la largeur de la membrane (fig. 9, Hhs). Du côté médial, il devient un peu plus épais; en avant du premier rayon, les fibres ont une direction longitudinale et constituent un prolongement trian- 
gulaire qui va s'insérer sur la partie antérieure du cérato-hyal, au moyen d'une lame tendineuse formée par sa couche supérieure. Les fibres périphériques, soit la couche inférieure de ce prolongement triangulaire, arrivent contre le hyo-hyoïdien inférieur, à peu près au niveau du tiers antérieur du premier rayon, s'infléchissent médialement et ne peuvent plus être distinguées de ce dernier muscle; elles se réunissent médialement avec celles de l'autre côté par l'intermédiaire d'une mince inscription tendineuse.

Le hyo-hyö̈dien inférieur (Hhi) n'est pas une simple continuation du précédent, comme VETTER l'a indiqué pour d'autres Poissons. Il est beaucoup plus considérable que chez ceux-ci, a une origine parfaitement distincte, et s'étend sur la face inférieure (externe) de l'arc hyoüdien.

Son origine est très étendue; elle commence en arrière sur l'extrémité antérieure et le bord supérieur de l'épihyal, se prolonge en avant, d'une part, sur le bord supérieur du long cérato-hyal, d'autre part, en bas sur la face externe de l'épihyal, le long de la suture de cet os avec le cérato-hyal; elle n'atteint pas la base des rayons branchiostèges postérieurs ', mais en est séparée par l'origine du génio-hyoïdien. Elle se continue donc en avant de cette dernière, sur la partie postérieure du cératohyal, et parvient à la base du neuvième rayon à côté de l'origine du génio-hyoïdien, de laquelle on ne peut la séparer exactement; elle s'étend, plus en avant encore, sur la face inférieure des huit premiers rayons branchiostèges, dont elle recouvre la base et une partie qui va en augmentant des rayons postérieurs vers les antérieurs. Elle atteint enfin le premier rayon dont elle recouvre tout le tiers antérieur.

T'outes les fibres naissant sur le bord supérieur de l'épihyal

1 Les deux derniers rayons sont fixés sur l'épihyal; tous les autres, au nombre de treize, sur les deux tiers postérieurs du cérato-hyal. 
et du cérato-hyal descendent sur la face externe de ces os, mais elles vont moins en avant que ceux-ci et croisent par conséquent leur direction sous un angle aigu. Celles qui naissent sur la face externe de l'arc hyoïdien et sur la base des rayons branchiostèges ont une direction parallèle aux autres; en avant du premier rayon vient s'ajouter à elles le faible contingent des fibres superficielles du hyo-hyoïdien supérieur.

En résumé, tout le bord inférieur (médial) du muscle est attaché soit sur la face externe de l'arc, soit sur la base des rayons branchiostèges, et tout le bord supérieur (latéral) sur le bord supérieur du cérato-hyal, sauf une petite partie antérieure située sur l'hypobranchial et dont nous allons parler bientôt au sujet de l'insertion.

En avant, le muscle devient un peu moins large, mais conserve la même épaisseur; ses fibres prennent une direction transversale et il se réunit alors sur la ligne médiane avec son congénère de l'autre côté. Une inscription tendineuse mince se trouve seulement dans la région postérieure de la ligne de réunion, dans celle où le contingent d'éléments du muscle hyo-hyoïdien supérieur vient se joindre au hyo-hyoïdien inférieur; en avant, il y a passage complet des fibres d'un côté à l'autre.

Pour l'insertion sur des parties osseuses, il y a un tendon rubané $\left(H h i_{t}\right)$, que l'on voit sortir du bord antérieur du muscle. Il se dirige en avant et descend sur le bord antérieur de l'hypohyal inférieur sur lequel il s'attache, très près de la ligne médiane.

Ce tendon ne peut pas être le prolongement de la partie du muscle dont il sort, car la direction des fibres lui est alors presque perpendiculaire. Lorsqu'on coupe ces fibres, on voit que le tendon traverse toute la partie antérieure du muscle et que, en arrière, il forme une lame tendineuse médiane, d'où part aussi le tendon destiné à l'autre côté. La lame tendineuse médiane est horizontale; elle commence à l'intérieur de la masse muscu- 
laire même, sur l'inscription tendineuse dont on vient de parler et qui représente une mince lame verticale, peu élevée.

D'après ce qui a été dit plus haut, il est facile de voir que l'inscription tendineuse est surtout formée par le contingent de fibres du hyo-hyoïdien supérieur qui vient s'adjoindre au hyohyoïdien inférieur et que, par conséquent, la lame tendineuse appartient en partie au muscle hyo-hyoïdien supérieur. Mais il est certain qu'elle dépend aussi du muscle hyo-hyoïdien inférieur, car, dans la masse de celui-ci, dans sa région médiale, apparaissent des filets tendineux qui viennent s'unir à l'inscription tendineuse médiane, en produisanit une sorte de nœud. C'est de ce nœud que part la lame tendineuse médiane, divisée bientôt après en deux tendons, et dont l'insertion a été déjà expliquée.

On peut ajouter qu'il se sépare de chacun de ces tendons un faible cordon médial $\left(H h i_{t}\right)$, qui va s'insérer de l'autre côté, quelquefois au même point que le tendon symétrique, d'autre fois, un peu médialement par rapport à celui-ci. Cette disposition rappelle tout à fait, en petit, celle décrite et figurée par VETTER pour le muscle hyo-hyö̈dien inférieur chez le Brochet $\left(48\right.$, p. 516 , Pl. 3, fig. 10, Hhi ${ }_{1}$ et $\left.\mathrm{Hhi}_{2}\right)$. Enfin, par son aponévrose, le muscle est encore attaché an bord antérieur de l'hypohyal et à la membrane formant le plancher buccal antérieur.

L'innervation des deux muscles a lieu par le nerf hyö̈dien (hy) ou branche postérieure du tronc hyoïdeo-mandibulaire (facial). Le muscle supérieur reçoit un grand nombre de rameaux latéraux fournis par le nerf alors qu'il passe sur la face supérieure de la membrane branchiostège, tandis que le hyo-hyoïdien inférieur recoit la branche terminale du nerf, qui traverse entre le sixième et le septième rayon et pénètre donc immédiatement dans le muscle (v. page 87 et 88 ).

L'action du premier muscle est de rapprocher les rayons branchiostèges les uns des autres et de l'opercule et, par conséquent, de contribuer à l'ouverture de la fente branchiale; le 
second, comme l'indique VETTER, est toujours antagoniste, il écarte les rayons les uns des autres et tend à rapprocher l'arc hyoïdien de la ligne médiane, ce qui a pour résultat l'occlusion par en bas de la fente branchiale.

Comme on le voit, le muscle hyo-hyoïdien inférieur du Silure n'est pas un simple prolongement du muscle hyo-hyoïdien supérieur, "inclus dans la partie médiane de la membrane branchiostège » (VETTER, p. 516). Il a même une origine très étendue sur des parties osseuses, et ne reçoit du hyo-hyoïdien qu'un contingent de fibres insignifiant auprès de sa masse. En outre, une partie de son origine entoure celle du génio-hyoïdien, de sorte qu'il est difficile de dire où est la limite des deux muscles. Au premier abord, il semble que le génio-hyoïdien soit une portion superficielle d'un muscle dont le hyo-hyoïdien inférieur serait la portion profonde. Mais la connexion des origines ne signifie rien par elle-même, car elle peut être secondaire. En tout cas, elle n'a lieu que du côté médial du géniohyoïdien; en avant et latéralement, les deux origines ne peuvent pas être confondues. Enfin, le muscle profond n'entre aucunement en relation avec la mandibule; son insertion est strictement limitée à la pièce inférieure de l'arc hyoïdien, ce qui le caractérise donc bien comme muscle hyo-hyoïdien.

VETTER a montré que chez les Téléostéens examinés par lui, une forte portion du muscle génio-hyoïdien (la partie postérieure) serait innervée par le rameau terminal du nerf hyoïdien, ce qui n'a pas lieu chez le Silure (v. précédemment et p. 89). Il est donc probable que chez d'autres Téléostéens la connexion entre le hyo-hyoïdien inférieur et le génio-hyoïdien est plus étroite que chez le Silure, ou encore qu'une partie importante du hyo-hyoïdien inférieur s'est adjointe au génio-hyoïdien, et alors le hyo-hyoïdien inférieur de ces Téléostéens ne serait plus que le prolongement du supérieur. Dans ce cas, le Silure aurait conservé un état plus primitif.

Rev. Suisse de Zool., T. 6. 1898. 
Enfin, on peut encore insister sur les points suivants : le hyohyoïdien du Silure, par son origine sur le cérato-hyal, semble avoir conservé un état plus inférieur que celui des quatre Poissons osseux étudiés par VETTER. Par sa réunion médiane avec celui de l'autre côté, il se rapproche de l'état de Barbus, tandis que par la division du tendon médian, telle qu'elle a été décrite, il rappelle l'état de Esox, avec cette différence que chez ce dernier c'est toute la partie musculeuse qui se divise, tandis que chez Silurus, ce n'est qu'une faible partie tendineuse.

\section{B. Muscles des arcs branchiaux et de l'arc pharyngien.}

4. Pharyngo-arcuo-hyö̈dien (fig. 40, Pah).

Le muscle que je désigne ainsi ne correspond exactement à aucun de ceux décrits par les auteurs chez d'autres Téléostéens. Ce n'est pas non plus une formation nouvelle, mais bien un muscle qui a des liaisons avec les extrémités ventrales de tous les arcs, depuis le pharyngien inférieur jusqu'à l'arc hyö̈dien et qui réunit aussi chaque cérato-branchial (Cbr) à l'hypobranchial (Hbr) du même arc. Il cumule donc les propriétés des obliques ventraux, que VETTER a trouvés sous des états assez différents suivant les espèces, d'un pharyngo-hyoïdien, connu jusqu'à présent seulement chez la Perche, et d'un pharyngoarcual qui présente un aspect très différent chez le Brochet d'une part, et les Cyprins d'autre part, et manque complètement chez la Perche (voir 48, p. 517-521).

Ce muscle est très allongé; il atteint sa plus forte épaisseur dans sa moitié antérieure et il est constamment situé à quelque distance de la ligne médiane. C'est en arrière qu'il est le plus éloigné de cette dernière; il s'en rapproche peu à peu jusqu'au niveau du cérato-branchial II, à partir de là il lui est presque 
parallèle. Sa face inférieure est recouverte par la muqueuse des arcs, qui, dans cette région, porte encore des lamelles branchiales.

En arrière, il commence par un fort faisceau semi-tendineux, sur la partie horizontale de l'os pharyngien inférieur, latéralement par rapport à l'insertion du pharyngo-claviculaire externe. La partie latérale de ce faisceau va s'insérer sur la région voisine du cérato-branchial IV, à son bord postérieur; cette partie est assez nettement délimitée à la surface du muscle (fig. 10), mais on ne peut cependant la séparer que d'une manière incomplète. L'autre partie du même faisceau, la plus forte, passe au-dessous de l'origine du transverse pharyngien et se prolonge jusqu'à l'extrémité antérieure du muscle dont elle constitue le bord médial.

De l'extrémité antérieure du cérato-branchial IV, part un nouveau faisceau, moins considérable que celui qui a son origine sur le pharyngien inférieur. Il envoie une petite partie de ses fibres à la face postérieure de l'apophyse ventrale de l'hypobranchial III, tandis que le reste se prolonge jusqu'à l'extrémité antérieure du muscle. Entre la crête ventrale de l'hypobranchial III et celle de l'hypobranchial II, il y a un court faisceau, étendu dans le prolongement de la partie insérée sur la face postérieure de l'hypobranchial III.

La même disposition se répète pour les arcs situés en avant, avec cette différence que le faisceau naissant sur le cérato-branchial envoie une partie de ses éléments s'insérer sur la crête inférieure de l'hypobranchial correspondant. Les deux autres parties correspondent à celles du faisceau naissant sur le cératobranchial IV; la plus faible va à l'hypobranchial de l'arc précédent, la plus forte se prolonge jusqu'à l'extrémité antérieure du muscle. Enfin, dans le prolongement des deux parties insérées respectivement sur l'hypobranchial d'un arc et sur l'hypobranchial de l'arc précédent se trouvent aussi des fibres qui vont se fixer sur l'hypobranchial situé en avant. 
Du cérato-branchial I part aussi un faisceau. Celui-ci envoie toutes ses fibres au prolongement commun à tous les faisceaux d'origine, qui forme alors un fort muscle s'attachant en avant par un tendon cylindrique $\left(P a h_{t}\right)$ sur le bord supérieur et postérieur (interne) de l'hypohyal, près de la suture entre ce dernier et le cérato-hyal.

Si nous comparons les diverses parties de ce muscle à des muscles d'autres Téléostéens, nous voyons que les faisceaux naissant sur les cérato-branchiaux correspondent parfaitement aux obliques ventraux. La partie de ces faisceaux insérée sur l'hypobranchial du même arc correspond, chez le Brochet, à la partie que VetTen désigne par les lettres $\mathrm{Ov}$, (48, Pl. 13, fig. 9) et la partie insérée sur la pièce inférieure de l'arc précédent à $O_{v_{2}}$. Il n'y a que des différences peu importantes; ainsi chez le Silure, le faisceau du cérato-branchial IV n'envoie pas de fibres à l'hypobranchial IV (ce qui s'explique par le fait que le faisceau est séparé de ce dernier par le transverse pharyngien); toutes les fractions représentant les $\mathrm{Ov}_{2}$ sont charnues et non pas en partie tendineuses comme chez le Brochet. Le fort faisceau naissant sur l'os pharyngien inférieur correspond au pharyngo-arcual du Brochet et des Cyprins, qui se divise aussi en deux parties, l'une s'insérant sur le cérato-branchial IV, l'autre se réunissant à l'oblique ventral $d u$ quatrième arc pour se fixer à l'hypobranchial III. La seule différence est que, chez le Silure, cette dernière partie se prolonge plus en avant, jusqu'à l'insertion de l'arc hyoïdien et rappelle parfaitement le pharyngo-hyoïdien de la Perche (v. Cuvier, Pl. 5, nº 35).

La comparaison avec le Brochet peut être poussée plus loin encore en remarquant, avec VETTER, (p. 518), que chez ce Poisson, les parties $\mathrm{Ov}_{1}$ sont recouvertes immédiatement par la couche relativement bien développée des muscles interbranchiaux, et qu'en avant elles passent même directement dans 
ceux-ci. Chez le Silure, ces muscles interbranchiaux sont évidemment représentés par les faisceaux étendus entre les hypobranchiaux consécutifs, et que j'ai considérés comme des prolongements de ce qui correspond aux obliques ventraux. En outre, le Brochet possède une couche musculaire médiane étendue au-dessous de la deuxième et de la troisième copule (Vetter, fig. 9, x). Vetter en trouvait la signification énigmatique, mais il pensait que ce pouvait être une différenciation des obliques voisins. Chez le Silure, cette couche n'est pas médiane, elle est moins étalée, mais plus épaisse et unie au prolongement du faisceau naissant sur l'os pharyngien inférieur pour constituer la plus forte partie du muscle pharyngo-arcuohyoïdien, insérée sur l'hypohyal.

La comparaison avec trois catégories de muscles est donc bien justifiée. Les différences qui se présentent n'ont qu'une faible valeur; ce sont des variations secondaires dans le nombre et le volume, comme celles qui ont été indiquées plus haut, ou dans l'insertion: par exemple le pharyngo-hyoïdien très volumineux de la Perche est fixé sur l'uro-hyal, tandis que le faisceau correspondant du Silure a son insertion avec les autres faisceaux sur l'hypohyal. Les différentes parties du muscle, si elles peuvent être ainsi comparées à des muscles distincts chez d'autres Poissons, n'en sont pas moins en étroite connexion les unes avec les autres, et jamais la séparation complète ne peut s'effectuer naturellement. Même les faisceaux d'origine sont très vite fusionnés d'une manière complète avec la masse commune.

L'innervation se fait par les prolongements des rameaux branchiaux du glosso-pharyngien et du vague (fig. 10, rbg, rbv). L'introduction de filets de la branche pharyngienne inférieure dans le faisceau pharyngo-arcual naissant sur l'os pharyngien inférieur n'a pu être constatée d'une manière absolument certaine, à cause de la proximité des deux pharyngo-claviculaires et du transverse pharyngien. Elle est cependant très pro- 
bable; plusieurs filets traversant ces derniers ou passant à leur surface ont pu être suivis jusque sur le muscle.

Ce muscle tire l'appareil branchial en avant, en comprimant les uns contre les autres tous les arcs, y compris l'arc pharyngien incomplet.

\section{Transverse ventral (fig. 10, Tv).}

Muscle court, épais, naissant sur l'extrémité ventrale du cérato-branchial IV, sur sa face inférieure et médiale, mais recevant aussi un faisceau postérieur de la partie du pharyngoarcuo-hyoïdien qui naît sur l'os pharyngien inférieur. Les fibres sont parfaitement transversales et se fixent sur le cérato-branchial IV de l'autre côté. Seul, le faisceau venant de l'os pharyngien va d'abord d'arrière en avant contre la face latérale du pharyngo-claviculaire externe, puis s'infléchit médialement devant le bord antérieur de celui-ci; à partir de là, il est tout à fait transversal. Ce faisceau ne représente qu'une très faible partie du transverse ventral dont on peut, du reste, le séparer facilement. Je dois ajouter qu'il a été observé sur un gros exemplaire, et qu'il n'a pas été trouvé sur un individu plus petit (fig. 10).

L'innervation a lieu par un filet terminal de la branche postérieure du troisième tronc branchial ou nerf antérieur du quatrième arc.

Ce muscle rapproche de la médiane les deux cérato-branchiaux IV. Il dilate les interstices entre les arcs; c'est donc un antagoniste du muscle précédent.

6. Pharyngien transverse (fig. 10,Pht).

Il nait sur la face inférieure de l'arc pharyngien incomplet, à peu près sur son tiers antérieur. Ses fibres sont dirigées 
obliquement en avant et médialement, et se réunissent avec celles de l'autre côté sur la ligne médiane en formant, au moins en avant, une inscription tendineuse. C'est donc une couche musculaire étendue. Elle est plus épaisse dans la partie antérieure que dans la partie postérieure; sa face inférieure forme le plafond de la cavité où est logé le cœur et son bord latéral est longé ventralement par le pharyngo-claviculaire interne. En arrière, le pharyngien transverse touche au constricteur du pharynx $(C p h)$, qui ne s'en distingue que par la direction moins oblique de ses fibres.

Le pharyngien transverse est innervé par un rameau de la branche pharyngienne inférieure du vague; ce rameau envoie probablement aussi quelques filets dans la partie postérieure (pharyngo-arcuale) du pharyngo-arcuo-hyoïdien.

L'action de ce muscle, renforcée par celle du transverse ventral et des muscles dorsaux dont il a été question plus haut, consiste à écarter les arcs branchiaux les uns des autres en tirant médialement l'os pharyngien inférieur.

\section{Pharyngo-claviculaires (tig. 10, Pce et Pci).}

Le pharyngo-claviculaire externe prerd naissance sur la face supérieure de la clavicule, du côté latéral, à peu près au tiers antérieur de la partie ventrale de cet os. Son origine $\left(P c e_{o}\right)$ est enchâssée entre les fibres du sterno-hyoïdien, qui naissent aussi sur la face supérieure de la clavicule. Il se dirige en haut, un peu médialement et en avant; sa face médiale forme une partie de la paroi latérale de la cavité logeant le cour. L'insertion $\left(P c e_{i}\right)$, par un tendon court, mais épais, a lieu sur la face inférieure de l'os pharyngien inférieur, vers le bord latéral, entre l'origine du transverse pharyngien et celle du pharyngo-arcuo-hyoïdien.

Ce muscle tire l'arc pharyngien en bas et légèrement en arrière. 
Le pharyngo-claviculaire interne est aussi un muscle puissant ; il est moins épais et plus long que l'externe. Son origine est aussi sur la face supérieure de l'os claviculaire, mais plutôt du côté du bord médial, plus en arrière et plus haut que celle du muscle précédent, soit environ au tiers postérieur de la partie ventrale de l'os. Il commence par un mince ruban tendineux occupant à peu près le quart de la longueur totale du muscle; il va en avant et un peu médialement, en s'élevant faiblement. Sa face supérieure recouvre ventralement le bord latéral, c'està-dire l'insertion du transverse pharyngien; sa face inférieure forme, en partie, le plafond de la cavité logeant le cœur. En avant, il croise médialement l'extrémité supérieure du pharyngoclaviculaire interne et passe alors au-dessous du bord antérieur du transverse pharyngien et au-dessus du bord postérieur du transverse ventral et, se prolongeant un peu sur la face dorsale de ce dernier, il s'insère sur l'extrémité antérieure de l'os pharyngien inférieur. L'insertion semi-tendineuse $\left(P c i_{i}\right)$ se trouve devant celle du muscle externe et plus médialement; elle est très rapprochée de celle du congénère symétrique, mais n'entre pas en contact avec elle.

Ce muscle tire l'arc pharyngien, et par conséquent les arcs branchiaux, en arrière et un peu en bas, aidé dans cette action par des muscles dorsaux qui tirent l'appareil en arrière en l'élevant un peu. Il fonctionne donc comme antagoniste principal du pharyngo-arcuo-hyoïdien.

Les pharyngo-claviculaires du Silure ne sont sûrement pas innervés par la branche antérieure du nerf occipital comme M. Furbringer (99) et Mac Murrich (72) l'indiquent pour d'autres Poissons osseux, mais bien comme Vetter l'a trouvé pour Esox et Perca, par la branche pharyngienne inférieure (fig. 3, 4,10, phi). Dans l'exemplaire que j'ai sous les yeux, le rameau principal de la branche, après émission des rameaux pour la muqueuse du pharynx, pour le transverse pharyngien et pro- 
bablement aussi pour la partie postérieure du pharyngo-arcuohyoïdien, pénètre dans le muscle interne, vers le milieu de sa longueur, à son bord médial (supérieur). Il s'avance horizontalement dans le muscle en lui abandonnant des filets, en sort plus loin, à son bord latéral (inférieur), un peu en avant du point où ce dernier croise le bord postérieur du pharyngo-claviculaire externe, pénètre dans ce muscle par sa face médiale et s'y ramifie. Le rameau décrit ici ne semble pas avoir de relations assez étroites et nombreuses avec des rameaux dépendant du nerf occipital ou des premiers nerfs spinaux, pour que l'on puisse douter de son origine.

Il reste inexpliqué que ces deux muscles, qui semblent présenter une grande constance parmi les Téléostéens, soient innervés tantôt par la branche antérieure du dernier nerf cranien, tantôt par la branche pharyngienne inférieure du nerf vague; mais l'existence de ces deux modes d'innervation semble d'autant plus probable que, si VETTER a trouvé le premier chez Esox et Perca, il a trouvé le second chez Cyprinus, au moins pour une partie du muscle interne (48, p. 524).

\section{Musculature longitudinale ventrale}

1. Sterno-hyoüdien (Pl. 3, fig. 10, Sth).

C'est, chez les Téléostéens, le seul représentant de la musculature longitudinale ventrale. Chez le Silure, il est très épais en arrière et naît presque exclusivement sur la face supérieure de la partie ventrale de la clavicule. L'origine $\left(S t h_{0}\right)$ commence un peu plus en avant que celle du pharyngo-claviculaire interne, mais vers le bord latéral, et occupe, jusqu'à l'extrémité antérieure, la surface de l'os tournée latéralement. Le muscle ventro-latéral du tronc se fixe sur la face inférieure de la partie ventrale de l'os claviculaire, et il n'y a qu'une mince couche 
superficielle de ses fibres qui passe sous le bord inférieur de l'os pour s'ajouter au sterno-hyoïdien.

Le muscle a la forme d'une pyramide à trois faces, une des faces est couverte par la muqueuse de la cavité branchiale, une autre par la peau de la face ventrale, et la troisième est appliquée contre le muscle congénère symétrique dont elle n'est séparée que par son fascia. En avant, la face médiale forme une lame tendineuse qui s'insère sur la crête sagittale de la face supérieure de l'uro-hyal (os hyö̈de); en outre, le muscle, sans former de tendon distinct, s'attache par sa pointe sur l'uro-hyal (Uhy) dans l'angle formé par la face supérieure et la crête verticale de cet $o$ s. En avant, l'os lui-même est solidement réuni à l'hypohyal par deux forts ligaments cylindriques (LUhy) qui se touchent médialement.

Le sterno-hyoïdien est pourvu par le rameau hypoglosse (rhpg) ou branche antérieure d'un nerf formé par le nerf occipital et une partie du premier nerf spinal et qui se bifurque vers le bord latéral inférieur du pharynx. Il tire l'os hyoïde et la mandibule en arrière et fonctionne donc comme antagoniste de l'adducteur mandibulaire.

\section{Musculature céphalo-scapulaire}

Il n'y a pas de muscle spécial indépendant, reliant le crâne à la ceinture scapulaire ou à l'épine dorsale.

JAQUET mentionne et représente, dans sa monographie de la Perche (90, p. 507, fig. 208, oc) un occipito-claviculaire « entre l'occiput, le pharyngien supérieur et la ceinture scapulaire ». Ce muscle doit correspondre à ce que $\operatorname{CuviER}(7$, p. 288, $\mathrm{Pl}$. V , y) a indiqué comme « lambeau » du muscle latéral du tronc, partant de la première côte et allant jusqu'à l'os mastoïdien.

Chez le Silure, nous trouvons un muscle indépendant dans la même situation, semble-t-il, que ce muscle occipito- 
claviculaire, mais sans relation avec le pharyngien supérieur, ni avec la région occipitale proprement dite. Son origine s'étend sur la face inférieure du supra-claviculaire (fig. 1, 2, 5, Sc), os qui s'appuie, en avant et en haut, sur l'angle postéro-latéral du cràne, soit sur l'exoccipital(Exo) et s'articule en arrière avec l'extrémité supérieure de la clavicule $(\mathrm{Cl})$; quelques fibres sont parfois repoussées jusque sur la partie voisine de l'exoccipital, mais cette extension n'est probablement que secondaire.

L'insertion se trouve sur la partie supérieure de la clavicule, à sa face antérieure, et va environ jusqu'à la moitié de la distance entre l'extrémité dorsale de cet os et son articulation avec la pectorale.

Ce muscle doit élever l'os claviculaire en le tirant en avant et médialement. Il n'a pas son origine sur le crâne et ne peut être compté comme partie de la musculature céphalique; il appartient exclusivement à la musculature scapulaire et mériterait mieux le nom d'élévateur claviculaire. Cependant, il nous à semblé utile de le mentionner ici, car il est innervé par un rameau sortant du tronc pharyngo-intestinal du vague. Il est partiellement visible dans la figure 5 (Pl. $\left.3, E_{c}\right)$.

\section{Prolongement du muscle dorso-latéral (fig. 5, Mdl).}

La seule partie musculaire reliant le tronc au crâne est le prolongement antérieur du muscle latéral. Ce prolongement est constitué, en majeure partie, par la portion dorsale, mais il s'y ajoute un faisceau venant de la partie ventrale. Des explications précises seront données plus tard sur cette musculature, à propos du trajet des nerfs.

Il suffira de remarquer, pour le moment, que cette masse musculaire est très considérable, qu'elle est paire jusqu'à sa partie antérieure, c'est-à-dire que les deux parties symétriques se touchent par leur fascia, sans se fusionner. Elle recouvre 
toute la moitié postérieure du couvercle cranien, où elle s'avance sur le commencement du frontal principal jusqu'à une ligne en relief indiquée dans la figure 2 comme ligne nucale (Ln).

Ce muscle tire la tête à droite ou à gauche et un peu en haut, mais ce dernier mouvement ne peut être qu'excessivement faible, vu la connexion entre les premières vertèbres et entre la crête occipitale et l'arc supérieur des deuxième et troisième vertèbres réunies.

L'innervation a lieu par les premiers nerfs spinaux.

\section{NERFS CÉREBBRAUX}

La bibliographie concernant les nerfs cérébraux des Poissons est très vaste; nous n'en donnerons pas ici un aperçu général, c e serait un long exposé dont on ne pourrait retirer qu'un faible profit. Nous traiterons les nerfs craniens du Silure dans l'ordre suivant et nous donnerons les indications de la littérature au fur et à mesure, dans tous les cas où elles pourront être utiles.

I. - Nerf olfactif.

II. - Nerf optique.

III. - Nerfs oculo-moteurs.

IV. - Nerfs trijumeau et facial.

V. - Nerf acoustique.

VI. - Nerfs glosso-pharyngien et vague.

VII. - Nerf occipital.

\section{Nerf OLFACTIF}

(Pl. 2, fig. 4, nol.)

On sait depuis longtemps que le nerf olfactif des Poissons se présente sous deux formes. Tantôt il sort d'un lobe ou bulbe olfactif situé en avant du prosencéphale et traverse la cavité orbitaire, tantôt il sort d'un bulbe olfactif placé à l'entrée de la fosse nasale et relié au cerveau par un pédoncule plus ou 
moins long, que Gegenbaur (35) et SAgemenl (63) ont appelé tractus olfactif. Ce dernier auteur a montré que c'est le second état que l'on doit considérer comme primitif.

Le nerf olfactif de Silurus glanis appartient à la deuxième forme. Le tractus olfactif ( $\mathrm{t} 0$ ) sort du prosencéphale par un faisceau cylindrique peu épais. D'abord rapproché de la ligne médiane du cerveau, il s'en éloigne bientôt et vient côtoyer la paroi de cette sorte d'étui osseux qui prolonge la cavité cranienne jusqu'à la région rostrale. Il parvient dans la partie antérieure de cet étui, laquelle est divisée en deux par une cloison cartilagineuse médiane dépendant de l'ethmoïde (vue en section horizontale dans la fig. 6, Ceth), puis traverse la paroi pour pénétrer dans la fosse nasale (fig. 3, 4, 6, N). A cet endroit, il forme un renflement dont le diamètre est trois à quatre fois plus fort que le sien et qui correspond au bulbe olfactif (Stannius, 16, p. 3 et 4, Baudelot, 38, p. 83). De ce renflement part le nerf olfactif proprement dit, lequel est excessivement court et se partage bientôt en deux ramifications placées l'une à côté de l'autre, dans le prolongement du tractus et dans l'axe longitudinal de la fosse nasale. De chacune des partiés sortent de nombreux et courts filets destinés aux lamelles olfactives.

Le long prolongement de la cavité cranienne qui loge le tractus olfactif est limité en bas par le parasphénoïde (fig. 1, Ps), en haut par le frontal principal (Fr) et l'ethmoïde (Eth), latéralement par l'alisphénoïde (Al) et le préfrontal (Prf). Le tractus n'est qu'une partie de l'organe central.

\section{NERF OPtique}

(Pl. 2, fig. 3, 4, op.)

De même que le tractus olfactif, le tractus optique est très long. Sa faible épaisseur est en rapport, d'une part avec les dimen- 
sions de l'œil, placé en avant et au bord latéral de la face frontale, d'autre part avec la grosseur dés lobes optiques, qui sont presque entièrement recouverts par le cervelet.

Les deux nerfs sortent de la face inférieure des lobes optiques, à peu près à la limite entre ceux-ci et les lobes inférieurs. Ils apparaissent de chaque côté du trigonum fissum sous la forme de deux petites saillies qui deviennent bientôt indépendantes de la base du cerveau. Les deux nerfs viennent converger en avant du court pédoncule de l'hypophyse et se croisent en ce point, de telle manière que le nerf issu du lobe optique gauche et se rendant à l'œil droit passe sous le nerf issu du lobe optique droit. En arrière de ce croisement dans lequel il n'y a aucun échange de fibres, se trouve une commissure entre les deux nerfs, la commissure de HALLER.

Après le croisement, chaque nerf est d'abord légèrement plus rapproché de la ligne médiane que le tractus olfactif, mais croise bientôt celui-ci pour venir longer la paroi de la cavité cranienne. Il sort du crâne par un trou de la partie antérieure de l'alisphénoïde ( $\mathrm{Pl}$. 1, fig. 1, op). Son trajet, à partir de ce point jusqu'à l'œil, représente encore plus de la moitié du trajet total; il est alors entouré d'un névrilemme résistant, puis, comme d'une seconde gaîne, par les quatre muscles oculaires droits prenant naissance en arrière autour de l'orifice de sortie du nerf.

Le Silure n'a pas de cavité orbitaire proprement dite. Depuis sa sortie du crâne, l'optique est englobé dans une masse de graisse qui enveloppe aussi de nombreux nerfs plus faibles, destinés soit aux muscles oculaires, soit au globe de l'œil, soit à la peau, et se trouve placé au-dessus du nerf maxillaire inférieur, entre le bord médial de l'adducteur mandibulaire profond et la face interne de l'élévateur palatin (fig. 5, 6). Il pénètre dans le globe de l'œil, un peu au-dessus de l'axe de celui-ci et un peu de côté, dans la direction du muscle droit interne. 


\section{Nerfs OCULO-MOTEURS}

(Pl. 2, fig. 4, en rouge.)

Les nerfs de la $\mathrm{III}^{\mathrm{e}}, \mathrm{IV}^{\mathrm{e}}$ et $\mathrm{VI}^{\mathrm{e}}$ paires sont représentés chez. le Silure par des filets excessivement ténus.

Ils sortent séparément du cerveau. Le premier, l'Oculomoteur commun, émerge de la moelle allongée par un seul faisceau, immédiatement derrière le lobe inférieur, s'infléchit latéralement pour contourner ce dernier, puis se dirigeant en avant, vient se placer contre le bord interne et inférieur du tronc du trijumeau-facial. Je suivant, le trochléaire, est le plus grêle des oculo-moteurs. Il sort, comme chez les autres Poissons osseux, de la partie postérieure du lobe optique, c'est-à-dire du sillon qui sépare ce dernier du cervelet. Il se dirige obliquement en avant et descend contre la face interne du complexe trijumeaufacial et là, il m'a paru s'appliquer contre le nerf de la III ${ }^{\mathrm{e}}$ paire. Il est très fin et s'est brisé au moment où je voulais m'assurer de ce fait sur une préparation où les deux autres nerfs et leurs ramifications avaient été obtenus en entier. Enfin l'abducteur ou nerf de la VI ${ }^{\mathrm{e}}$ paire sort, également par un faisceau unique, de la face ventrale de la moelle allongée, non loin de la ligne médiane, donc près de son congénère de l'autre côté. Il se dirige en avant et se place aussi contre le tronc du trijumeau-facial, au-dessous de l'oculo-moteur commun.

Donc, à la face interne de la partie intra-cranienne du trijumeau, on peut voir deux filets nerveux superposés et appliqués contre ce tronc nerveux, jusqu'à une petite distance de sa sortie du crâne. Ils s'en séparent alors, continuent à aller en avant en s'éloignant un peu l'un de l'autre, le plus faible étant placé légèrement plus bas et plus médialement. Ces deux filets quittent la boite cranienne par deux orifices distincts, situés dans l'alisphénoïde ( $\mathrm{Al}$ ) entre ceux du trijumeau et de l'optique, plus bas 
que ceux-ci. Le trou antérieur (fig. $1, o m+\operatorname{trl}$ ) donne passage au filet supérieur formé très probablement par l'oculo-moteur commun et le trochléaire réunis, le postérieur au filet inférieur. Les deux filets sont accompagnés à leur sortie d'une gaîne résistante qui les rend difficiles à préparer.

Le filet inférieur, le plus mince, se rapproche beaucoup du nerf optique et vient cheminer contre le bord latéral de celui-ci. Il se divise en deux parties d'inégale grosseur, qui pénètrent toutes deux dans la région moyenne du muscle droit externe, soit dans celle qui fait suite à la portion effilée et tendineuse fixée sur le crâne. D'après sa distribution, ce nerf est donc bien l'abducteur ou nerf de la $I V^{\mathrm{e}}$ paire (fig. 4, abd).

Le filet supérieur, quoique faible, est plus fort que le filet inférieur. A environ $1 \mathrm{~cm}$. de sa sortie du crâne, il se bifurque en un rameau supérieur et en un rameau inférieur, qui vont se diviser à leur tour. Le premier donne deux filets excessivement fins, dont l'un se rend au muscle oblique supérieur, près de son insertion sur le globe oculaire et représente le nerf trochléaire (fig. 4, trl) l'autre entre dans la partie postérieure du muscle droit supérieur et n'est qu'une branche de l'oculo-moteur commun (omds). Le second rameau constitue la plus grande partie du nerf oculo-moteur commun (fig. 4, om). Il est d'abord placé au-dessus du nerf abducteur, puis il descend entre le nerf optique et le muscle abducteur (c'est jusqu'à cet endroit qu'il est représenté dans la fig. 4) et parvient ainsi au-dessus du nerf optique. Il se divise alors en deux parties : l'une se termine par deux filets allant dans le muscle oblique inférieur et le muscle droit interne, l'autre pénètre dans la région postérieure du muscle droit inférieur.

Stannius, dans son ouvrage fondamental sur le système nerveux périphérique des Poissons (16), dit qu'il y a généralement un nerf oculo-moteur pour les muscles droit supérieur, droit inférieur, droit interne et oblique inférieur, un trochléaire pour 
l'oblique supérieur et un abducteur pour le muscle droit externe. Puis il ajoute quelques remarques au sujet de différences particulières à certaines espèces, et spécialement au Silure. Chez ce Poisson, il voit le nerf oculo-moteur quitter la cavité cranienne par une ouverture qui lui est propre " tandis que les fines racines des nerfs trochléaire et abducteur s'appliquent immédiatement contre le nerf trijumeau, de sorte que si l'on n'a pas recherché leurs racines et les points d'origine de celles-ci, on doit regarder les nerfs se rendant aux muscles oblique supérieur et droit externe comme des rameaux du $R$. primus N. trigemini $»$ (branche ophtalmique).

Le résultat de nos observations diffère notablement de ces indications, mais tout porte à croire que les rameaux décrits par Stannius comme nerfs oculo-moteurs ayant passé dans la voie de l'ophtalmique $(16$, p. 35$)$ ne sont autre chose que des rameaux de l'ophtalmique, qui se détachent déjà lors de son passage à travers la paroi du crâne et qui seront étudiés plus loin comme filets du rameau oculo-nasal. Plusieurs de ces filets sont en effet très fins, mais cependant plus forts que les moteurs oculaires; ils passent à la surface des muscles de l'œil et semblent même parfois pénétrer dans leur épaisseur. En réalité, ils ne font qu'adhérer très fortement à la périphérie de ces muscles et, arrivés à leur partie antérieure, ils se replient en arrière ou se prolongent en avant pour se distribuer dans le tégument situé autour de l'œil ou dans l'enveloppe de l'œil. La probabilité de cette erreur est rendue encore plus grande lorsqu'on remarque que STANNIUs n'indique pas précisément s'il a pu suivre les filets qu'il appelle trochléaire et abducteur de leur naissance jusqu'à leur sortie du crâne. En outre, il ne parle pas des filets dépendant de l'ophtalmique, qui s'appliquent contre les muscles oculaires et se perdent dans le tégument qui environne l'œil.

La recherche des nerfs oculo-moteurs vers leur sortie du Rev. SUISSE DE ZooL., T. 6. 1898. 
crâne est, en effet, rendue très difficile par leur extrême finesse et leur proximité du trijumeau. Une préparation heureuse m'a cependant donné la certitude qu'il existe un nerf abducteur très grêle, complètement indépendant, placé au-dessous d'un nerf qui se ramifie et envoie des filets à tous les muscles de l'œil sauf au muscle droit externe. Je n'ai pu découvrir de nerf complètement indépendant pour le muscle oblique supérieur; ce muscle est actionné par un rameau qui semble sortir de l'oculo-moteur commun et qui n'est sans doute que le nerf trochléaire uni à l'oculo-moteur dans la cavité cranienne. Enfin, l'oculo-moteur commun fournit un filet qui pénètre dans le globe oculaire audessous du nerf optique. Je n'ai pu trouver aucune communication entre ce filet et le rameau de l'ophtalmique qui entre aussi dans l'œil, pas plus qu'un ganglion ciliaire. Des recherches spéciales comme celles de Schwalbe (54) et ScHNEIDER (60) pourraient seules élucider complètement ce point.

\section{Nerfs triJumead ET FaCIAL}

Sortie du cerveau et ganglion de Gasser. Sous le rapport de leurs origines, ces deux nerfs doivent être traités ensemble. Ceci se trouve déjà indiqué par le fait que d'anciens auteurs comme Desmoulins (1825), Cuvier et Valenciennes (1828), BüchNer (1835) ont regardé comme une simple branche du trijumeau, appelée par eux branche operculaire, le tronc nerveux qui, d'après les recherches récentes, doit contenir la majorité des éléments du facial. Du reste, en 1845, CuviER admettait que la branche operculaire est constituée par le facial.

STANniUs (1849), s'appuyant sur des recherches microscopiques, établit que les deux nerfs sont formés de cinq racines (moins souvent de quatre, rarement de trois par juxtaposition ou réduction) très rapprochées les unes des autres. De ces cinq racines, les unes envoient tous leurs éléments à l'un des deux 
nerfs, les autres les envoient aux.deux nerfs. Enfin, les ganglions qu'elles forment sont souvent reliés entre eux et peuvent même se fusionner.

BAUDELOT (1883, œuvre posthume), voit le trijumeau naître de la moelle allongée par une racine antérieure formée de deux faisceaux et une racine postérieure formée de trois faisceaux. Parmi ces trois derniers, le faible faisceau postérieur est un filet moteur « et nous devons le considérer ou comme le représentant du facial, ou comme l'équivalent de la racine motrice du trijumeau chez les Maminifères » (p.53). Les éléments de ce faisceau vont dans une branche que l'auteur n'appelle pas branche operculaire, ni même facial, parce qu'une portion de cette branche seulement se rend à l'opercule et qu'elle tire presque en totalité son origine du trijumeau (p. 125); il la désigne comme branche descendante postérieure du trijumeau.

Le «groupe du trijumeau », d'après GegEnbaur $(31 ; 1874)$ comprend des nerfs distincts, les nerfs moteurs oculaires, et une masse volumineuse formée de quatre (rarement trois ou cinq) racines réunies en un plexus ganglionnaire. La partie antérieure de cette masse constitue le trijumeau proprement dit; la partie postérieure appartient au facial, lequel représente la racine motrice d'un nerf ayant le type des nerfs spinaux et dont la racine sensible serait le nerf acoustique. Une partie du facial forme, avec une branche venue du trijumeau, le rameau operculaire, tandis que le reste du facial se continue comme rameau hyo-mandibulaire.

Friant (1879) comme BAUdelot, voit le trijumeau constitué par deux racines, mais il attribue à celles-ci une valeur morphologique différente. La racine antérieure est faible et formée d'un faisceau unique; la racine postérieure se décompose généralement en deux faisceaux (ce nombre augmente ou diminue par disjonction d'un des faisceaux ou par fusion des deux), lesquels correspondent aux deux faisceaux postérieurs de BAU- 
DELOT, tandis que, du faisceau antérieur du même auteur, FRIANT fait naître le nerf facial. Pour lui, la branche descendante postérieure du trijumeau (BAUDELOT) est complètement indépendante du facial; elle ne dépend absolument que du trijumeau dont elle est la branche mandibulo-hyoüdienne.

Wiedersheim (1893, p. 286), indique que le facial a primitivement une origine commune avec l'acoustique, mais qu'il s'en sépare secondainement et que, chez les Téléostéens en particulier, ses racines forment, avec celles du trijumeau, une masse commune nou séparable dans les préparations.

Goronowitsch $(1888,1897)$ publie deux travaux qui étendent aux Ganoïdes cartilagineux et aux Poissons osseux la théorie développée par GEGENBauR sur la métamérie des nerfs craniens des Sélaciens. Ses recherches portent principalement sur les centres d'origine et l'histologie des racines; le trajet périphérique est traité d'une manière plus succincte. D'après ces recherches, soit chez Acipenser ruthenus (81) soit chez Lota vulgaris (100), le trijumeau est formé de deux nerfs homodynames de nerfs spinaux, naissant chacun par une racine dorsale à fibres fines, portant un ganglion, et par une racine ventrale à fibres épaisses. Le facial est homodyname d'un seul nerf spinal et possède donc une racine sensible et une racine motrice.

Chez Acipenser, chacun des trois nerfs (trijumeau I, trijumeau II et facial) sort de la moelle allongée par deux racines distinctes qui se rendent dans une masse ganglionnaire où chaque nerf conserve cependant son individualité; il n'y a, entre ces nerfs, que quelques communications peu importantes. Chez les Poissons osseux (Lota et Esox), il se produit des réunions de racines à l'intérieur même du cerveau, non loin des centres d'origine; de plus, il arrive, entre autres particularités, que la racine motrice d'un nerf peut arriver dans le tronc du nerf suivant. Il en résulte que ces nerfs sortent de la moelle allongée, non plus sous la forme de « racines", dans le sens 
strict du mot, mais sous la forme de trones senso-moteurs. Des trois nerfs, le facial seul montre encore deux racines distinctes à sa sortie du cerveau. Enfin, dans le renflement ganglionnaire, ou immédiatement après, les troncs se divisent et se répartissent de telle manière dans les voies périphériques, que chacune d'elles renferme des fibres de deux ou mème de trois nerfs segmentaux.

Dans le tableau suivant, j'essayerai de résumer, d'après les résultats de GoRONOWITSCH, la répartition des trois nerfs primitifs (trijumeau I, trijumeau II et facial) dans les branches du complexe de Lota vulgaris :

\section{Trijumeau antérieur}

Racine dorsale.

Racine ventrale.

1. Ophtalmique profond. Ophtalmique profond.

2. Maxillaire supér ${ }^{\mathbf{r}}$ proprement dit.

Maxillaire supérieur proprement dit. 3 .

4. Maxillaire inférieur. Maxillaire inférieur.

5 .

6. Tronc hyoïdeo-mandibulaire.

7. Nerf de W WBer.

Nerf de Weber.

\section{Trijumeau postérieur}

Racine dorsale.

1. Ophtalmique superficiel.

2.

3. Nerf rostral ${ }^{1}$.

4.

5 .

6. Tronc hyoïdeo-mandibulaire.

7.
Racine ventrale.

Ophtalmique superficiel.

Tronc hyoïdeo-mandibulaire.

Nerf de Weber.

FACIAL

Racine dorsale.

Racine ventrale.

1. Ophtalmique profond.

2.

3 .

4. Maxillaire inférieur.

5. Palatin.

6. Tronc hyoïdeo-mandibulaire.

7. Nerf de Weber.

Trone hyoïdeo-mandibulaire.

Nerf de Weber.

${ }^{1}$ Ce nerf rostral forme, avec le nerf maxillaire supérieur proprement dit, le nerf maxillaire supérieur de Stannius. 
Si j'ai donné un compte rendu un peu étendu des recherches importantes de GoRoNowiTsch, c'est qu'elles confirment l'idée que chez les Poissons osseux, les branches nerveuses périphériques portant le même nom que chez des Poissons inférieurs ou les Vertébrés supérieurs ne leur sont pas parfaitement homologues. Par leur distribution périphérique, ces branches le font souvent prévoir, car, au lieu d'être purement sensorielles ou motrices comme leurs homonymes, elles se montrent mixtes à des degrés divers. Maintenant, nous savons exactement que l'échange des fibres se fait non seulement entre la racine dorsale et la racine ventrale de chacun des nerfs, mais d'une manière compliquée entre les troncs des trois nerfs segmentaux formant le complexe.

Le trijumeau-facial du Silure sort de la face latérale de la moelle allongée apparemment par un tronc unique. Ce tronc est d'abord relativement mince; sur un grand exemplaire, il mesurait environ 3 millimètres dans le sens dorso-ventral, ce qui représente son plus grand diamètre. Cette partie mince est courte. Bientôt le tronc s'épaissit beaucoup et son diamètre dépasse le double de ce qu'il était auparavant; il va, du reste, en augmentant légèrement durant tout le trajet intra-cranien. Une section faite dans cette région, qui est la masse ganglionnaire du complexe, serait de forme à peu près triangulaire, la base du triangle étant appuyée sur le plancher du crâne.

Malgré son apparence compacte, le tronc du trijumeau-facial peut ètre assez facilement décomposé en trois portions, surtout si on l'a laissé séjourner suffisamment dans une solution à $10 \%$ d'acide nitrique. Jamais l'une quelconque de ces parties ne peut être complètement isolée des autres depuis la sortie du cerveau jusqu'à l'extrémité distale du renflement ganglionnaire. C'est dans l'intérieur de ce dernier que les parties, d'ailleurs très étroitement unies par du tissu conjonctif, font de nombreux échanges de fibres, d'où l'impossibilité de séparer exactement les divers composants. 
On peut constater que la partie inférieure et externe du tronc, celle située contre l'angle formé par le plancher et la paroi de la cavité cranienne, est occupée par une très forte portion dont la délimitation est quelquefois rendue plus ou moins visible par une strie sur la face latérale du tronc. Ce groupe d'éléments entre presque entièrement dans le tronc hyoïdeo-mandibulaire, mais il ne le constitue pas exclusivement; il forme aussi, en grande partie, le nerf dorsal ou nerf de WEBER. A sa face inférieure, cette portion comprend un faible faisceau que l'on peut voir émerger de la moelle allongée immédiatement en avant de la portion antérieure de l'acoustique. C'est certainement la racine motrice du facial, laquelle conserve, comme chez la Lotte (100), une certaine indépendance.

La région inférieure et interne du plexus est occupée par une portion volumineuse, qui forme principalement la partie inférieure des deux nerfs maxillaires et ne peut être isolée que sur un court trajet, dans la partie proximale du ganglion commun.

La troisième portion, la plus considérable, est située au-dessus de la ligne de contact des deux autres portions. Elle renferme des éléments pour la partie supérieure des deux nerfs maxillaires, ainsi qu'une quantité plus faible pour le tronc hyoïdeomandibulaire et pour le nerf dorsal.

Enfin, il y a apparemment une quatrième portion, moins forte, placée dorsalement par rapport à la précédente et formant l'arête supérieure du tronc. Elle contient les éléments allant dans les branches ophtalmiques. Ses rapports avec les autres portions montrent que ce n'est pas une véritable portion du complexe, mais le produit d'union de faisceaux des deux portions précédentes, déjà distinct du reste des éléments avant la sortie du crâne.

Chacune des parties qui viennent d'être décrites participe plus ou moins au renflement ganglionnaire. La première, celle qui fournit au tronc hyoïdeo-mandibulaire la majorité de ses fibres, 
ne présente qu'un faible renflement; son épaisseur, assez considérable, reste à peu près la même dans toute sa longueur. La racine ventrale du facial, qui lui est intimément liée, n'y prend en effet aucune part (v. Stannius, p. 33). Les autres parties montrent un épaississement assez considérable, mais elles n'atteignent pas leur dimension maximum à la même distance du cerveau; c'est pour cela que le ganglion de GAssER s'étend depuis le voisinage de la moelle allongée jusqu'à la sortie du crâne et qu'il n'augmente qu'insensiblement d'épaisseur d'arrière en avant.

Division en branches et sortie du crâne. - Immédiatement après la formation du ganglion de GASSER, le tronc du trijumeau-facial se résout en plusieurs branches. L'une d'elles, cependant, quitte le plexus ganglionnaire à sa partie postérieure, non loin du cerveau. On la voit s'élever verticalement contre la face médiale (interne) du tronc, puis se diriger en arrière sous le plafond du crâne qu'elle traverse dans la région occipitale. Elle sera désignée plus loin comme nerf dorsal ou nerf de Weber (Pl. 2, fig. 3, 4, nWb). En avant de ce dernier partent plusieurs petits nerfs qui seront décrits comme rameaux ascendants antérieurs (rasc).

Plus distalement, se détache de la face latérale du plexus le tronc volumineux, nommé tronc hyoüden-mandibulaire ou facial (Stannius) (hmd). Il quitte la cavité cranienne par un canal de l'os pétreux $(\mathrm{Pe})$, dirigé en avant et un peu latéralement.

Les autres branches du complexe restent plus ou moins dans la même direction que le tronc du trijumeau-facial. La plus importante sort de la partie inférieure et antérieure de la masse ganglionnaire; elle constitue le tronc maxillaire commun, et traverse la paroi du crâne par un orifice situé dans l'os pétreux, près de l'alisphénoïde. La partie supérieure du ganglion donne naissance à deux branches, dont l'une, la branche ophtalmique profonde (opr), descend un peu et s'applique sur le bord dorsal 
du tronc maxillaire pour sortir du crâne par le même orifice. La branche ophtalmique superficielle (os) traverse l'alisphénoïde par un trou situé entre ceux de l'optique et du tronc maxillaire et un peu plus haut que ces derniers.

\section{Branches ophtalmiques (Pl. 2, fig. 3, 4, en jaune).}

Nous comprenons sous ce nom un certain nombre de branches nerveuses, formant un système compliqué qu'il n'est pas facile de comparer au nerf ophtalmique décrit chez d'autres espèces par différents auteurs.

La branche la plus élevée sort du plexus ganglionnaire du trijumeau-facial sous forme d'un tronc $(0 s)$, qui est très court. Ce tronc s'engage dans un orifice de l'alisphénoïde et se divise déjà en deux branches durant son passage à travers l'os ou immédiatement après. De la partie supérieure du ganglion sort également une autre branche (opr), presque aussi forte que la première; elle se trouve d'abord accolée au bord externe de celle-ci et lui est inférieure dans le reste de son parcours. Il semble que cette branche n'ait pas toujours été prise en considération ou qu'elle ait été objet d'interprétations différentes. Afin de faciliter l'exposé de la comparaison avec les indications des auteurs il ne sera d'abord question que de la première branche, qui seule semble concorder avec ce que STANNiUs et d'autres appellent branche ophtalmique.

Branche ophtalmique superficielle (fig. 3 et 4, os). Cette branche est divisée en deux rameaux dès sa sortie de la boîte cranienne. Le rameau supérieur (rs), le moins fort, se ramifie peu et doit correspondre à la branche ophtalmique supérieure de Stannius. Le rameau inférieur (ri), branche ophtalmique inférieure de Stannius, a un domaine plus étendu. Très près de sa sortie du tronc, le rameau inférieur émet une ramification, qui semble quelquefois naître directement du tronc et envoie des 
filets nerveux soit dans les enveloppes de l'œil, soit dans les téguments qui l'entourent, soit encore aux orifices nasaux. $\mathrm{Vu}$ - son importance, je la désignerai comme rameau oculo-nasal.

Le rameau supérieur (rs) se dirige en avant, à peu de distance de l'alisphénoïde $(\mathrm{Al})$ et de l'orbitosphénoïde (Ors), qui limitent latéralement le prolongement antérieur de la cavité cranienne. Il s'avance entre l'origine des muscles extenseurs du barbillon et le dilatateur operculaire (fig. 6), et, arrivé devant le bord extérieur de ce dernier, il continue à aller en avant, en s'élevant dans l'épaisseur du frontal principal. Il passe alors près de la paroi médiale de la fosse nasale à laquelle il envoie plusieurs filets. Enfin, il parvient, comme l'indique STannius, sur le prémaxillaire $(\operatorname{Pmx})$; ses ramifications terminales se répandent dans la peau.

Pendant ce trajet, le rameau supérieur a émis plusieurs ramifications. Ce sont, d'abord, avant son passage à travers le frontal, quelques filets peu importants, se perdant dans la graisse qui enveloppe le nerf optique ou dans la peau qui entoure l'œil. Le plus fort de ces filets est émis près de la sortie du crâne et contourne le bord postérieur du dilatateur operculaire, pour se rendre dans la peau, sur le frontal. Pendant qu'il traverse le frontal, le rameau supérieur envoie plusieurs ramifications aux canaux muqueux de la partie antérieure de cet os et à la peau qui le recouvre (fig. 5, rros).

Le rameau inférieur (ri) (branche ophtalmique inférieure de STAnnius), se rend dans la même région que le précédent. D'abord placé juste au-dessous de celui-ci, il s'en écarte un peu pendant le parcours entre le dilatateur operculaire et les extenseurs du barbillon, puis s'en rapproche et le croise par dessous durant le passage à travers le frontal. Il parvient ainsi dans la région médiane du prémaxillaire et se divise à la surface externe de cet os. Les ramifications terminales, dans cette partie, sont moins nombreuses que celles du rameau supérieur et occupent surtout le bord supérieur de la lèvre. 
Non loin de la sortie du crâne, un rameau grêle (Pl. 2, fig. 3, $4 ; n^{\circ} 1$ ) est émis ventralement par le rameau inférieur. Ce rameau va longer le muscle oculaire droit externe, à la surface duquel il est étroitement appliqué. Arrivé à l'extrémité antérieure du muscle, il se bifurque : une partie va dans la peau au voisinage latéral de l'œil, l'autre dans l'enveloppe de l'œil. A peu près au premier tiers de sa longueur, le rameau inférieur envoie encore de son bord latéral un filet mince destiné aussi à l'enveloppe du bulbe oculaire. Enfin se détache un rameau assez fort qui passe sous le frontal et se bifurque. Ses deux filets parviennent à la surface externe de l'os; l'un se rend au bord de l'orifice nasal postérieur, l'autre se prolonge en avant et se ramifie aux environs de l'orifice nasal antérieur. Ce rameau est indiqué dans les fig. 3 et 4 , comme nerf de la narine (Pl. 2, fig. 3, 4, nn).

Il reste à parler du fort rameau oculo-nasal (on) qui se détache du rameau inférieur de la branche ophtalmique superficielle, au moment où celle-ci sort du crâne. Il est d'abord situé un peu au-dessus du rameau ophtalmique inférieur et ne tarde pas à se diviser. Il serait inutile de décrire le trajet de ses diverses ramifications, car elles présentent des variations d'un individu à l'autre ou même entre les deux côtés de la tête chez un même - individu, aussi bien sous le rapport du nombre que de la position.

Ce qui est constant, c'est le fait que la plus forte des ramifications, la plus rapprochée de la ligne médiane, après avoir passé par-dessus le nerf fourni à la narine par le rameau inférieur, longe le bord médial de l'orifice nasal postérieur auquel il envoie de fins filets et se perd dans la peau près de la paroi médiale de la fosse nasale. Cette ramification, donne ellemême un filet très faible allant vers le globe de l'œil (fig. 3, 4, $\mathrm{n}^{0} 2$ ); de plus, elle peut communiquer avec le nerf de la narine (nn) par un filet anastomotique ou bien être fusionnée sur un certain parcours avec un filet provenant du rameau inférieur (ri), comme le montre la figure 4 . 
Les autres ramifications du rameau oculo-nasal sont toujours au moins au nombre de trois. Elles passent au-dessus du nerf optique et se rendent vers le globe oculaire; là, elles s'anastomosent et se répartissent dans la peau autour de l'œil. L'une d'elles (rc) a une destination différente; elle perce la sclérotique près du muscle droit supérieur et se divise en deux filets qui semblent aller tous deux dans l'iris. Cette dernière ramification doit avoir la même fonction que le rameau auquel on a donné le nom de rameau ciliaire. Chez d'autres Poissons, ce rameau est parfois indépendant et provient de la racine du trijumeau, ou bien sort du ganglion de GASSER, ou encore de la branche ophtalmique.

Il est à peine douteux que c'est de quelques ramifications du rameau inférieur (ri) et de son rameau oculo-nasal qu'il s'agit lorsque Stannius dit (p. 37) : « bei Silurus endet der dünne unbeträchtliche, in zwei Aeste gespaltene $R$. ophtalmicus, nach Abgabe der ihm angeschlossen gewesenen Zweige für den Musc. obliquus superior und rectus externus und anderer Zweige für die Stirnhaut, in den Umgebungen der Nasengrube und am aufsteigenden Aste des Zwischenkiefers ». Je n'ai jamais pu voir un des filets de ce rameau pénétrer dans les muscles oculaires nommés. Il en est qui viennent s'accoler à leur face externe o d'une manière très étroite, mais ils ne s'introduisent pas entre leurs fibres : ils se replient généralement en arrière pour se rendre dans les téguments qui environnent l'œil (fig. 5, rrp) ou bien ils percent la sclérotique. D'ailleurs, j'ai trouvé des filets nerveux d'une tout autre origine se rendant aux muscles oculaires. Il est donc permis de supposer que c'est par erreur que Stannius voit dans les rameaux en question des éléments des nerfs moteurs oculaires qui auraient passé dans la voie de

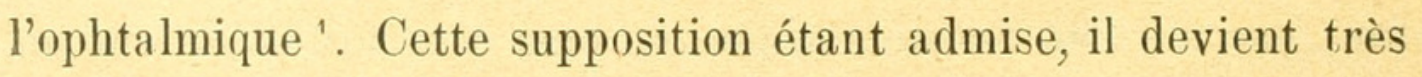

'p. 3ä : a Es ist hervorzuheben, dass bei manchen Fischen Elemente der A ugenmuskelnerven in der Bahn des R. ophthalmicus verlaufen, wie bei Silurus. 
certain que ce sont seulement les deux rameaux de la branche ophtalmique superficielle que l'auteur a considérés comme un nerf ophtalmique à deux branches.

Branche ophtalmique profonde (fig. 3, 4, 6, opr). En sortant du ganglion de Gasser, cette branche importante est appliquée contre la face latérale du petit tronc qui représente la branche ophtalmique superficielle avant sa division en deux rameaux. Il semble, au premier abord, que les deux branches ophtalmiques soient indépendantes l'une de l'autre; on peut, en effet, suivre d'avant en arrière, sur la face dorsale du plexus ganglionnaire, une strie suivant laquelle il est facile de les séparer sur une certaine distance. A partir de la moitié de la longueur du plexus, il est impossible de les isoler complètement l'une de l'autre et du renflement ganglionnaire; mais en arrière de ce point, on voit encore les deux branches réunies former une petite éminence allongée sur le tronc du trijumeaufacial. Cette éminence ressemble donc à un tronc commun qui ne serait pas distinct du plexus ganglionnaire.

Seul, Friant (49) parle d'une branche nerveuse analogue à celle désignée ici comme branche ophtalmique profonde. Il l'a trouvée plus ou moins développée chez les espèces qu'il a étudiées, mais elle serait complètement indépendante; elle aurait son origine propre dans la moelle allongée et ne serait que juxtaposée au trijumeau. Il la désigne comme nerf facial. Sur ce point, Friant diffère de tous ses prédécesseurs qui, eux, regardaient comme facial la branche qui suit dans son trajet le deuxième arc viscéral (CUVIER : branche operculaire du trijumeau; FRIANT: branche mandibulo-hyoïdienne du trijumeau). Voici les raisons qu'il donne : « La branche mandibulo-hyoïdienne du trijumeau a été regardée comme l'analogue du facial par les anatomistes qui n'ont considéré que son trajet à travers l'os temporal et la distribution de ses filets dans les muscles de l'appareil respiratoire. - Ce n'est pas par la simple analogie qu'il fallait la déter- 
miner, c'est par les usages. S'il y a des analogies entre les organes, cela n'est qu'autant qu'il y en a entre leurs fonctions. Le nerf facial des Vertébrés supérieurs a été appelé nerf respiratoire de la tête par Charles BELL, parce qu'il préside à la dilatation des ailes du nez et, par conséquent, au passage de l'air à travers les fosses nasales; mais, chez les Poissons, les fonctions respiratoires s'accomplissent dans des conditions différentes. Les narines sont complètement étrangères à la respiration; cette fonction s'opère par un acte de déglutition de l'eau intimément lié aux mouvements d'ampliation et de resserrement de la cavité buccale. Ce sont les muscles des appareils ptérygomaxillaire et operculaire qui sont ici les agents de la respiration, et ils sont tous animés par le trijumeau. - Le nerf facial présidant à l'expression de la physionomie, et cette expression et ce nerf offrant dans l'échelle animale un développement proportionnel, nous devions, chez les Poissons, rencontrer un facial peu développé. Comme chez les autres Vertébrés, nous trouvons encore placés sous l'influence du facial les muscles qui tiennent sous leur domination l'orifice des narines, seulement ici les constricteurs et les dilatateurs de l'ouverture uasale n'ont plus pour fonction de faire passer le courant d'air affecté aux voies respiratoires, mais seulement de faire parvenir les émanations au nerf olfactif $\gg($ p. 85-86).

Dans les traits essentiels, la description que FrIant donne de son facial, en particulier chez Esox et chez Lota, concorde avec ce qui se voit chez le Silure. Les fibres de cette branche se trouvent bien au-dessus de ce qu'il appelle « racine antérieure du trijumeau » et elle se bifurque, comme chez la Lotte, un peu après la sortie du crâne.

Le rameau supérieur (fig. $4, \mathrm{n}^{\circ} 4$ ) est aussi le moins fort. Il se trouve au-dessus du muscle droit supérieur (dans la figure, il est un peu déplacé vers la gauche) et se partage en deux parties inégales : la plus grêle, l'interne, s'unit à un filet provenant du 
rameau inférieur de la branche ophtalmique superficielle, et le filet ainsi constitué se replie en arrière et vient se terminer dans le tégument, près de l'orifice nasal postérieur; l'autre partie, plus forte, envoie quelques filets à l'enveloppe de l'œil, puis s'infléchit aussi un peu en arrière et se ramifie dans la peau à la face latérale de la tête.

Le rameau inférieur semble être la continuation de la branche qui est sortie du crâne au-dessus du tronc maxillaire commun et par le même orifice. Il chemine appliqué étroitement à la branche maxillaire supérieure et envoie dorsalement un filet dans l'enveloppe de l'œil, à la partie inférieure de celui-ci (fig. 3, $\left.\mathrm{n}^{\circ} 4\right)$.

Du bord dorsal du nerf maxillaire supérieur se détache un cordon assez fort qui se fusionne avec le rameau inférieur de l'ophtalmique profond. A part cette communication, le maxillaire supérieur et la branche de l'ophtalmique profond, quoique unis par du tissu conjonctif, restent distincts l'un de l'autre. Les deux branches superposées passent contre la face externe de l'extenseur inférieur du barbillon (fig. 6) et, descendant un peu, se trouvent à la limite inférieure de ce muscle et à la limite supérieure du muscle rétracteur. C'est alors que les deux branches se séparent, le maxillaire supérieur descend en s'appliquant contre la face interne du muscle rétracteur, tandis que la branche de l'ophtalmique passe au-dessus des muscles extenseurs du barbillon, en croisant les muscles oculaires obliques au-dessous de leur origine.

Cette dernière branche se divise déjà en cinq ou six ramifications qui restent unies jusqu'au niveau de l'orifice nasal postérieur; à partir de là, elles s'étalent en éventail, les unes vont au pourtour de la fosse nasale, d'autres vers l'orifice nasal antérieur et sur l'os prémaxillaire. L'une d'elles se dirige vers la base du barbillon auquel elle envoie un filet peaucier très court, s'infléchit latéralement en passant devant l'os maxillaire 
et va jusqu'à l'angle de la bouche. Enfin, une autre ramification assez forte s'enfonce dans l'os prémaxillaire et s'infléchit médialement. Elle se trouve alors à la base des dents qui garnissent la face inférieure de cet os et se résout en plusieurs filets, dont l'un s'anastomose avec l'un des rameaux terminaux du nerf palatin. Par sa distribution et son anastomose avec le nerf palatin, cette ramification laisse supposer qu'elle est une dérivation du nerf maxillaire supérieur. En effet, si l'on suit vers la périphérie les éléments du cordon qui a passé du nerf maxillaire supérieur dans la branche ophtalmique profonde, on voit qu'ils restent à la face inférieure de cette dernière et qu'ils passent dans la ramification destinée à la face alvéolaire du prémaxillaire. Malgré l'apparence, cette ramification doit donc être attribuée à la branche maxillaire supérieure.

La branche qui vient d'être décrite comme ophtalmique profond semble donc correspondre au facial de Friant. Mais la dénomination de facial est à rejeter puisque cette branche, forme, avec la branche ophtalmique superficielle, un tronc commun, non séparé du plexus et alimenté par des éléments des portions sous-jacentes du plexus ganglionnaire (v. p. 55). En outre, dans cette comparaison des Poissons avec les Vertébrés supérieurs, Friant met en parallèle les faibles muscles peauciers de la narine des uns avec toute la musculature qui préside à l'expression de la physionomie chez les autres, sans tenir compte de la provenance de cette dernière ${ }^{~}$. On peut cependant dire que l'auteur a eu partiellement raison si, avec GoroNowitsch, on admet que l'ophtalmique profond contient des éléments du facial segmental; ce seraient alors ces éléments qui actionneraient les muscles peauciers de la narine. Mais, ceci ne justifie nullement l'idée de regarder cette branche comme repré-

${ }^{1}$ Ruge (102, p. 204) : "So lange eine mimische Muskulatur besteht, geht auch der Facialis zu ihr. Derselbe war ihr niemals fremd; wohl hatte sie als motorisches Facialis-Endgebiet ursprünglich mit dem Gesichte nichts zu thun •. 
RECHERCHES SUR LE SILURUS GLANIS.

sentant exclusivement le facial, puisque d'autres branches du complexe reçoivent aussi des éléments du facial segmental.

On pourrait être tenté de comparer la branche en question au nerf rostral du trijumeau postérieur de la Lotte (GoRoNoWITSCH). Celui-ci est intimément réuni sur un certain trajet au nerf maxillaire supérieur (homologue du nerf rostral du trijumeau antérieur de Acipenser); il s'en détache distalement pour se rendre aux os antérieurs de la série sous-orbitaire et aux canaux muqueux de cette région. Une telle comparaison doit être immédiatement abandonnée, parce que cette branche aurait, chez le Silure, un développement hors de proportion avec l'état des os sous-orbitaires. En outre, son domaine d'innervation est beaucoup plus étendu que ne l'indiquent les observations de Gononowitsch; elle semble partager celui de la branche décrite comme branche ophtalmique superficielle.

Il paraît donc, aussi bien d'après l'origine que d'après la distribution, qu'il soit juste de regarder cette branche comme un ophtalmique profond.

En résumé, l'ophtalmique du Silure se compose de deux branches importantes dont la superficielle est divisée dès la sortie du crâne en deux forts rameaux. Il est à remarquer que ces trois parties principales sont toujours parfaitement distinctes les unes des autres et n'ont de communications entre elles que par leurs ramifications. Mais, l'éloignement des deux branches et la grande extension de leurs rameaux terminaux apparaissent comme une conséquence toute naturelle si l'on remarque que l'étendue considérable de la tête du Silure dans le sens latéral correspond surtout à un fort développement des pièces squelettiques de la région médiane.

\section{Branches maxillaires (PI. 2, fig. 3, 4, en bleu).}

Les branches maxillaires, chez le Silure, sortent de la partie antérieure et inférieure du plexus du trijumeau-facial par un 
tronc commun court, mais très volumineux. Celui-ci quitte le crâne neural par un orifice situé dans la partie antérieure du pétreux, près de la limite de cet os et de l'alisphénoïde. L'orifice est ordinairement simple du còté interne, tandis que du côté externe, il est toujours divisé en deux parties par une petite lamelle horizontale, conjonctive, quelquefois ossifiée, laquelle partage le tronc maxillaire en deux portions superposées.

La division du tronc maxillaire se continue en arrière et en avant de l'orifice de sortie; elle est, du reste, rendue visible, surtout sur la face médiale du tronc, par une strie longitudinale souvent très accentuée. En avant, la séparation des deux parties n'aboutit jamais, comme on pourrait d'abord le penser, à la bifurcation du tronc en branches maxillaire supérieure et maxillaire inférieure, mais se prolonge sur les deux branches maxillaires. Tant que les deux branches maxillaires restent rapprochées, elles ne sont exactement ni superposées, ni juxtaposées, mais la branche inférieure est située un peu plus en dehors (latéralement) que l'autre. Ainsi, le plan de séparation des deux portions, d'horizontal qu'il était dans le tronc commun, devient oblique de haut en bas et de dedans en dehors, et le plan de bifurcation lui reste perpendiculaire (v. le schéma, p. 68). En arrière de la lamelle osseuse, on ne peut séparer les deux parties que sur une faible distance, car on arrive bientôt dans la région du plexus où les nombreux échanges de fibres rendent impossible la délimitation exacte des portions.

On peut donc dire que cette sorte de boutonnière s'étend sur le tronc maxillaire commun, sur la partie antérieure du ganglion de Gasser et sur les deux branches maxillaires. Sur ces dernières, elle s'avance assez loin et, il est vrai, davantage sur la branche inférieure que sur la supérieure. Les deux couches sont intimement accolées, sauf au passage de la lamelle osseuse, et sont unies par du tissu conjonctif. Bien que la séparation exacte 
ne puisse pas être effectuée aussi loin en arrière, il est facile de voir que la deuxième portion du plexus (v. p. 55) forme principalement la partie du tronc maxillaire située au-dessous de la lamelle osseuse et que la troisième portion va surtout dans la partie supérieure du tronc.

D'après les connexions de ces portions du plexus avec les autres portions, on peut, en admettant que le trijumeau-facial est formé de trois nerfs segmentaux, évaluer approximativement la part prise par chacun d'eux à la formation des deux parties du tronc maxillaire. La deuxième portion, qui sort de la moelle allongée inférieurement et antérieurement, doit contenir surtout des fibres du trijumeau antérieur (I); quand à la troisième portion, la plus forte, elle doit contenir la majorité des éléments du trijumeau postérieur (II). Cette troisième portion est en relation très étroite avec celle qui va principalement dans le tronc hyoïdeo-mandibulaire, et il se fait entre elles un échange réciproque de fibres. Elle doit donc contenir, à côté des éléments du trijumeau postérieur, des éléments du facial; mais ces deux sortes d'éléments ne peuvent être distinguées. Tout ce qu'on peut dire, c'est que la partie faciale de la portion se trouve du côté latéral et ne peut aller que dans la branche maxillaire inférieure.

Il résulte de tout cela que le maxillaire supérieur est formé d'une couche inférieure provenant du trijumeau antérieur et d'une couche supérieure fournie par le trijumeau postérieur; le maxillaire inférieur est constitué inférieurement par le trijumeau antérieur et supérieurement par le facial.

Les figures schématiques ci-dessous peuvent donner une idée de la disposition des élémeuts dans le tronc maxillaire et de leur répartition dans les deux branches, mais elles ne tiennent pas compte des relations existant d'une part entre les deux portions du plexus qui alimentent le tronc maxillaire et, d'autre part, entre ces deux portions et les autres. Par exemple, toutes les 
fibres du trijumeau postérieur ne vont pas dans le maxillaire supérieur, une bonne partie de celles-ci se rendent dans la portion du plexus d'où dérive l'ophtalmique.
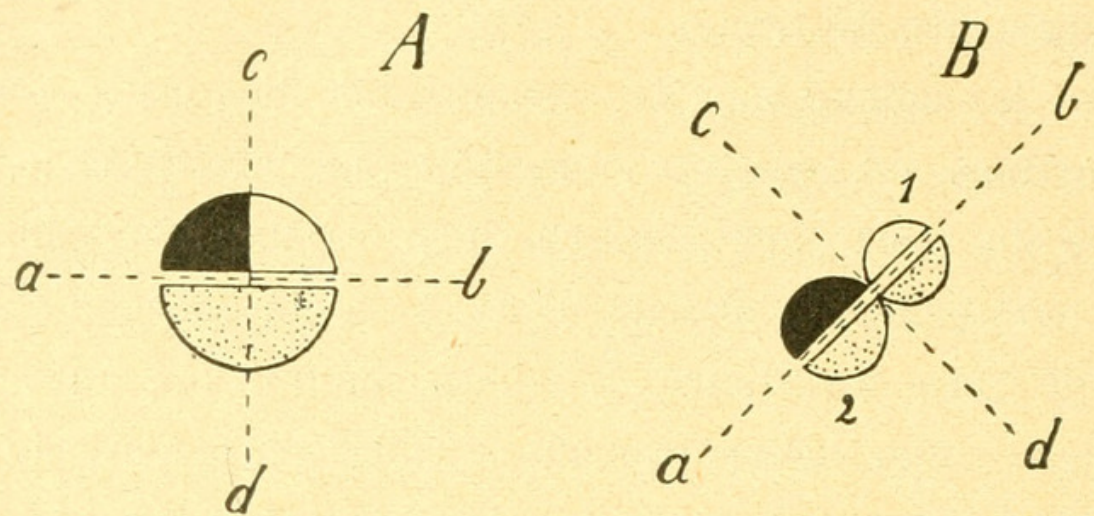

Sections transversales schématiques, $A$, du tronc maxillaire commun de gauche, $B$, des branches maxillaire supérieure (1) et infêrieure (2) à leur sortie de ce dernier. $a b$, plan de séparation en denx portions superposées.

$c d$, plan suivant lequel le tronc commun se divise en deux branches.

Partie ponctuée $=$ éléments du trijumeau segmental antérieur (trij. I) .

Partie blanche $=$ éléments du trijumean segmental postérieur (trij. II) .

Partie noire $=$ éléments du facial segmental.

Il semble que l'on puisse expliquer ainsi la présence de ces portions qui sont parfois si nettement délimitées, et particulièrement cette division très constante du tronc maxillaire et des deux branches qui en sortent, en deux portions superposées. Ces observations acquièrent une plus grande valeur si on les compare aux résultats beaucoup plus précis que GoRoNowITsch a obtenus par des coupes en séries (v.p. 53 du présent travail). D'après cet auteur, le maxillaire supérieur de la Lotte est constitué également par des éléments du trijumeau antérieur et du trijumeau postérieur, le maxillaire inférieur par des éléments du trijumeau antérieur et du facial. Je dois cependant ajouter qu'il paraîtrait étonnant que, chez le Silure, une partie des fibres du trijumeau postérieur ne soient pas réunies à celles du facial pour former le nerf maxillaire inférieur.

Avant de se diviser en deux branches, le tronc maxillaire commun donne naissance à plusieurs rameaux destinés à la 
muqueuse buccale, à la musculature de la mâchoire, de l'arc palatin et du barbillon. Ce sont les suivants :

$1^{\circ}$ Rameau de la muqueuse buccale. Ce rameau sort, en réalité, de la face inférieure du ganglion de Gasser, mais il peut être compté au nombre des rameaux du tronc maxillaire, car il provient de la portion ganglionnaire qui fournit la partie inférieure de ce tronc. Recouvert par ce dernier, il se dirige également en avant et sort du crâne avec lui ou un peu au-dessous. Tantôt il va encore dans la même direction en passant entre l'os pétreux et le muscle adducteur hyomandibulaire, se replie en arrière à la face inférieure du muscle pour arriver un peu en avant du tronc hyoïdeo-mandibulaire, tantôt il arrive directement à la même place en suivant le bord postérieur du même muscle. Il se ramifie dans la muqueuse de la région postérieure et latérale de la cavité buccale.

$2^{\circ}$ Rameau du muscle élévateur de l'arc palatin et du muscle dilatateur operculaire. Il est émis par le tronc maxillaire durant son passage dans le canal osseux, et en sort avec lui, accolé à son bord latéral. Son origine se trouve dans la portion inférieure du tronc; il est donc probable qu'il dépend du trijumeau antérieur. Il va en avant en passant près du bord supérieur de la portion profonde du muscle adducteur mandibulaire et, s'élevant un peu, il atteint la face inférieure du muscle élévateur de l'arc palatin. Il se ramifie dans ce muscle après avoir donné une ou deux ramifications qui s'avancent médialement et pénètrent dans le muscle dilatateur operculaire, non loin de son origine.

$3^{\circ}$ Rameau de l'adducteur mandibulaire et du rétracteur du barbillon. Ce rameau est beaucoup plus fort que les deux précédents. Il parait sortir de la face latérale de la branche maxillaire inférieure, près de sa naissance, mais, en réalité, il arrive jusqu'à ce point en étant appliqué contre le tronc maxillaire, puis contre le maxillaire inférieur. Il sort du bord inférieur et 
latéral du tronc au moment où celui-ci quitte le canal de l'os pétreux. Son origine est donc analogue à celle du rameau précédent. Le rameau se dirige latéralement, pénètre dans la portion profonde de l'adducteur mandibulaire, traverse obliquement la masse de ce muscle en lui abandonnant quelques filets, et en sort à peu près au centre de la face externe. Il se trouve alors devant le bord antérieur de l'élévateur palatin et se replie en arrière et en haut pour entrer dans la portion superficielle de l'adducteur mandibulaire. Ce rameau se divise beaucoup et envoie ses filets terminaux dans la masse musculaire de la mâchoire (fig. 3, 4, 5, 6, ram).

Enfin, le rameau adducteur mandibulaire émet un nerf assez fort (fig. 3, 4, 6, rrb), destiné au muscle rétracteur du barbillon. Sur un même exemplaire, nous avons observé que, du côté droit de la tête, ce nerf se séparait du rameau de l'adducteur mandibulaire très près de son origine, tandis que, du côté gauche, il s'en séparait plus distalement, c'est-à-dire seulement à l'endroit où le rameau va pénétrer dans la portion profonde de l'adducteur mandibulaire. Dans les deux cas, le nerf chemine vers l'avant à la face externe du métaptérygoïde et entre dans la partie postérieure et supérieure du muscle rétracteur.

Stannius $\left(16\right.$, p. $\left.41,5^{\circ}\right)$ dit que le muscle rétracteur du barbillon est innervé par un rameau venant directement du tronc maxillaire. Il est probable que l'origine de ce nerf, que j'ai toujours constatée sur le rameau adducteur mandibulaire, mais à des places variables, peut être reculée à tel point qu'elle se trouve sur le tronc commun lui-même, un peu en arrière du rameau adducteur mandibulaire. Quant au rameau venant également du tronc commun et allant au muscle « qui tire le barbillon vers l'intérieur », nous ne l'avons trouvé sur aucun exemplaire malgré d'attentives recherches. Par contre, il y avait toujours, pour les deux muscles superposés qui accomplis- 
sent cette fonction, un rameau du tronc hyoïdeo-mandibulaire dont il sera question plus tard (v. p. 82).

$4^{\circ}$ Rameau sphéno-palatin (Pl. 2, fig. 4, sp). Il correspond au nerf ptérygo-palatin de CuvIER et au nerf palatin de STANNIUs; son trajet et sa distribution justifient parfaitement sa désignation comme sphéno-palatin (FRIANT, 49).

Stannius $(16$, p. 55$)$ a vu le nerf palatin de la plupart des Poissons osseux sortir du plexus ganglionnaire et montrer une certaine indépendance vis-à-vis du trijumeau et du facial; chez Silurus glanis, il se détache du tronc maxillaire commun et n'a aucune communication avec le facial; enfin chez Chimaera et Raja, il sort du facial.

Friant $(49$, p. 75-80) a trouvé, sur neuf espèces de Poissons qu'il a examinées, huit espèces oủ le sphéno-palatin sortait de la partie postérieure, inférieure et interne du ganglion du trijumeau, et une seule (Perca fluviatilis) où il naissait aussi bien du tronc mandibulo-hyoïdien (facial de Stannius) que du ganglion du trijumeau.

Gegenbaur (35, p. 281) a trouvé chez les Sélaciens un nerf palatin venant du facial, et représentant le rameau pharyngien d'un nerf homologue d'un nerf spinal. Chez les Poissons osseux (31, p. 701), le nerf palatin dépend du trijumeau, mais il a des rapports avec la branche représentant le facial. Cette dernière indication ne se retrouve pas dans l'ouvrage paru tout récemment sous le titre de: Vergleichende Anatomie der Wirbelthiere; il y est dit simplement $(103$, p. 810$)$ que le nerf palatin est un rameau du nerf facial et qu'il peut posséder une racine indépendante ayant son propre ganglion.

Goronowitsch (100, p. 27), a montré que chez la Lotte, le rameau palatin qui sort apparemment de la face ventrale du plexus a son origine dans le facial. Ce fait est d'une grande importance pour la théorie de la segmentation de la tête des Vertébrés, car le palatin des Poissons osseux doit représenter le rameau pharyngien du facial segmental. 
Ruge (102, p. 206), pense aussi que le rameau palatin doit toujours naître du facial, même lorsqu'il est plus ou moins étroitement accolé au tronc du trijumeau. Cet auteur ne nous dit pas si c'est par des recherches spéciales qu'il est arrivé à cette conviction.

Chez le Silure, le rameau se détache du tronc maxillaire à son bord médial et inférieur, ordinairement au moment où le tronc vient de traverser la paroi cranienne. En essayant d'isoler ses éléments en arrière, je ne suis jamais parvenu à les voir s'éloigner de ceux qui représentent très probablement le trijumeau antérieur. Mais ce procédé est trop primitif pour m'autoriser à penser qu'il ne puisse pas exister d'autre origine.

Une particularité que je n'ai pu constater exactement qu'une seule fois est que le nerf sphéno-palatin émet, à une petite distance de sa naissance, un filet grêle qui s'unit sur un long parcours à un filet du rameau adducteur palatin, qui dépend luimême du tronc hyoïdeo-mandibulaire. Ce fait semblerait indiquer que le nerf palatin dépend du nerf facial, mais malheureusement, il ne s'agit pas là d'une vraie anastomose; le filet du sphéno-palatin se sépare distalement et traverse l'os palatin pour se perdre dans la muqueuse.

Dans le reste de son trajet, le nerf (fig. 6) suit le bord latéral du parasphénoïde en passant entre l'origine de l'extenseur inférieur du barbillon et celle de l'adducteur palatin, puis il s'enfonce dans le vomer et dans le prémaxillaire en s'approchant de leur face inférieure. Pendant son passage sur le premier de ces os, il émet un filet latéral et un filet médial qui vont dans la muqueuse alvéolaire de cette région ; puis, sur le prémaxillaire, il forme deux filets terminaux, l'un latéral, l'autre médial. Ce dernier s'unit à un filet provenant du rameau du maxillaire supérieur momentanément accolé à la branche ophtalmique profonde (v. p. 61). Le rameau ainsi constitué se rend, de même que le rameau médial, dans la muqueuse alvéolaire du pré- 
maxillaire; une seule de ses ramifications s'élève un peu et suit le bord de la lèvre où elle se perd dans la peau.

Nerf maxillaire supérieur (Pl. 2, fig. 3 et 4 , mxs).

Le tronc maxillaire commun, après avoir émis les ramifications qui viennent d'être mentionnées, se divise en deux branches d'inégal volume. La branche supérieure est la moins forte; elle se dirige presque horizontalement en avant et un peu obliquement vers l'extérieur. La branche profonde de l'ophtalmique (opr) vient s'appliquer contre son bord supérieur et passe avec elle sous le nerf optique, puis au-dessous de l'origine des deux muscles oculaires obliques (fig. 6). Les deux branches s'éloignent l'une de l'autre après s'être engagées entre les muscles extenseur et rétracteur du barbillon. Le nerf maxillaire supérieur descend un peu et longe la face inférieure du muscle rétracteur; il pénètre ensuite dans la base du barbillon et le suit jusqu'à l'extrémité. Le barbillon est soutenu, dans toute sa longueur, par un axe cartilagineux, aplati dans le sens dorsoventral. Le nerf est situé entre la peau et l'axe cartilagineux, le long de l'arête antérieure de ce dernier, et envoie de nombreux filets à toute la surface de l'appendice tactile.

A quelque distance de son passage sous le nerf optique, la branche maxillaire supérieure émet un cordon assez fort, qui s'unit bientôt à la branche profonde de l'ophtalmique. Comme il a été dit précédemment (p. 64), il n'y a pas là une anastomose proprement dite, quoique distalement toute trace extérieure de séparation ait disparu.

En effet, si on isole les éléments venant du maxillaire supérieur, de ceux de l'ophtalmique profond, on arrive à une strie latérale de plus en plus nette qui aboutit à la séparation des deux parties. La partie maxillaire se rend à la face inférieure 
du prémaxillaire (Pmx), dans la muqueuse alvéolaire qui recouvre cet $o$; on peut donc l'appeler rameau prémaxillaire $(\mathrm{pmx}$, fig. 3 et 4). L'un de ses filets s'anastomose toujours avec l'un des rameaux terminaux du nerf sphéno-palatin.

Le nerf maxillaire supérieur du Silure a donc ceci de remarquable, que la plus forte partie de ses éléments constitue le nerf sensible du barbillon, et qu'un contingent beaucoup moins fort se rend à la face inférieure du prémaxillaire, où sa fonction est également purement sensorielle.

D'après cela, le nerf ne recevrait des racines du trijumeau antérieur et du trijumeau postérieur que des fibres sensibles, et ne serait pas un homologue parfait du maxillaire supérieur de la Lotte, lequel contient des éléments destinés au muscle adducteur mandibulaire. De plus, la portion venant du trijumeau postérieur ne se détache pas, comme chez ce dernier Poisson, pour aller innerver les canaux muqueux des os sous-orbitaires, mais pénètre dans le barbillon avec la majeure partie de celle qui provient du trijumeau antérieur.

Nerf maxillaire inférieur (Pl. 2, fig. 3 et 4, mxi).

En sortant du tronc maxillaire commun, la branche maxillaire inférieure est formée des deux parties superposées dont il a déjà été question plus haut (p. 66). Ces deux parties peuvent être facilement isolées l'une de l'autre sur une assez grande longueur, mais elles finissent par se fusionner si intimement qu'il est difficile d'indiquer exactement quelle part chacune d'elles prend à la formation des diverses ramifications du maxillaire inférieur.

Le nerf suit d'abord la branche maxillaire supérieure contre son bord latéral inférieur, puis s'en éloigne bientôt en se dirigeant plus latéralement et en bas. Il passe ainsi obliquement 
sur l'arc ptérygo-palatin et sous la portion profonde du muscle adducteur mandibulaire. Durant cette partie de son trajet, il est inférieur et parallèle au nerf optique.

Des rameaux destinés à la musculature de la mâchoire, de l'arc palatin, du barbillon et de l'opercule sont livrés par le tronc maxillaire commun et ont été décrits précédemment. D'autres rameaux moins importants dépendent directement du nerf maxillaire inférieur. Une première ramification sort dorsalement du nerf qu'elle accompagne jusqu'au-dessous du globe oculaire (fig. 3). Là, elle se bifurque; une partie va en avant et se perd dans la peau située au-devant de l'œil, l'autre se replie en arrière et va assez loin à la périphérie du muscle adducteur mandibulaire (fig. 5, rrp). Avant de passer au-dessous du globe oculaire, le maxillaire inférieur émet encore dorsalement un rameau mince, qui traverse la membrane reliant l'anneau sousorbitaire au plafond de la cavité buccale et entre dans le revêtement cutané du barbillon, où il s'étend le long de l'arête postérieure, sur une certaine longueur; c'est le nerf postérieur du barbillon (fig. 3 et $4, \mathrm{npb}$ ).

Ensuite, la branche maxillaire inférieure s'infléchit vers l'extérieur et en bas, en passant devant le bord antérieur et supérieur de la portion profonde du muscle adducteur mandibulaire. En ce point, elle est déjà divisée en deux parties qui restent superposées et accolées jusqu'à ce qu'elles arrivent sur la masse tendineuse de la portion superficielle du muscle adducteur mandibulaire. Là, un peu en arrière de l'angle de la bouche, ces deux parties se séparent. La supérieure contourne extérieurement la masse tendineuse et se place contre la face externe de l'articulaire où elle est recouverte par le fascia du muscle adducteur mandibulaire; l'autre partie s'infléchit vers l'intérieur et en avant, en passant entre les deux portions que forme la masse tendineuse de l'adducteur mandibulaire et arrive à la face interne de l'articulaire. La première constitue la branche man- 
dibulaire externe (fig. $3,4,5$ et 9 , me), la deuxième la branche mandibulaire interne (mi).

La branche mandibulaire externe, pendant son passage sur l'articulaire, envoie plusieurs filets dans la peau de cette région. Ensuite, elle se prolonge à la face externe du dental, au-dessus de la branche mandibulaire du tronc hyoïdeo-mandibulaire. Ces deux branches cheminent ainsi, très rapprochées, sans se fusionner, mais réunies simplement par plusieurs filets anastomotiques, puis, arrivées dans la partie moyenne de la mandibule, elles s'éloignent l'ume de l'autre. La branche du maxillaire inférieur s'élève un peu et se bifurque. L'un des filets vient suivre le bord de la lèvre, je l'appellerai rameau labial (rl); l'autre se trouve plus en arrière et se ramifie dans la muqueuse traversée par les nombreuses petites dents, c'est le rameau alvéolaire antérieur (fig. 4 et 9, ra ${ }_{1}$ ). Ces deux rameaux terminaux vont jusqu'à la symphyse de la mâchoire inférieure. Le premier contracte plusieurs unions avec des filets de la branche mandibulaire du tronc hyoïdeo-mandibulaire.

La branche mandibulaire interne est plus importante par son volume et le nombre de ses ramifications. Elle passe entre l'articulaire et la partie de la portion profonde du muscle adducteur mandibulaire qui s'insère à la face interne du dental et sur le cartilage de MEckEL. Elle émet alors un rameau alvéolaire (fig. $3,4,9, \mathrm{ra}_{2}$ ) qui longe le bord supérieur et interne du cartilage de MEckel en se plaçant sur le prolongement du muscle adducteur mandibulaire, puis s'élève à la face interne de l'os dental. Ce rameau se termine par deux filets destinés à la muqueuse du dental et à la lamelle qui s'étend un peu en arrière; il a donc la même fonction que le rameau alvéolaire de la branche externe, mais il est situé postérieurement par rapport à celui-ci.

La branche continuant à aller obliquement en avant et en bas, entre dans la rainure du dental qui loge le cartilage de MEckel, circule autour de ce dernier et se bifurque avant 
de réapparaître au bord inférieur de la rainure. Les deux rameaux ainsi formés descendent sur la face interne du dental, l'un dirigé en avant, l'autre en arrière, et viennent émerger à la face inférieure de la mandibule, à la limite du muscle géniohyoïdien (fig. 9).

Le rameau antérieur s'avance à la face inférieure de ce muscle et ne tarde pas à se trifurquer. La plus forte ramification (a) entre dans le barbillon antérieur (Ba) dont elle suit l'axe cartilagineux jusqu'à l'extrémité. Une autre (b), va médialement jusqu'à la symphyse de la mandibule et se rend en partie dans le muscle intermandibulaire $(\operatorname{Im} d)$, en partie dans la peau environnante où ses filets entrent fréquemment en connexion avec ceux de la branche mandibulaire externe et de la branche mandibulaire du tronc hyoïdeo-mandibulaire. Enfin, la troisième ramification (c) va en arrière et s'enfonce dans le muscle génio-hyoïdien, de même que d'autres rameaux moins importants dépendant des deux ramifications précédentes.

Le rameau postérieur suit aussi la face inférieure de ce muscle; il fournit le nerf (d) du barbillon postérieur (Bp), se prolonge en arrière (e) jusqu'à la base des rayons branchiostèges, puis se replie en avant et médialement. Ses ramifications nombreuses et très étendues se distribuent toutes dans la peau qui revêt le muscle génio-hyoïdien et la membrane branchiostège.

Les deux branches du maxillaire inférieur du Silure, comparées avec celles des autres Poissons, présentent quelques particularités de détail sur lesquelles il serait inutile d'insister. Il suffira de faire ressortir ici deux points importants.

En premier lieu, la force et l'extension de la branche externe, qui n'est généralement représentée chez les autres Poissons osseux que par une faible ramification plus ou moins étendue et n'est alors regardée que comme un filet latéral du nerf maxillaire inférieur (v. Friant, p. 41 et suiv.). Cette branche est puissamment renforcée dans son action par une branche à peu 
près de même volume, venue du tronc hyoïdeo-mandibulaire, et qui doit manquer aux autres Téléostéens (v. StanniUs, Friant, Goronowitsch). Une si riche innervation du tégument de la mandibule s'explique facilement comme adaptation au genre de vie du Silure. En effet, celui-ci ne peut être que faiblement secouru dans la recherche de sa nourriture par son organe visuel, fort peu développé. Comme compensation à cette incapacité de l'œil, nous voyons le sens tactile devenir plus intense; les deux branches en question étalent leurs nombreuses ramifications à la surface de la mandibule, laquelle est d'une largeur considérable. Cette explication devient plus plausible si l'on prend en considération le vaste réseau peaucier formé par l'ophtalmique sur la face frontale (voir fig. 5 ), le fort développement des barbillons et, enfin, la présence d'une branche mandibulaire externe du maxillaire inférieur, chez la Lotte, qui chasse ordinairement sur le fond des lacs.

Le deuxième point concerne l'innervation des muscles intermandibulaire et génio-hyoüdien par la branche interne. B. Vetter $(48$, p. 513. Pl. XIII, fig. 8) a indiqué que chez le Brochet, cette branche, après avoir émis un rameau analogue au rameau alvéolaire postérieur $\left(\mathrm{ra}_{2}\right)$, se fusionnerait avec une branche du facial (notre branche mandibulaire interne du tronc hyoïdeo-mandibulaire) et que le nerf ainsi constitué irait dans les muscles intermandibulaire et génio-hyoïdien, ainsi que dans la peau. L'auteur suppose cependant que les filets moteurs doivent provenir seulement du maxillaire inférieur, car le muscle intermandibulaire et la portion du génio-hyoïdien innervée par eux doivent appartenir au premier arc viscéral. Chez le Silure, les deux muscles ne sont sûrement innervés que par la branche venant du maxillaire inférieur, parce que la branche mandibulaire interne $d u$ tronc hyoïdeo-mandibulaire ne fait que la croiser, sans entrer en communication avec elle. Il est probable qu'il en est de même chez le Brochet, car 
Friant $(49)^{\wedge}$ nous donne sur ce Poisson des renseignements qui concordent avec ce que j’ai observé chez le Silure.

Une raison plus forte peut pourtant faire admettre que les muscles en question reçoivent par la voie du maxillaire inférieur des éléments du trijumeau et du facial : c'est la composition du maxillaire inférieur par ces deux derniers (p. 67). Mais, malgré l’impossibilité de suivre les deux sortes d'éléments jusque dans les dernières ramifications, il est à peine douteux que les fibres du facial, par le fait qu'elles occupent dans le tronc du maxillaire inférieur la région dorso-latérale, doivent, avant tout, passer dans les deux rameaux dorsaux du tronc et dans la branche mandibulaire externe. Ces éléments du facial seraient donc tous sensibles; ceux qui pourraient passer dans la branche interne se rendraient également dans la peau, et le muscle intermandibulaire ainsi que le génio-hyoïdien seraient innervés exclusivement par le trijumeau antérieur.

3. Branche hyoüdeo-mandibulaire ou facial. (Pl. 2, 3, fig. 3, 4, 5, 6, hmd; fig. 3 et 4 , en vert).

Comme nous l'avons déjà dit, le tronc hyoïdeo-mandibulaire des Poissons osseux a reçu des appellations fort différentes et a été regardé tantôt comme un simple rameau du trijumeau (Cuvier et Valenciennes, Friant), tantôt comme un facial dépendant plus ou moins du trijumeau (Stannius, Baudelot, GEgenbaur). En réalité, c'est, au même titre que les branches ophtalmiques et maxillaires, une branche du complexe trijumeau-facial, car il ne contient pas uniquement des éléments du

${ }^{1}$ P. 42 : « Le rameau inférieur terminal du maxillaire inférieur, descend d'abord obliquement le long de la face interne de la mâchoire, se porle sous le cartilage de Mєскец, puis se dirigeant presque horizontalement en avant et en dedans, se place au-dessus du nerf mandibulaire (du tronc hyoïdeo-mandibulaire) au bord supérieur duquel il s'accole, puis le croise.... 
facial et tous les éléments du facial n'entrent pas dans sa voie. Nous allons d'abord chercher de quelle partie du plexus ganglionnaire la branche provient et reconnaitre si celle-ci est indépendante ou non des autres parties.

La branche hyoüdeo-mandibulaire sort latéralement et plutôt ventralement du ganglion de GASSER. Elle tire principalement son origine de la partie du plexus que j'ai désignée comme première portion (p. 55), soit celle qui naît de la moelle allongée le plus en arrière; dans la masse ganglionnaire, elle est située ventro-latéralement par rapport aux autres. Sans exclure la possibilité d'échanges d'éléments dans l'intérieur de l'encéphale, on peut donc admettre que cette portion contient la majeure partie des éléments du facial. En tout cas, elle renferme toute la racine ventrale du facial, car celle-ci peut être isolée de la portion dès la sortie du cerveau. Ainsi, le reste de la portion est surtout formé par la racine dorsale du facial; il présente, du reste, un renflement très sensible.

La première portion du plexus est en relation très étroite avec la troisième (v. p. 67); immédiatement après la sortie de la moelle allongée commence un échange réciproque de fibres. La première portion envoie d'abord un fort contingent de fibres à la troisième, puis elle reçoit de cette dernière une quantité moins considérable d'éléments du trijumeau postérieur, sous la forme d'un cordon court, mais assez facilement visible. Je n'ai jamais observé de passage direct d'éléments entre la première et la deuxième portion.

Goronowitsch (100 p. 28) dit que le tronc hyoïdeo-mandibulaire de la Lotte, est formé d'éléments des trois nerfs segmentaux du complexe. Il se pourrait qu'il en fût de même chez le Silure, mais, pour s'en assurer, il serait absolument nécessaire de connaitre très exactement l'origine centrale de tous les composants de chaque portion du plexus ainsi que leurs relations à l'intérieur de la masse cérébrale, recherche que je ne puis 
entreprendre. Nous savons par Stannius $(16$, p. 25) que chez le Silure de tels échanges intra-cérébraux doivent avoir lieu. Par conséquent, les portions plus ou moins bien délimitées que l'on reconnait dans le plexus ne représentent certainement pas des troncs de nerfs segmentaux. Il est donc bien possible que des éléments du trijumeau antérieur se trouvent déjà dans la première portion, avant qu'elle sorte du cerveau ou encore qu'elle en reçoive par la voie du cordon qui lui vient de la troisième portion.

Ce que l'on peut affirmer, c'est que le tronc hyoïdeo-mandibulaire comprend une grande partie du facial, mais qu'il reçoit aussi une certaine partie de ses éléments du trijumeau.

Le tronc en question ne peut donc être mieux désigné que comme branche hyoïdeo-mandibulaire du trijumeau-facial ${ }^{~}{ }^{1} . \mathrm{Si}$, pour ne pas compliquer la nomenclature, on laisse subsister le terme de facial, il faut se souvenir que l'homonymie des nerfs chez les Téléostéens et les Sélaciens par exemple, n’implique nullement l'idée d'homologie.

Stannius (16 p. 47) a montré que chez la plupart des Téléostéens, le tronc hyoïdeo-mandibulaire est renforcé par une branche courte, issue du plexus du trijumeau et sortant de la cavité cranienne avec le trone maxillaire. Cette branche qu'il appelle rameau communicant du trijumeau au facial manquerait chez Silurus ainsi que chez les Poissons où le facial ne sort·pas du crâne par un orifice distinct (Gadoüdes, Lophius). Chez le Silure, le court faisceau (Pl. 2, fig. 4, f) fourni au tronc hyoïdeo-mandibulaire par la troisième portion du plexus, correspond probablement à ce rameau communicant; ici, il est simplement beaucoup plus court et se réunit au tronc dans l'inté-

' Stannius a donné le nom de trone hyoüdeo-mandibulaire à la continuation du « facial » après qu'il a donné les rameaux operculaire et adducteur palatin, ei reçu le rameau communicant du trijumeau. Gorovowitsch (100) a appliqué ce nom à la branche tout entière. C'est dans le dernier sens qu'il est employé ici.

Rev. Suisse de Zool., T. 6. 1898. 
rieur du crâne. La même explication doit peut-être s'appliquer aux cas où les branches du complexe ne se séparent qu'après avoir traversé la paroi du crâne par un orifice commun.

Le tronc hyoïdeo-mandibulaire, ainsi constitué, se sépare de la région moyenne et ventro-latérale du plexus ganglionnaire en se dirigeant en avant et un peu vers l'extérieur. Il s'engage dans un long canal oblique qui traverse l'os pétreux et débouche contre la face médiale (interne) des muscles élévateurs branchiaux. Tout en descendant légèrement, il s'avance entre la paroi cranienne et ces muscles, puis contourne le bord antérieur de ces derniers. Il prend alors une direction presque perpendiculaire à l'axe principal du corps et s'étend à la face inférieure de l'os hyomandibulaire $(\mathrm{Hm})$ en longeant le bord postérieur de l'adducteur palatin. Enfin, il traverse l'os hyomandibulaire (fig. 6) et vient émerger à sa face externe, par un orifice situé un peu en arrière de la crête $(\mathrm{CHm})$ que forme cet os pour l'origine de l'adducteur mandibulaire profond.

Avant ce point, le tronc hyoïdeo-mandibulaire a émis les deux rameaux suivants :

$1^{\circ}$ Rameau des muscles adducteur palatin et extenseurs du barbillon (Pl. 2, fig. 3, 4, rap). Il se détache du tronc un peu après que ce dernier a quitté le canal de l'os pétreux. Il est très facilement visible, tout au moins à son origine, où il est relativement fort. On peut déjà l'apercevoir par transparence, sous l'aponévrose qui recouvre dorsalement l'adducteur palatin. Il chemine, en effet, vers l'avant, plutôt médialement, entre ce muscle et son aponévrose, parallèlement au bord supérieur de l'os hyomandibulaire. Il diminue rapidement de volume, car il abandonne plusieurs filets qui s'enfoncent dans la masse musculaire.

Parmi les ramifications livrées au muscle adducteur palatin, la plus importante sort du rameau très près de son origine et se divise beaucoup; les autres sortent plus distalement, l'une 
d'elles atteint généralement aussi une grande longueur et montre une fausse anastomose avec le nerf sphéno-palatin.

Le rameau se prolonge à la surface de l'adducteur palatin en un filet de plus en plus mince, qui, se rapprochant davantage de la ligne médiane, traverse l'aponévrose et atteint le bord latéral du muscle extenseur profond du barbillon. (Pl. 2, fig. 5, 6, reib). A cet endroit, il s'en détache une ramification grêle (resb), allant obliquement en avant et en haut pour entrer dans le muscle extenseur supérieur à sa face dorsale. Ces deux nerfs terminaux se ramifient chacun dans l'un des muscles.

Stannius dit que le rameau destiné au muscle adducteur palatin a été observé chez huit genres de Poissons osseux ${ }^{1}$, mais il ne parle pas du Silure. En revanche, il attribue l'innervation du muscle qui tire le barbillon vers l'extérieur, à un rameau fourni par le tronc maxillaire commun ${ }^{2}$. Cette indication me paraît erronée; il n'est pas douteux que les deux muscles extenseurs soient actionnés par un rameau du tronc hyoïdeo-mandibulaire, qui innerve auparavant le muscle adducteur palatin.

$2^{\circ}$ Rameau des muscles adducteur hyomandibulaire, élévateur et adducteur de l'opercule (fig. 3, 4, 5, rof). Ce rameau important sort aussi du tronc hyoïdeo-mandibulaire lorsque celui-ci commence à descendre contre la face interne de l'os hyomandibulaire, mais il est émis par le bord postérieur du tronc et à quelque distance de la naissance du premier rameau. Il se dirige directement en arrière, en passant à la face interne de l'hyomandibulaire, entre cet os et la membrane qui va du muscle adducteur palatin, en avant, à l'adducteur hyomandibulaire, en arrière. Il s'étend ensuite à la face externe des adducteurs hyomandibulaire et operculaire situés l'un derrière l'autre, et à la face interne de l'élévateur operculaire, qui recouvre ces deux

P. 61 : Lucioperca, Cottus, Trigla, Scomber, Caranx, Belone. Esox, Cyprinus.

${ }^{2}$ P. 41. 
muscles. Il émet tout d'abord un filet pour le premier de ces muscles, et quelquefois un second un peu plus en arrière. Enfin, après s'être accolé momentanément au rameau operculaire du vague (rov), il se partage en trois ou quatre filets principaux allant dans l'élévateur et l'adducteur de l'opercule.

L' « anastomose " entre le rameau operculaire du tronc hyoïdeo-mandibulaire et celui du vague avait été trouvée par Stannius (16) chez Diodon, et par Büchner (9), puis StanNius chez les Cyprins. Vetter l'indique chez la Perche.

Remarquons, enfin, le fait que les deux muscles antagonistes de l'opercule sont pourvus par le même rameau. VETTER (48, p. 502) et GoRonowitsch ont trouvé un rameau destiné aux adducteurs hyomandibulaire et operculaire, et un ou plusieurs filets allant directement du tronc à l'élévateur operculaire. Chez Silurus, je n'ai pas pu découvrir de filet indépendant pour ce dernier muscle.

Parvenu à la face externe de l'os hyomandibulaire, le tronc hyoïdeo-mandibulaire se divise bientôt en deux fortes branches, l'une, dirigée en avant et en bas, se bifurque un peu plus loin, c'est le nerf mandibulaire (Pl. 2, fig. 3, 4, md), l'autre, dirigée en bas et en arrière et traversant l'os hyomandibulaire pour venir à sa face interne, constitue le nert hyoüdien (hy).

\section{Nerf mandibulaire.}

Le nerf mandibulaire, en descendant sur l'os hyomandibulaire, ne tarde pas à se diviser en deux branches très inégales en volume. La branche supérieure ou branche mandibulaire externe (fig. 3, 4, 5, 6,9, mde) est de beaucoup la plus forte. Elle va en avant et en bas, en reposant sur le bord inférieur de la portion profonde de l'adducteur mandibulaire et arrive sur la face latérale de l'articulaire, où elle se place sous la branche 
mandibulaire externe du nerf maxillaire inférieur. Les deux branches, accolées l'une contre l'autre, cheminent vers l'avant en étant recouvertes par le fascia de l'adducteur mandibulaire et échangent quelques filets anastomotiques. Enfin, distalement, elles se séparent; la supérieure se rapproche du bord labial, tandis que la branche du tronc hyoïdeo-mandibulaire s'en éloigne, mais reste également superficielle. Ses ramifications terminales s'avancent sous la peau jusqu'à la symphyse de la mandibule, quelques-unes d'entre elles s'unissent avec celles du rameau labial du nerf maxillaire inférieur.

La branche inférieure, beaucoup moins volumineuse, représente la branche mandibulaire interne (fig. 3, 4, 5, 9, mdi), l'unique prolongement de la branche mandibulaire des autres Téléostéens (d'après Stannius). Elle parvient contre la face interne de l'os carré ( $Q d)$ en passant dans un canal dont l'orifice externe se trouve à la limite entre cet os et le préopercule (fig. $1,2,6 ; \mathrm{n}^{0} 2$ ), descend contre le stylo-hyal, et traverse le ligament très fort qui relie la région angulaire de l'articulaire avec l'interopercule (Pl. 3, fig. 9, LIop). Pour traverser ce ligament, elle s'aplatit et se divise au moins en trois parties situées à quelque distance les unes derrière les autres; celles-ci viennent converger en un même point sous l'articulaire, et reconstituent la branche unique. Ces différentes parties sont très fines et difficiles à suivre; parfois, on ne voit que l'une ou l'autre d'entre elles, et il semble alors très étrange que le nerf diminue subitement d'épaisseur, sans émettre de ramifications un peu importantes, et augmente plus loin d'une manière très sensible.

Arrivé sur l'articulaire, le nerf s'infléchit médialement et en haut, et longe la face interne de la mâchoire inférieure, légèrement plus bas que le bord inférieur du cartilage de MEckeL. Avant de se bifurquer, il croise extérieurement la branche interne du maxillaire inférieur, laquelle sort, divisée en deux parties, de la rainure du dental (p. 76 et fig. 3,9 ). Ses deux . 
rameaux terminaux restent rapprochés et se ramifient principalement dans le tégument de la région médiane de la mandibule. J'ai déjà insisté (p. 78) sur le fait que les branches internes du tronc hyoïdeo-mandibulaire et du maxillaire inférieur se croisent simplement et qu'en ce point elles n'entrent nullement en communication. Plus distalement, cependant, les derniers filets des deux nerfs présentent plusieurs liaisons, mais les filets qui en résultent paraissent se rendre tous dans la peau. L'innervation du muscle intermandibulaire est donc dévolue entièrement au maxillaire inférieur.

Le nerf mandibulaire émet, avant de se diviser en branches interne et externe, deux rameaux assez forts (fig. 3) qui, passant entre le muscle adducteur mandibulaire et l'os hyomandibulaire, atteignent le bord du préopercule, se replient vers le haut et se répandent dans la peau de la région latérale de la tête, où leurs ramifications forment un réseau compliqué.

Une particularité que Stannius (16, p. 64) mentionne aussi pour l'Anguille, est la division du nerf mandibulaire en deux branches, analogues quant à leur trajet aux deux branches interne et externe du maxillaire inférieur. Ces deux branches ne semblent contenir que des éléments sensibles, puisqu'elles ne prennent pas part à l'innervation des muscles intermandibulaire et génio-hyoïdien. Leur domaine terminal est franchement limité à la mandibule; les deux ensemble forment donc un tout comparable à la branche principale unique des autres Téléostéens. La nécessité d'une innervation abondante de la face externe de la mandibule (p. 77) rend parfaitement compréhensible la formation d'une branche mandibulaire complètement superficielle (externe). Elle explique aussi pourquoi cette formation nouvelle acquiert un développement beaucoup plus considérable que la branche interne qui conserve assez bien la situation de la branche mandibulaire des autres Poissons osseux.

FRIANT $(49$, p. 62 à 71$)$ a trouvé que la branche antérieure du 
tronc hyoïdeo-mandibulaire donne généralement naissance à deux nerfs: le nerf mandibulaire et le nerf dentaire, dont l'un ou l'autre est le plus fort. Le nerf dentaire semble avoir reçu une désignation peu appropriée et n'être qu'un rameau destiné au plancher de la cavité buccale, peu constant chez les Téléostéens. En tout cas, ces nerfs mandibulaire et dentaire ne peuvent pas être envisagés comme étant ce que nous avons appelé les branches externe et interne; seul, le trajet du nerf mandibulaire de Friant peut être assimilé au trajet de la branche interne chez le Silure.

Nerf hyoüdien (Pl. 2, 3, fig. 3, 4, 9, hy).

C'est la branche postérieure du tronc hyoïdeo-mandibulaire. Elle va obliquement en arrière et en bas, sur l'os hyomandibulaire qu'elle traverse par un orifice situé au bord inférieur de cet os et limité latéralement par le préopercule (fig. 1, 2, $6 ; n^{\circ} 3$ ). Puis elle croise, à leur face interne, le préopercule et l'interopercule; elle repose alors sur la membrane qui tapisse intérienrement la cavité branchiale (fig. 9, y). Arrivée derrière l'extrémité supérieure de l'épihyal (fig. 1, 9, Ehy), elle s'infléchit obliquement en avant et en bas, en suivant à une petite distance le bord supérieur de cet os et parvient sur la membrane branchiostège. Là, elle chemine en croisant les rayons non loin de leur base et en abandonnant de nombreuses ramifications (fig. 9). Son prolongement, considérablement réduit de volume, prend une direction plus longitudinale, descend légèrement pour passer entre les sixième et septième rayons branchiostèges et pénétrer dans le muscle hyo-hyoïdien inférieur (fig. 9, H hi) dans la masse duquel ses dernières ramifications se répartissent.

Pendant ce parcours, le nerf hyoïdien fournit de nombreux rameaux qui sont : 
$1^{\circ}$ Un rameau sortant du bord postérieur du nerf, près de la place où celui-ci sort du tronc hyoïdeo-mandibulaire. Il se dirige en arrière sur le revêtement cutané de la face interne de l'opercule (fig. 3 et 4 ).

$2^{\circ}$ Un rameau (fig. 3) naissant plus loin et destiné soit à la partie du muscle hyo-hyoïdien fixée sur l'opercule, soit à la membrane qui recouvre du côté interne l'interopercule et le rayon branchiostège remplaçant le subopercule.

$3^{\circ}$ Plusieurs petits rameaux dirigés en avant et se distribuant dans la peau, au-dessous et autour de l'épihyal.

$4^{\circ}$ De nombreux rameaux destinés à la membrane branchiostège. Ces derniers, plus ou moins forts, sortent au niveau des espaces situés entre les rayons. Dans le reste de leur parcours, ils ne sont pas parallèles à ceux-ci, mais ils se dirigent obliquement en arrière et vers la ligne médiane. Ceux destinés à la partie antérieure de l'appareil branchiostège s'avancent médialement, sur un certain trajet, du côté de la face interne. Ils se divisent tantôt en deux, tantôt en plusieurs filets allant dans la musculature qui relie les rayons entre eux, ainsi qu'à l'opercule d'une part, et à l'os hyoïde d'autre part; enfin, ils innervent aussi le revêtement cutané tant interne qu'externe de la membrane.

Stannius $(16$, p. 62$)$ ne parle pas de l'innervation du muscle génio-hyoïdien par un rameau terminal du nerf hyoïdien. GoroNowiTsch (100) dit seulement que ce nerf émet, outre un rameau operculaire, des branches destinées à la musculature de la membrane branchiostège. FRIANT (49) et VETTER (48) admettent tous deux que le muscle génio-hyoïdien est partiellement innervé par une branche du nerf hyoïdien.

Le dernier auteur a pu se convaincre que, notamment chez Esox, le nerf hyoüdien innerve la partie supérieure, la plus grande, du muscle en question (p. 515). La partie inférieure du même muscle serait actionnée par une branche issue de la réu- 
nion d'un rameau du trijumeau et d'un rameau du «facial » ', laquelle livrerait aussi des filets au muscle intermandibulaire Admettant que les éléments du facial, ainsi réunis à des éléments du maxillaire inférieur, pouvaient bien n'être que des fibres sensibles, il supposa que la faible partie antérieure (inférieure) du muscle génio-hyoïdien pourrait dériver d'un autre segment musculaire que la portion postérieure innervée par le nerf hyoïdien ${ }^{2}$. Ruge (102, p. 283), trouve cette opinion peu satisfaisante, mais il ne la considère pas encore comme réfutée.

Le Silure présente, sous ce rapport, une disposition intéressante. L'innervation de son génio-hyoïdien dépend de la branche externe du maxillaire inférieur et non pas de parties réunies de la branche interne et du «facial» (v. p. 78). Donc ici, le génio-hyoïdien appartient franchement au domaine du maxillaire inférieur, comme l'intermandibulaire. Le nerf hyoïdien, après avoir passé de la face interne à la face externe de la membrane branchiostège, arrive très près de l'origine du génio-hyoïdien, mais cependant médialement par rapport à celle-ci. Les fibres musculaires dans lesquels il pénètre alors appartiennent au muscle hyo-hyoüdien, dont une partie de l'origine est peu distincte de celle du génio-hyoïdien.

L'hypothèse de VETTER ne peut donc pas être soutenue après l'examen de Silurus glanis. Il est probable que la prétendue région du génio-hyoüdien innervée, chez certains Poissons, par le nerf hyoïdien n'est qu'une partie du hyo-hyoïdien inférieur, dont !'origine est difficile à distinguer de celle du génio-hyoïdien, comme c'est le cas chez le Silure; ou bien il s'agit d'une

1 Vetter appelle facial le nerf que nous désignons comme branche hyoïdeomandibulaire du trijumeau-facial.

${ }^{2}$ Vetter p. 537 : a Dagegen spricht die Innervirung der untern (vordern) Hälfte des leiztern (Geniohyoideus) durch den Maxillaris inf. dafür, diesen Theil noch als Differencirung von Csv ${ }_{1}$, mitbin als zum Intermandibularis gehörig zu betrachten. Doch lässt sich in Ermangelung von erläuternden Zwischenformen diese Frage nicht bestimmt beantworten. : 
partie de ce premier muscle qui se serait adjointe secondairement au deuxième, phénomène qui n'a pas eu lieu chez le Silure.

\section{Branches ascendantes du trijumeau-facial.}

Du plexus du trijumeau-facial, naissent une forte branche et plusieurs rameaux beaucoup moins importants, que l'analogie de leur naissance et leur trajet dorsal permettent de ranger sous le titre indiqué. La forte branche nait le plus postérieurement et constitue la branche dorsale du trijumeau-facial ou nerf de Weber; les rameaux qui sortent plus en avant peuvent être réunis sous le nom de rameaux ascendants antérieurs.

\section{Rameaux ascendants antérieurs (fig. 3, rasc).}

Ces rameaux partent de la région proximale du ganglion de GASSER, généralement sous forme de quatre cordons grêles, situés les uns derrière les autres et parfois très rapprochés.

Leur origine dépend des différentes parties du plexus (v. p. 55), en tout cas de trois d'entre elles. Le filet antérieur, très faible, sort du bord dorsal du ganglion, soit de la partie qui forme principalement les branches ophtalmiques. Les deux filets suivants viennent de la face médiale de la masse ganglionnnaire, soit des deuxième et troisième portions, lesquelles alimentent surtout les branches maxillaires. Enfin, le quatrième filet, plus fort que les précédents, tire son origine, en partie de la deuxième portion, en partie du nerf de WEBER contre le bord antérieur duquel il est appliqué dans la première partie de son trajet. De cette manière, il se peut que le quatrième filet reçoive des éléments de la première portion, puisque c'est surtout de celle-ci que le nerf dorsal provient.

Ces quatre filets se rapprochent pour former deux rameaux; tantôt ils se fusionnent deux à deux, tantôt ce sont les trois 
antérieurs qui se réunissent, tandis que le postérieur constitue un rameau à lui seul. Les deux rameaux se dirigent en haut et en avant, contre la paroi du crâne, en émettant plusieurs filets qui se prolongent antérieurement et se perdent dans le tissu graisseux de la cavité cranienne. Ils pénètrent ensemble dans la partie supérieure de l'os pétreux, parviennent dans le squameux, à l'intérieur duquel ils divergent, puis viennent se rejoindre et se fusionner complètement à la face externe de cet os. Le rameau qu'ils constituent s'infléchit en arrière, pour descendre entre la peau et la partie postérieure de l'adducteur mandibulaire et se-perdre dans la peau qui recouvre le préopercule et l'opercule. En passant sur la face supérieure du muscle adducteur mandibulaire, il envoie quatre ou cinq filets qui descendent dans le revêtement cutané de la partie latérale de la tête.

Les rameaux ascendants peuvent présenter des modifications nombreuses, aussi les cas d'asymétrie sont-ils très fréquents. Les dispositions décrites ici m'ont paru être les plus constantes. Il est certain que ces rameaux ne contiennent que des éléments sensibles et que ceux-ci sont fournis par les divers composants du plexus.

Nerf de Weber (fig. 3, 4, nWb).

Ce nerf a été découvert par E.-H. Weber (1820), chez Silurus glanis. Il a été trouvé par Desmoulins chez les Gadoïdes et les Siluroïdes. Stannius $(16$, p. 49$)$ l'a rencontré chez environ vingt-cinq espèces. Il le décrit sous le nom de rameau latéral du trijumeau et dit qu'il sort du plexus des racines du trijumeau et du facial par deux branches distinctes. Cet auteur ne précise pas davantage ses indications sur la provenance des deux composants. Il mentionne le fait que, particulièrement chez le Silure, leurs éléments constitutifs sont exclusivement 
des « tubes primitifs fins » et que l'excitation galvanique ne provoque aucune contraction musculaire. Chez la plupart des Poissons, le nerf reçoit, encore à l'intérieur de la cavité cranienne, un filet venant des racines du vague; Silurus glanis ne montre aucune trace de ce rameau communicant (WEBER et Cuvier nient également son existence).

Friant $(49$, p. 98) dit que, généralement, son «nerf facial» (probablement une branche de l'ophtalmique) « fournit un rameau qui s'élève dans la cavité cranienne et en ressort pour se répandre dans les canaux muqueux de la tête ou dans la peau et les muscles du tronc. Ce rameau fait défaut chez la Lotte, et il est remplacé par un tronc qui naît du ganglion du Triju-

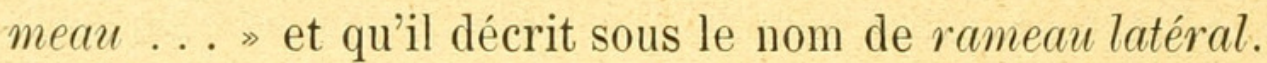

H.-B. Pollard (86, p. 529), en traitant de l'innervation du système de la ligne latérale, attribue le nerf de WEBER entièrement au facial : "From a posterior dorsally placed ganglionic extension arises the great Ramus dorsalis recurrens facialis (often wrongly called trigemini). » Ses recherches, qui n'ont porté généralement que sur de jeunes spécimens, ont montré l'existence d'un rameau communicant entre la première ramification du rameau latéral du vague et le nerf récurrent du facial, chez Clarias, Auchenaspis biscutatus, Callichthys paleatus, Chaetostomus guairensis. Cette ramification, nommée rameau supra-temporal, manque à Trichomycterus tenuis.

D'après GoRonowitsch $(100$, p. 27) le nerf de WEBER, chez la Lotte, sort du ganglion du facial, mais il reçoit deux branches connectives formées, l'une d'éléments du trijumeau postérieur et de la racine ventrale du facial, l'autre, d'éléments du trijumeau antérieur. "Le nerf de WEBER est donc constitué par des fibres de tous les nerfs segmentaux du complexe. ”

Les indications de la littérature nous laissent, par conséquent, dans l'embarras, soit par leur insuffisance, soit par leurs divergences et leur manque de coordination. 
Chez le Silure, le nerf de WEBER doit certainement être regardé comme une branche du trijumeau-facial. Il s'élève contre la face médiale et contre la partie postérieure du plexus ganglionnaire, et là, on peut reconnaître qu'il est formé de la réunion de deux composants, très inégaux en volume.

Le composant postérieur est de beaucoup le plus fort. A la sortie de la moelle allongée, il forme un ruban aplati, enchàssé entre les deux premières portions du complexe qui sont situées au-dessous et la troisième portion située au-dessus (v. p. 55). Un peu en arrière de la sortie, on peut voir que ce composant est alimenté en grande partie par la première portion, mais reçoit aussi un contingent d'éléments assez considérable de la deuxième. La première portion renferme la majorité des éléments du facial et constitue en grande partie le tronc hyoïdeo-mandibulaire, tandis que la deuxième portion doit contenir des éléments du trijumeau antérieur et s'en va principalement dans le tronc maxillaire commun (p. 67). Le composant vient bientôt émerger entre la deuxième et la troisième portion à la face médiale du plexus, tout près de la moelle allongée. Il se dirige en haut et un peu en avant et reçoit le composant antérieur, beaucoup moins volumineux.

Ce dernier représente un faible ruban, formé de deux ou trois filets accolés, situés les uns derrière les autres et sortant de la face médiale de la troisième portion. Cette portion doit contenir des éléments du trijumeau postérieur et du facial; ceux qu'elle fournit au nerf de WEBER ne dépendent très probablement que du trijumeau postérieur. Le composant antérieur s'élève en s'infléchissant un peu en arrière et se joint au fort composant postérieur à une petite distance du bord supérieur du plexus.

Comme pour l'origine des autres branches du complexe, on doit admettre la possibilité d'échanges d'éléments à l'intérieur du cerveau. Je n'indique la répartition probable des trijumeaux antérieur et postérieur que d'après la position des 
portions à leur sortie de la moelle allongée et d'après les relations qu'elles montrent ensuite les unes avec les autres. Ce qui est certain, c'est que le nerf de WEBER ne reçoit pas ses fibres d'une seule région du plexus, mais au contraire de trois régions différentes, et que ces dernières appartiennent au facial et au trijumeau. De plus, il est très vraisemblable que le nerf de WEBER, chez le Silure, tire son origine des trois nerfs segmentaux du complexe. Comme qu'il en soit, c'est le facial qui lui fournit le plus fort contingent; le trijumeau antérieur vient enensuite; le trijumeau postérieur n'envoie probablement que le faible composant antérieur.

De même que Stannius, je n'ai pas trouvé de rameau communicant entre le nerf latéral du vagne et le nerf de WEBER. On sait, d'après PoLlard, que ce rameau manque aussi chez Trichomycterus tenuis.

Le nerf de WEBER s'élève au-dessus du plexus, sous l'aspect d'un tronc très volumineux. D'après Stannius (p. 50), il formerait, en devenant indépendant, un ganglion assez distinct. Mes recherches ne me permettent pas de confirmer cette indication; le nerf, d'abord aplati, devient cylindrique, mais ne présente pas de renflement appréciable en cet enđroit. Appliqué contre la paroi latérale de la cavité cranienne, il se dirige en haut, puis un peu en arrière et médialement, et traverse le plafond du crâne par un canal de l'occipital supérieur '. L'orifice externe de ce dernier est une fente longitudinale, étroite (Pl. I, fig. 1, nWb), située à la base de la crête sagittale de l'occiput. En arrière de l'orifice, la crête occipitale présente une dépression bien visible, ovale, ayant son plus grand diamètre dans le sens antéro-postérieur. Là, le nerf dorsal reçoit les rameaux dorsaux des nerfs spinaux II et III, et montre un élargissement consi-

1 Polland (86) a trouvé, chez les espèces qu'il a étudiées, que le canal de sorlie était dans le pariétal. 
dérable ayant un peu l'aspect d'un ganglion (Pl. 2, fig. 3). Cependant, le volume de la branche ne paraissant pas s'accroître, il semblerait qu'il y ait plutôt aplatissement du nerf dans le sens transversal. Le renflement commence quelquefois déjà à l'intérieur de l'occipital supérieur, donc avant l'arrivée du rameau venant du deuxième nerf spinal, d'autres fois, il com mence plus en arrière, vers l'arrivée du rameau dorsal du quatrième nerf spinal. Il n'est jamais large, mais il est très étendu ; dans le sens dorso-ventral, il offre ses dimensions maximales aux points où il reçoit les éléments venant des nerfs spinaux. La figure 3 représente un cas où la plus forte dimension est atteinte au point d'arrivée d'un rameau formé par des parties des deux premiers nerfs spinaux qu'il reçoit (IId et IIId), tandis que le renflement est insignifiant à la place où aboutissent des filets plus forts venant des rameaux dorsaux des nerfs spinaux III et IV.

Les rameaux dorsaux des nerfs spinaux II et III sortent respectivement des deuxième et troisième ganglions spinaux lesquels sont formés par la racine dorsale de chacun des nerfs. Ils s'élèvent le long de la crête occipitale en se dirigeant un peu en avant. Avant d'atteindre le nerf de WEBER, ils se divisent généralement, mais, sous ce rapport, on n'observe pas plus de constance d'un individu à un autre que de symétrie chez un même exemplaire. Tantôt, la plus forte partie du rameau postérieur vient se fusionner au premier rameau, immédiatement avant que celui-ci se joigne au nerf de WEBER, tantôt, ce sont les deux rameaux qui se bifurquent (fig. 3) et alors les deux ramifications extrêmes se rendent indépendamment dans le nerf de WeBER tandis que les deux ramifications intermédiaires se réunissent avant d'y pénétrer, tantôt enfin il y a formation de ramifications plus nombreuses s'anastomosant d'une manière variable. Souvent, le rameau postérieur est plus volumineux que le premier, mais ce fait n'est pas général. La seule chose 
constante est que tous deux pénètrent entièrement dans le nerf de WEBER.

De la partie renflée du nerf partent plusieurs filets allant en haut et en avant et qui peuvent être suivis dans les régions occipitale, pariétale et frontale où ils se perdent dans la peau.

Ensuite, la branche dorsale du trijumeau-facial se prolonge en arrière, en passant entre le muscle latéral du tronc et la membrane fibreuse qui, partant de la crête occipitale, relie entre elles les apophyses épineuses des vertèbres; elle n'est alors séparée de sa congénère de l'autre côté que par cette dernière membrane. Contrairement à l'opinion de Stannius (p. 51), le nerf de WEBER ne se trouve pas directement sous la peau, mais à une distance qui diminue d'avant en arrière. Il passe au niveau des extrémités supérieures des apophyses épineuses, dans l'angle formé par le fascia sagittal du muscle latéral et le fascia latéral du muscle grêle supérieur ' (schéma, p. 133, x). Ce dernier fascia est presque vertical en avant et devient peu à peu horizontal en arrière.

Durant son long trajet, le nerf de WEBER, est le collecteur des rameaux dorsaux de tous les nerfs spinaux à l'exception du premier. Celui-ci est très faible et s'élève sur le supra-occipital un peu en avant de l'orifice de sortie du nerf de WEBER. Quelquefois, pourtant, des filets du premier rameau dorsal s'unissent aux rameaux dorsaux suivants et pénètrent avec ceux-ci dans le nerf de WeBER. Les deux premiers rameaux dorsaux qui se jettent dans le nerf (Pl. 2, fig. 5, IId, IIId) sont inclinés en avant; le troisième monte presque verticalement ou se dirige

${ }^{1}$ Cunier et Valenciennes (p. 291 et 992 ) ont appelé ainsi la bande musculaire paire intercalee du côté dorsal entre les deux muscles latéraux. Chez Silurıs glanis, ¡’ai trouvé que cette bande comprend deux parties. L'une, en forme de lame verticale, est mince et haute, et s'étend de la crêtes occipitaie à la faible nageoire dorsale; l'autre, moins haute et plus large, va de la nageoire dor'sale a la nageoire caudale. 
légèrement en arrière; les suivants sont de plus en plus incliné en arrière. En général, chacun de ces rameaux se partage, non loin du ganglion spinal, en une partie antérieure et une partie postérieure plus faible. La partie postérieure entre quelquefois directement dans le nerf de WEBER; le plus souvent, elle s'unit à la partie antérieure du rameau dorsal suivant, là où celle-ci atteint le nerf de WEBER ou au-dessous. Très fréquemment, les rameaux dorsaux, au moment d'arriver dans la branche collectrice, forment un système de fines ramifications, plus ou moins compliqué. Dans tous les cas, les ramifications passent entièrement dans le nerf de WEBER et il ne se forme pas de nœuds aux points de jonction.

De son bord supérieur, le nerf de WEBER livre des rameaux (Pl. 2, fig. 5), qui, dirigés en arrière et en haut, passent entre les deux moitiés du muscle grêle supérieur ou entre le muscle grêle et le grand muscle latéral (schéma, p. 133, rnWb), pour venir innerver la peau de la région dorsale. Ces rameaux ne sont ordinairement pas dans le prolongement des rameaux dorsaux des nerfs spinaux. Leurs terminaisons sont souvent enchevêtrées.

Dans la région voisine de la nageoire dorsale, le nerf émet deux rameaux un peu plus forts que les autres; ils pourvoient aussi bien les muscles de cette nageoire que son revêtement cutané.

En arrière, le nerf de WEBER diminue graduellement de volume; il va jusqu'à la nageoire caudale, où son filet terminal et ses dernières ramifications forment un réseau simple.

L'origine du nerf de WeBER et son renforcement par les rameaux dorsaux des nerfs spinaux justifient parfaitement l'opinion avancée par STANnius (p. 72), que les « Schedelhöhlenäste " et le «Ramus lateralis » des Poissons osseux correspondent aux rameaux dorsaux des nerfs spinaux. Chez le Silure, on peut voir, d'après la description détaillée donnée plus haut, 
que le nerf de WEBER doit contenir des éléments des rameaux dorsaux de tous les nerfs segmentaux prenant part au complexe trijumeau-facial. Quant aux rameaux ascendants antérieurs, il faut probablement les considérer, en partie comme des ramifications secondaires des rameaux dorsaux du trijumeau-facial, en partie comme des ramifications intra-craniennes des branches du complexe.

\section{Nerf acoustique.}

(Pl. 2, fig. 3, 4, ac.)

Le nerf acoustique sort de la moelle allongée, à son bord latéral et inférieur, en arrière du trijumeau-facial et en avant du glosso-pharyngien. C'est un large ruban aplati, divisé aussitôt en une partie antérieure et une partie postérieure.

La partie antérieure (fig. $4 \mathrm{ac}_{1}$ ) émerge immédiatement derrière la racine ventrale du facial, mais elle en est complètement indépendante. Elle constitue un ruban aplati, dirigé latéralement et un peu en avant sur le plancher de la cavité cranienne et donne naissance à deux cordons qui se bifurquent à leur tour. Le premier de ces cordons, le plus faible, donne un rameau antérieur se repliant un peu en haut pour entrer dans l'ampoule du canal semi-circulaire antérieur et un rameau postérieur destiné à la région antérieure de l'utricule. Le deuxième cordon, plus large, envoie son faible rameau antérieur également dans la région antérieure de l'utricule et son rameau postérieur dans l'ampoule du canal externe.

La partie postérieure $\left(\mathrm{ac}_{2}\right)$ du nerf acoustique sort de la moelle allongée en avant du composant antérieur du nerf glossopharyngien; elle est beaucoup moins considérable que la partie antérieure, mais son trajet est plus long. Elle va d'abord en arrière, le long du bord inférieur de la moelle allongée, puis s'infléchit latéralement et se place au-dessus du composant anté- 
rieur du glosso-pharyngien (fig. 4). Elle se divise en deux rameaux recourbés en avant; l'antérieur va dans le saccule, l'autre dans l'ampoule du canal semi-circulaire postérieur.

En résumé, la partie antérieure de l'acoustique se distribue à l'ampoule antérieure, à l'ampoule externe et à l'utricule, tandis que la partie postérieure innerve le saccule et l'ampoule postérieure.

Je n'ai jamais trouvé de communication entre l'acoustique et le trijumeau, comme Weber (2) en a indiqué une pour Silurus glanis. A leur sortie du cerveau, le trijumeau-facial et l'acoustique sont très rapprochés, mais il n'y a pas de faisceau distinct passant d'un nerf à l'autre.

En outre, ce qui vient d'être décrit comme partie postérieure de l'acoustique correspond, sans doute, à l'auditif' accessoire de Weber. La distribution répond bien à celle indiquée par cet auteur; quant à l'origine, elle n'est pas différente de celle de la partie antérieure, les deux cordons nerveux sont en contact à la sortie de la moelle allongée, le postérieur se dirige en arrière jusque vers le glosso-pharyngien, mais il n'a aucune relation ni avec ce dernier, ni avec le vague.

\section{Nerfs gLosso-Pharyngien ET VAGUE}

Les nerfs glosso-pharyngien et vague du Silure sont parfaitement indépendants l'un de l'autre dès leur sortie du cerveau. Cependant, il semble préférable de ne pas les traiter séparément, soit parce qu'ils se partagent l'innervation d'un même domaine : les arcs branchiaux et leur musculature, soit parce que nombre de travaux antérieurs établissent, par d'autres voies, leur communauté d'origine.

Desmoulins (3) prétendait, en 1822, que le glosso-pharyngien manquait aux Poissons, car il regardait comme branche 
antérieure du vague, le nerf définitivement reconnu plus tard comme l'homologue du glosso-pharyngien.

De même, LoNgET (12, t. II, p. 235) admettait, en 1842, que " dans les Poissons il n'existe point de nerf glosso-pharyngien proprement dit».

Néanmoins, la plupart des auteurs reconnaissent l'existence du glosso-pharyngien chez les Poissons, ainsi que sa parenté plus ou moins étroite avec le vague.

Büchner (9) dit, en 1835, que chez le Barbeau, le glossopharyngien naît immédiatement devant le lobe du vague et qu'il est presque contigu à la racine supérieure de ce nerf.

D'après Stannius (16, p. 74), le glosso-pharyngien est indépendant du vague chez la plupart des Poissons osseux, mais, chez quelques-uns d'entre eux, il y a encore entre leurs racines des rapports très étroits.

Pour Gegenbaur (31, p. 704) : "Le premier rameau branchial du nerf vague prend, peu à peu, chez les Sélaciens, les Ganoïdes et Téléostéens la signification d'un nerf indépendant... Il constitue le nerf glosso-pharyngien. »

D'après BeaUREGaRD (56), le glosso-pharyngien, chez le Ceratodus Forsteri, n'est encore que la branche antérieure du vague.

D'après GoRonowitsch (81), les deux nerfs naissent séparément chez Acipenser ruthenus.

Enfin, rappelons encore une indication récente et très précise de B. Haller (101, p. $\overline{9} 5$ et 96$)$ : «Der Glossopharyngeus ist ein stets vor dem Vagus und in gleicher Höhe mit diesem aus der Oblongata abgehender Nerv, der auch durch seinen äusseren Abgang als ein der Vagusgruppe angehörender Nerv sich zu erkennen giebt. Auch was seinen Ursprung anbelangt, so ist er als ein Abkömmling der Vagusgruppe zu betrachten. » Cet auteur ajoute que le lobe sensible du glosso-pharyngien est détaché secondairement, aussi bien chez les Cyprins que chez la Lotte, du lobe sensible du vague. 
Cet aperçu historique, très abrégé, donne cependant une idée des diverses opinions qui ont été émises sur la question et montre que toutes ces opinions tendent à prouver la co-dépendance du glosso-pharyngien et du vague.

Ces deux nerfs présentent un état beaucoup moins compliqué que le trijumeau-facial, aussi les indications des auteurs présentent-elles moins de divergences que lorsqu'il s'agit de ce dernier complexe.

\section{NerF GLOSSO-PHaRYNGIEN}

(Pl. 2, 3, fig. 3. 4, 7, gp).

Le glosso-pharyngien sort de la partie latérale et postérieure du bulbe rachidien par deux composants distincts de ceux du vague.

Le composant antérieur est faible et sort juste au-dessus de la partie postérieure du nerf acoustique; il se dirige en arrière en suivant le bord inférieur et externe du bulbe et se joint au composant postérieur. Celui-ci quitte la moelle allongée audessous de la portion antérieure du vague, avec laquelle il est d'abord si étroitement accolé qu'à première vue il semble en dépendre. En le suivant attentivement, on reconnaît qu'il longe inférieurement la portion radicale du vague et qu'il émerge du cerveau au-dessous de celle-ci, soit plutôt de la face ventrale que de la face latérale. Au point où les deux composants du glosso-pharyngien s'unissent, un faisceau peu volumineux et très court, provenant du composant antérieur du vague, s'adjoint à eux (fig. 3 et 4 ).

Le nerf, ainsi constitué, n'a qu'un court trajet dans la cavité du crâne. Il chemine sur le plancher de cette dernière, à peu près perpendiculairement à la direction longitudinale. Après avoir passé sous l'ampoule du canal semi-circulaire postérieur, il s'engage dans un canal particulier, qui traverse l'occipital la- 
téral en obliquant fortement vers l'avant. L'orifice externe du canal se trouve à environ $0,75 \mathrm{~cm}$. en avant de celui du vague.

Immédiatement à sa sortie du conduit osseux, le nerf, continuant à aller en avant, forme un ganglion ( $g g p$ ) aplati et appliqué contre la face externe de l'occipital latéral.

On doit remarquer qu'avant le renflement, le nerf se partage en deux parties, dont la supérieure seule, un peu plus forte que l'autre, prend part à la formation ganglionnaire. La partie inférieure forme une sorte d'anse au-dessous du ganglion, sans cependant s'éloigner de son bord inférieur et rejoint la première immédiatement après le renflement (fig. 3). Ce dernier est en communication avec le cordon sympathique situé au-dessous par des filets très minces, au moins au nombre de deux.

Le glosso-pharyngien est sensiblement plus volumineux après le ganglion qu'avant. Il continue sa marche en avant, en passant entre la face externe de l'os pétreux et le muscle rétracteur branchial antérieur (fig. 7, $R b a$ ), puis, s'infléchissant latéralement, s'introduit entre les muscles élévateurs des deux arcs branchiaux antérieurs.

Jusque-là, le nerf fournit quelques petits filets musculaires. Le premier part immédiatement à la sortie du crâne et entre probablement dans le rétracteur branchial antérieur; mais je n'ai pu m'assurer de ce fait, le nerf ayant été cassé. Le suivant sort de l'anse formée par la portion du nerf qui ne prend pas part à la formation du ganglion; il se dirige latéralement et vient se perdre dans le même muscle, ainsi que un ou deux filets qui naissent un peu plus en avant. Un filet moteur est encore livré par le nerf au moment où il s'engage entre les deux élévateurs branchiaux; il s'élève un peu et pénètre dans l'élévateur du premier arc. Enfin, un autre rameau est émis un peu avant le dernier, mais il n'est pas moteur; il contourne par devant l'insertion de l'élévateur du premier arc, se replie en arrière, envoie un filet mince à la peau qui tapisse la cavité branchiale 
et pénètre dans le premier arc branchial. Il s'étend à la face antérieure de celui-ci, dans la muqueuse qui le recouvre, puis passe par-dessus le nerf qui lui a donné naissance et se perd sur le bord supérieur de l'arc (fig. 3 et 4 ).

Le glosso-pharyngien du Silure ne fournit pas de branche antérieure allant dans la muqueuse du palais et suivant l'arc hyoüdien, comme c'est le cas chez la plupart des Poissons osseux. Stannius $(16$, p. 76 et 77$)$ a également remarqué que cette branche hyoïdienne manque chez le Silure et le Brochet, et l'a trouvée dans un état très rudimentaire chez Belone. Pour le moment, il semble impossible de pouvoir avancer une explication ou une conjecture quelconque sur ces curieuses exceptions à la disposition métamérique, rien d'anormal dans l'arc hyö̈dien, ni dans le premier arc branchial ne paraissant les motiver.

Après avoir passé entre les deux élévateurs branchiaux, le glosso-pharyngien s'infléchit latéralement et en arrière et entre dans la rigole creusée le long du bord périphérique du premier arc branchial. Il est alors situé au-dessus de la veine branchiale. Avant le coude de l'arc, il sort de la rainure et fournit un filet descendant le long de la courbure interne. Ce filet va dans la muqueuse qui recouvre la face antérieure de l'arc et dans celle qui revêt les saillies dentiformes dont le premier arceau, de même que les suivants, est pourvu du côté de la cavité buccale.

Le nerf descend ensuite sur la face antérieure de l'arc, non loin du bord convexe, et vient se loger dans la rigole du long cérato-branchial. Là, il se divise en trois filets principaux qui courent parallèlement les uns aux autres, envoient des filets à la muqueuse de l'arc, à la double rangée de lamelles branchiales, aux vaisseaux sanguins qu'ils accompagnent, et s'atténuent de plus en plus à mesure qu'ils se rapprochent de l'hypobranchial. Deux de ces filets situés près des lamelles branchiales, semblent pourvoir plus particulièrement à leur innervation; ils se terminent a vec le cérato-branchial. L'autre filet (fig. 2, gp) plus fort, 
fournit très peu de rameaux durant le même trajet, aussi est-il encore assez volumineux lorsqu'il pénètre dans le muscle pharyngo-arcuo-hyoïdien. Il se prolonge à la face inférieure de l'hypobranchial et s'élève devant le bord antérieur de cet os pour parvenir dans la muqueuse qui revêt la face supérieure de la copule antérieure et de l'hypohyal.

\section{Nerf vague.}

La littérature concernant le nerf vague des Poissons est excessivement volumineuse. La distribution nettement métamérique d'une partie de ce nerf, après avoir été mise en relief par Gegenbaur dans sa théorie de la polymérie du vague, a suscité un grand nombre de recherches sur les centres d'origine. Mais, si la répartition du vague dans les arcs branchiaux et dans les viscères, ainsi que la question de son origine, ont été très travaillées, il n'en a pas été de même de l'innervation des muscles branchiaux. Enfin, il existe de fréquentes contradictions dans les indications des auteurs au sujet des rapports du vague avec les autres nerfs.

Sortie du cerveau. - Le nerf vague sort du bord latéral et supérieur de la moelle allongée par deux composants situés l'un derrière l'autre (fig. 3 et 4 ).

Le composant antérieur (fig. 4 cav) est moins considérable que le composant postérieur (cpv), il est compact et sort du bord inférieur et postérieur du lobe de la moelle allongée, audessus du composant postérieur du glosso-pharyngien.

Le composant postérieur, un peu plus volumineux, quitte la moelle allongée légèrement plus haut, c'est-à-dire plus près de la face dorsale, mais passablement en arrière (au moins $3 \mathrm{~mm}$.). Il sort d'une place présentant un renflement à peine sensible, correspondant peut-être au lobe du vague que BAUDELOT (38) a trouvé chez quelques Poissons osseux. Ce point de sortie se 
trouve dans le même plan vertical que le bord postérieur de l'ouverture du quatrième ventricule. A sa sortie du cerveau, le composant postérieur n'est pas constitué d'une seule masse d'éléments, mais d'un fort faisceau supérieur et de deux faisceaux inférieurs. Ces derniers sont très grêles, ils naissent un peu au-dessous et en avant du faisceau supérieur auquel ils s'unissent bientôt.

E.-H. Weber (2) a vu le vague du Silure naître par trois racines. Stannius (16) parle de deux portions de racines : une antérieure comprenant un seul faisceau et une postérieure formée de deux cordons épais qui correspondent évidemment aux deux dernières racines de WEBER. Le même auteur montre que, chez les Poissons osseux, la racine antérieure contient exclusivement des éléments sensibles, mais il ajoute (p.82) : "Zweifelhaft blieb mir das Verhalten der Primitivröhren nur bei Silurus ", car il sembie que, chez ce Poisson, des éléments fins soient mêlés aux éléments larges, à double contour ; la portion postérieure renferme des éléments des deux sortes, les éléments moteurs y dominent.

Nous devons admettre que des différences individuelles assez fréquentes peuvent se rencontrer dans les parties centrales du vague. Le composant antérieur présente le moins de variations, tandis que le composant postérieur est formé de deux ou trois groupes d'éléments qui tendent à se fusionner en un seul. Ainsi, dans le dessin de WEBER (Pl. V, fig. 30), les deux racines postérieures sont situées l'une derrière l'autre; je ne les ai jamais vues dans cette situation. Généralement, elles sont superposées et se fusionnent avant de s'unir au composant antérieur, ce qui justifie parfaitement l'idée de Stannius de les regarder comme parties d'un même composant. C'est donc par l'examen d'un cas exceptionnel que WEBER a été conduit à dire que le vague naît par trois racines. Enfin, le composant postérieur peut être plus compliqué, son cordon inférieur (ou postérieur) pouvant être 
représenté par deux faisceaux grêles, très rapprochés, ce que je n'ai constaté exactement qu'une seule fois.

Les observations précédentes et l'indication de Stannius (confirmée d'ailleurs par la répartition périphérique), que la portion postérieure du vague est formée aussi bien d'éléments sensibles que d'éléments moteurs, montrent bien que les parties dont la réunion constitue le vague ne sont pas de véritables racines, comme celles que l'on distingue généralement dans les nerfs spinaux, et qu'il est préférable de les désigner sous le nom de composants. Le composant antérieur, formé exclusivement de fibres sensibles, ne peut être considéré que comme une partie de la racine dorsale du vague, racine qui, d'après GEGENBAUR, est elle-même le produit de la concrescence d'un certain nombre de racines dorsales homologues à des racines dorsales de nerfs spinaux. Le composant postérieur comprend la racine ventrale du vague, c'est-à-dire l'ensemble des racines motrices des nerfs segmentaux qui ont formé le vague, plus une partie de la racine dorsale de ce nerf. Cette manière de voir est d'ailleurs confirmée par la présence d'un rameau dorsal partant du composant postérieur et dont nous allons parler.

Trajet intra-cranien. - Rameau dorsal. - Le composant postérieur du vague est dirigé obliquement en arrière et latéralement. De son bord supérieur, à une petite distance du cerveau, part un filet nerveux très mince (fig, 3, rdv) qui s'élève dans la cavité cranienne, en allant en avant, au-dessous du plafond du crâne. Il chemine dans le tissu cellulo-adipeux qui entoure le cerveau, et plusieurs de ses ramifications s'anastomosent avec des filets dépendant du nerf dorsal du trijumeau-facial.

Stannius (16) pensait que le Silure, comme beaucoup d'autres Poissons qu'il cite, n'a pas de rameau dorsal du vague. Celui-ci existe bien, mais dans un état tout-à-fait rudimentaire, tandis que le rameau dorsal du trijumeau-facial a acquis une très grande importance. Le rameau intra-cranien du vague, qui, 
à défaut d'autres preuves, suffirait à montrer que le composant postérieur contient des éléments sensibles à côté des éléments moteurs, est donc un dernier vestige des rameaux dorsaux des nerfs segmentaux qui ont pris part à la formation du vague. E.-H. Weber (2 ; Pl. V, fig. 30) a représenté une ramification intra-cranienne de ce nerf, mais sans lui mettre une désignation quelconque. On remarquera qu'elle est plus forte que je ne l'ai indiqué et qu'en outre, elle prend naissance à une plus grande distance du cerveau, passablement plus distalement que la réunion des « trois racines ». Il n'y aurait rien d'étonnant à ce que ce filet nerveux, en voie de rétrogradation, fût soumis à des variạtions de position et de volume.

Je dois rappeler ici que le rameau de renforcement envoyé, d'après E.-H. WEBER, par la racine antérieure du vague au nerf auditif accessoire (cordon postérieur de l'acoustique v. p. 99) n'existe probablement pas. Par contre, il y a un faisceau communicant entre le composant antérieur du vague et le composant postérieur du glosso-pharyngien (fig. 3, 4).

Le composant antérieur, après un trajet d'environ $4 \mathrm{~mm}$., et le composant postérieur, après un trajet d'environ $2 \mathrm{~mm}$., s'appliquent l'un contre l'autre et sont accolés par du tissu conjonctif. Ainsi réunis, ils passent derrière l'ampoule du canal semi-circulaire postérieur et arrivent bientôt contre la paroi de la cavité cranienne. Pendant ce parcours commun, les deux composants sont d'abord situés l'un à côté de l'autre, puis, peu à peu, le composant antérieur se place dorsalement. On voit alors partir du composant postérieur un faisceau d'éléments peu considérable qui s'élève légèrement et pénètre dans le composant antérieur, à son bord inférieur. Ce fait avait été mentionné déjà par STANnius, mais cet auteur ajoute que le cordon de renforcement possède un ganglion distinct, ce que je ne puis pas confirmer. Il est très possible que ce cordon contienne des cellules ganglionnaires, mais il paraît conserver le même diamètre (3-4 mm.) dans toute sa longueur. 
Les composants du vague augmentant graduellement de volume en allant du cerveau vers la paroi du crâne, doivent certainement contenir tous deux des cellules ganglionnaires.

Sortie du crâne et division en branches. - Le-vague passe donc sur le plancher de la partie auditive de la cavité cranienne et arrive dans l'angle postéro-latéral de celle-ci. Le canal de l'occipital latéral, qui doit le conduire au dehors, débouche extérieurement par une ouverture largement évasée, qui occupe presque entièrement l'arête postérieure et inférieure du crâne. C'est pendant le passage à travers l'os que le cordon de renforcement partant du composant postérieur vient rejoindre le composant antérieur. Les deux composants continuent à augmenter de volume -- l'antérieur (le supérieur dans le canal) moins que le postérieur - et ils remplissent l'évasement qui termine le canal. Immédiatement après, ils se séparent; l'antérieur se prolonge en arrière et forme le nerf latéral du vague, le postérieur s'infléchit latéralement et constitue le nerf vague proprement dit ou nerf branchio-intestinal.

\section{A. Nerf vague proprement dit.}

Le nerf branchio-intestinal se divise immédiatement en un système de branches antérieures formant les troncs branchiaux et une forte branche postérieure, le tronc pharyngo-intestinal.

\section{Troncs branchiaux (Pl. 2, 3, fig. 3, 4, 7).}

Les troncs branchiaux qui sortent du renflement ganglionnaire formé par le composant postérieur du vague sont au nombre de trois. Ils se dirigent d'abord en avant, le long de la partie horizontale de l'occipital latéral et se séparent bientôt: le premier s'éloigṇe des autres dès sa sortie du tronc commun, les deux derniers restent unis sur un court trajet. Ces trois 
troncs possèdent chacun un ganglion distinct situé plus ou moins distalement. Le plus éloigné est le ganglion du tronc antérieur; ensuite, vient celui du deuxième et enfin celui du troisième qui se trouve à sa base même (fig. $3,4, g_{1-3}$ ). Ces ganglions ne sont pas très gros, mais ils sont très nettement délimités en avant et en arrière et leur forme est oblongue; le deuxième est un peu plus fort que les deux autres, ce qui correspond au volume plus considérable du deuxième tronc.

Les trois troncs s'infléchissent en avant en passant entre le rétracteur branchial antérieur et la partie supérieure (latérale) des obliques dorsaux situés au-dessous (Pl. 3, fig. 7).

En cet endroit, chacun d'eux émet, de son bord inférieur, un rameau (rod) dirigé médialement et en avant et qui pénètre dans les muscles obliques dorsaux par leur face supérieure. Ces rameaux appartiennent à ceux que STANNIUs a désignés comme rameaux pharyngiens supérieurs (p. 90). Le premier est fin et se rend au muscle antérieur. Le troisième est également faible; je ne puis dire s'il sort bien du troisième tronc branchial ou si c'est une ramification émise par le deuxième rameau, car dans la préparation il a été cassé près de sa naissance. Il va dans le muscle oblique inséré sur le quatrième arc branchial. Le deuxième est le plus fort des trois; il se divise en plusieurs filets avant d'entrer dans les muscles obliques insérés sur le deuxième et troisième arc. Quelques-uns de ses filets (fig. 8, rtd), se prolongent, passent entre les os pharyngo-branchiaux et parviennent dans le transverse dorsal. Il serait très possible que la partie du transverse dorsal qui relie l'extrémité supérieure du premier arc avec celle de son congénère, reçut un filet du premier rameau, mais je n'ai jamais pu en constater l'existence.

Le premier tronc branchial est celui qui a le plus long trajet entre le rétracteur du premier arc et les obliques dorsaux. Parvenu contre la face médiale des élévateurs branchiaux, il envoie 
un filet à celui de ces muscles qui s'insère sur le deuxième arc, et il se divise en une branche antérieure plus faible et une branche postérieure, plus forte.

La branche antérieure (Pl. 2, 3, fig. 4, 7, ba $\mathrm{T}_{1}$ ) continue à longer la face médiale des élévateurs, puis s'infléchissant latéralement, passe, en compagnie du glosso-pharyngien, entre les élévateurs des deux premiers arcs branchiaux. Elle arrive sur le premier arc dont elle constitue le nerf postérieur, tandis que le nerf antérieur est formé par le glosso-pharyngien. Notablement moins forte que ce dernier, elle suit le bord supérieur et postérieur de l'épibranchial; à partir du coude de l'arc, elle se place dans la rigole du cérato-branchial, au-dessous des rameaux du glosso-pharyngien, c'est-à-dire plus près de la périphérie. Elle va jusqu'à l'extrémité de l'arc, en longeant à sa base la rangée postérieure de lamelles branchiales. Les filets qui en sortent sont destinés, comme ceux du glosso-pharyngien, aux lamelles branchiales, aux vaisseaux sanguins qui courent dans le sillon médian et enfin à la muqueuse de l'arc.

La branche postérieure du premier tronc branchial (Pl. 2, 3, fig. $4,7, \mathrm{bp} \mathrm{T}_{1}$ ) est aussi volumineuse que le glosso-pharyngien. Elle s'infléchit latéralement pour passer entre l'élévateur du deuxième arc et l'élévateur du quatrième arc (fig. 7, Elb,$E l b_{4}$ ) ou bien, comme je l'ai observé une fois, à travers le premier de ces muscles, séparant ainsi un petit faisceau postérieur du reste du muscle. Ensuite, se repliant un peu en arrière, elle vient longer la rainure médiane du second arc. Arrivée à l'endroit où cet arc forme un coude, elle descend sur sa face antérieure et se rapproche du bord concave. Là, elle se bifurque; l'un des rameaux suit la courbure interne de l'arc jusqu'à son extrémité ventrale; il innerve les saillies dentiformes et la muqueuse environnante. L'autre rameau descend davantage et vient se loger dans la rigole du bord convexe de l'arc où il se comporte comme le glosso-pharyngien dans le premier arc: il s'y 
divise, et l'un de ses filets va jusqu'à la copule du deuxième arc. A l'endroit où la branche postérieure parvient sur l'épibranchial, elle émet un filet dont les ramifications s'étendent dans la muqueuse de la face antérieure, jusqu'au coude de l'arc.

Le premier trone branchial se divise donc en une branche antérieure, qui devient le nerf postérieur du premier arc branchial, et en une branche postérieure plus forte, qui va dans l'arc suivant dont elle forme le nerf antérieur. Les trones suivants se comportent de même vis-à-vis des autres arcs.

Le denxième tronc branchial est un peu plus. volumineux que les autres. Il se divise tout près de son ganglion, et les deux branches vont en avant sur une courte distance, puis s'infléchissent bientôt vers les arcs. La branche antérieure (ba $\mathrm{T}_{2}$ ) passe en arrière des élévateurs branchiaux et constitue le nerf postérieur du deuxième arc. Son trajet $y$ est analogue à celui du nerf postérieur du premier arc, mais, avant d'y pénétrer, elle émet un filet qui va jusqu'au coude de l'arc suivant et innerve la muqueuse de la face antérieure. La branche postérieure (bp $\mathrm{T}_{2}$ ) du deuxième tronc se dirige tout de suite latéralement. Elle atteint le troisième arc dont elle représente le nerf antérieur et au coude duquel elle se divise en un rameau supérieur et un rameau inférieur. Elle présente une particularité : à son entrée dans l'arc, elle n'émet pas de filet pour la face antérieure, celuici étant fourni, comme on vient de le dire, par la branche antérieure du même tronc.

Le troisième tronc branchial, dont le ganglion tient par la base au renflement ganglionnaire du tronc branchio-intestinal, est immédiatement divisé en deux branches qui s'éloignent aussitôt l'une de l'autre, ce qui peut les faire prendre pour deux troncs indépendants. La branche antérieure (ba $\mathrm{T}_{3}$ ) croise par devant le dilatateur branchial (ou rétracteur branchial postérieur, $R b p$, fig. 7), passe entre les extrémités latérales et postérieures des deux masses superposées des obliques dorsaux et 
livre alors un filet. Celui-ci descend sous la muqueuse, entre le troisième et le quatrième arc, se replie médialement vers la pièce pharyngienne supérieure et pénètre dans le muscle élévateur du quatrième arc. La branche envoie, en outre, un rameau dirigé en arrière, destiné à la muqueuse du quatrième arc. Elle forme le nerf postérieur du troisième arc et se comporte normalement durant son trajet. La branche postérieure (bp $\mathrm{T}_{3}$ ) se dirige en arrière et latéralement pour contourner par derrière le dilatateur branchíal, puis elle s'élève à la face postérieure du quatrième arc contre le constricteur du pharynx (fig. 7, Cph) et le prolongement de l'oblique dorsal (Cons IV). Elle ne parvient sur le bord convexe de l'arc que dans la deuxième moitié de l'épibranchial. A partir de ce point et jusqu'au coude, elle n'est pas située comme la branche postérieure des autres arcs sur le bord antérieur, mais sur le bord postérieur. Ce n'est qu'à partir de cette place qu'elle se rapproche de la rangée antérieure de lamelles et qu'elle prend un trajet normal. Cette branche m'a paru fournir un rameau au dilatateur branchial, au moment où elle passe contre sa face médiale, et un autre rameau assez fort à la partie du constricteur du pharynx qui s'insère sur le quatrième arc.

Le quatrième arc branchial reçoit un nerf postérieur (fig. 4, 7, nbp IV) qui ne provient pas de l'un des trois troncs branchiaux dont il a été question jusqu'ici, mais sort de la forte branche pharyngienne inférieure (phi) à l'endroit où celle-ci s'éloigne des autres branches du tronc pharyngo-intestinal. Ce nerf se dirige latéralement et un peu en avant, atteint le coude du quatrième arc, à sa face médiale (postérieure), en passant au-dessus de l'insertion du petit muscle qui relie l'extrémité postérieure de l'os pharyngien inférieur à l'extrémité distale du quatrième et dernier épibranchial. En ce point, il fournit dorsalement un rameau qui se replie dans la direction inverse de celle du nerf et se rapproche donc de l'extrémité médiale de l'épi- 
branchial, en suivant la rangée postérieure de lamelles branchiales. Enfin, le nerf parvient sur le quatrième cérato-branchial où il longe la rangée postérieure de lamelles.

On peut voir, d'après cet exposé, que les nerfs branchiaux se répartissent bien métamériquement selon le schéma indiqué par Gegenbaur $(31,32,35)$. Remarquons que, chez le Silure, les deux troncs branchiaux postérieurs tendent à se diviser chacun en deux branches, dès leur naissance, autrement dit qu'ils tendent à former chacun deux nerfs indépendants.

GEgenbaur a montré (35, p. 276) que la bifurcation des nerfs segmentaux en une branche destinée à un arc et une autre branche destinée à l'arc suivant s'explique par le fait qu'un arc se forme à la limite de deux métamères et que l'apparition de fentes branchiales sur ces métamères entraîne la division du nerf en deux branches, dont l'une va à la cloison antérieure, l'autre à la cloison postérieure. Quant à l'individualisation des deux branches ainsi formées, elle s'explique évidemment par des rapports topographiques particuliers, tels que la faible distance entre le tronc branchial et les arcs qu'il doit innerver et le développement de la musculature. En effet, chez le Silure, le premier tronc branchial est très long et ne se divise que lorsqu'il est parvenu en face des deux premiers arcs branchiaux. Le troisième tronc, au contraire, n'est représenté que par le ganglion, qui n'est pas même complètement distinct de la masse ganglionnaire du vague proprement dit. Des deux branches qu'il forme, la première peut pénétrer immédiatement dans le quatrième arc, tandis que la deuxième est fortement déviée vers l'arrière pour passer derrière le dilatateur branchial.

Des détails sur les rameaux livrés à la musculature des arcs par les trones branchiaux ont déjà été donnés à propos des muscles de l'appareil branchial. 


\section{Tronc pharyngo-intestinal.}

Ce tronc est formé de la partie postérieure du vague proprement dit. En sortant de la masse ganglionnaire de ce dernier, il présente un renflement moins distinct, quoique plus volumineux, que ceux des troncs branchiaux. Il se divise immédiatement en branches de grosseur différente qui restent étroitement accolées sur un trajet assez long.

Ces branches descendent contre la face médiale du dilatateur branchial, à peu près jusqu'au milieu de sa longueur et le croisent inférieurement pour se diriger latéralement. Jusqu ici, elles sont placées les unes derrière les autres, de manière à former une sorte de ruban aplati, large, avec des stries longitudinales. La plus forte de ces branches est celle qui occupe le bord postérieur et plutôt médial du ruban; elle devient la branche intestinale. Celle du bord antérieur et plutôt latéral est environ de la grosseur du premier tronc branchial; sa partie la plus importante constitue la branche pharyngienne inférieure. Enfin, la branche intermédiaire, peu volumineuse, donne naissance au rameau cardiaque et se distribue principalement à la musculature et à la muqueuse des os pharyngiens supérieurs; je la désigne comme branche pharyngo-cardiaque.

Avant de se diviser, le tronc pharyngo-intestinal envoie un rameau à l'élévateur claviculaire (v. p. 43). En poursuivant dans le tronc les fibres des trois branches, on reconnaît que ce rameau sort, en réalité, de la base de la branche pharyngienne inférieure. Il va latéralement et en arrière, et se place sous le nerf latéral ou bien entre ce dernier et le nerf occipital situé un peu en arrière. Il est alors accolé étroitement à l'aponévrose du muscle latéral et son trajet est difficile à suivre; souvent, on serait tenté de le prendre pour un rameau du nerf latéral ou encore pour un nerf indépendant qui naîtrait entre le vague et 
l'occipital. Arrivé vers la région moyenne de la barre osseuse allant du supra-claviculaire à la base du crâne, le rameau se dirige un peu en avant, traverse l'aponévrose du muscle latéral et arrive contre le bord médial de l'os claviculaire, à une petite distance de l'articulation avec le supra-claviculaire. Il pénètre, déjà divisé en deux ou trois filets, dans le muscle élévateur claviculaire par la face postérieure appliquée contre la clavicule.

Branche pharyngienne inférieure (Pl. 2, 3, fig. 3, 4, 10, phi). Cette branche a de nombreuses ramifications dirigées, comme la branche elle-même, en avant et en bas, parallèlement aux arcs branchiaux. Il y a pourtant deux fins rameaux qui font exception; ils naissent, très rapprochés l'un de l'autre, du bord interne de la branche, alors que celle-ci est encore accolée aux deux autres branches du tronc pharyngo-intestinal. Ces deux rameaux accompagnent la branche pharyngienne inférieure jusqu'au bord latéral supérieur du pharynx; là, ils s'infléchissent médialement et en avant pour se rendre dans la partie dorsale du constricteur du pharynx. Ils fonctionnent donc comme des rameaux pharyngiens supérieurs.

Un peu plus loin que l'origine de ces deux rameaux, naît un rameau plus important, qui descend d'abord le long de la branche, puis va horizontalement vers l'extérieur et atteint le coude du quatrième arc branchial dont il devient le nerf postérieur (v. p. 112).

Enfin, un autre rameau est émis par la branche pharyngienne inférieure. Il se dirige latéralement et passe derrière le petit muscle qui réunit l'extrémité postérieure de l'os pharyngien inférieur au coude du quatrième arc branchial. Le rameau envoie un filet à ce muscle et, de l'autre côté, un filet au constricteur pharyngien; puis il continue son parcours sur la face antérieure de l'arc pharyngien, dans la muqueuse duquel il se ramifie (fig. 3).

Après l'émission de ces différents rameaux, la branche pha- 
ryngienne, restée encore assez forte, s'avance à la face ventrale du pharynx, à une petite distance et médialement par rapport à l'os pharyngien inférieur. Elle se rapproche de cet os et se divise en deux rameaux destinés aux muscles pharyngo-claviculaires interne et externe (Pl. 3, fig. 10). Quelquefois, elle pénètre dans le muscle interne par son bord médial et vers le milieu de sa longueur; elle lui abandonne des filets en le traversant et entre ensuite dans le muscle externe (v. p. 40).

Pendant la partie ventrale de son parcours, la branche fournit encore plusieurs rameaux. Le premier est dirigé latéralement ; il innerve la muqueuse qui recouvre l'arc pharyngien du côté alvéolaire. Les autres sont dirigés médialement en avant. Il y en a toujours au moins trois, mais deux sont plus forts; ils se ramifient beaucoup, pénètrent dans le transverse pharyngien (fig. 10,Pht) et là, leurs extrémités ténues forment un réseau compliqué, dont quelques filets peuvent être suivis jusque dans la muqueuse du pharynx.

La branche pharyngienne inférieure pourrait être comptée comme un quatrième tronc branchial. Elle présente certains caractères qui permettent de lui attribuer cette valeur morphologique. En premier lieu, elle contient aussi bien des éléments sensibles que des éléments moteurs, puis son rameau antérieur devient le nerf postérieur du quatrième arc branchial, tandis qu'un autre de ses rameaux va dans l'arc pharyngien incomplet. Les différences, si évidentes soient-elles, qui existent entre le parcours dorsal des nerfs branchiaux antérieurs et celui des deux rameaux qui viennent d'être nommés, n'ont aucune importance; elles sont la conséquence de modifications secondaires, telles que le développement de la musculature dorsale des arcs branchiaux et la réduction de toute la partie supérieure de l'arc pharyngien. Des différences beaucoup plus importantes se montrent dans le trajet ventral, mais elles ne concernent que le rameau postérieur, l'antérieur se comportant tout à fait normalement comme nerf postérieur du quatrième arc. 
En effet, pour être conforme au schéma général des troncs branchiaux du vague, la branche pharyngienne inférieure, après avoir émis ses rameaux pharyngiens supérieurs et, plus loin, le nerf postérieur du quatrième arc, devrait constituer le fort nerf antérieur de l'arc suivant. Il n'en est pas ainsi; elle n'envoie à ce dernier qu'un faible rameau, tandis que la plus grande partie de ses éléments se continue comme branche presque exclusivement motrice destinée aux deux pharyngo-claviculaires et. au transverse pharyngien. Cette anomalie n'est encore qu'une modification secondaire. L'arc pharyngien ayant perdu toute fonction respiratoire, son nerf branchial ne peut contenir des fibres nerveuses destinées aux lamelles branchiales et doit se réduire considérablement. Mais, l'arc réduit a reçu d'autres attributions; il a un rôle actif dans l'acte de la déglutition. Son extrémité inférieure est tirée en bas, en arrière et latéralement par deux muscles puissants, les pharyngo-claviculaires, tandis que le transverse pharyngien le ramène en avant et médialement. Il n'est donc pas étonnant que la partie motrice de la branche soit assez considérable et qu'elle se soit séparée du petit contingent d'éléments sensibles destiné à la muqueuse de l'arc. Nous ne pouvons voir autre chose dans la branche pharyngienne inférieure, qu'un tronc branchial adapté très étroitement aux fonctions actuelles de l'arc pharyngien, lesquelles fonctions ont certainement été acquises très anciennement.

Branche pharyngo-cardiaque. - Cette branche est, de beaucoup, la moins volumineuse de celles qui sortent du tronc pharyngo-intestinal. Elle envoie une partie de ses éléments à la muqueuse des os pharyngiens supérieurs et à la musculature dorsale du pharynx. D'après cela, on pourrait l'appeler branche pharyngienne supérieure, mais cette désignation aurait l'inconvénient de lui prêter une valeur morphologique autre et plus importante que celle qu'elle semble avoir. En effet, elle n'est pas seule à innerver la région dorsale du pharynx; elle partage ce domaine 
avec des nerfs provenant des troncs branchiaux et de la branche pharyngienne inférieure. En outre, la branche pharyngo-cardiaque n'est pas exclusivement destinée au pharynx, elle émet un rameau assez fort destiné au cœur. Les filets qu'elle envoie au pharynx ne sont probablement réunis que secondairement à la branche cardiaque et ont la même signification par rapport au tronc pharyngo-intestinal que les rameaux pharyngiens supérieurs précédents par rapport aux troncs branchiaux.

La branche pharyngo-cardiaque se divise en trois parties avant d'atteindre le pharynx, c'est-à-dire pendant son trajet commun avec les deux autres branches du tronc pharyngo-intestinal. Deux des rameaux s'infléchissent médialement et en avant, pénètrent dans la région dorsale du constricteur pharyngien auquel ils abandonnent plusieurs ramifications. Le second de ces rameaux est le plus fort; il peut être suivi jusqu'à la face inférieure des os pharyngiens supérieurs dans la muqueuse desquels il se résout en de nombreux filets.

Le troisième rameau de la branche pharyngo-cardiaque est le rameau cardiaque (Pl. 2, fig. 3, 4, rcd). D'après Stannius, Weber (Anatom. comp. N. symp. p. 63) n'aurait vu qu'une partie de ce rameau chez Lucioperca. G. Büchner (9) a trouvé que chez Cyprinus barbus, il est émis par la branche intestinale et qu'il est très fin; il l'a suivi jusque dans l'oreillette. STANNIUs (16, p. 91) l'a observé chez Perca, Cottus, Cyclopterus, Belone, Gadus, Esox, Salmo, Cyprinus, Alosa, Acipenser, Spinax. Voici ce qu'il en dit: "Dieser bemerkenswerthe Zweig ist sehr fein, tritt von einem $R$. pharyngeus oder $R$ cesophagus ab und begleitet den Ductus Cuvieri seiner Seite zum Vorhofe des Herzens. » C.-E.-E, Hoffmand (21, pp. 8 à 10) dans sa courte recherche anatomique sur le vague des Poissons, donne une description détaillée du rameau cardiaque chez Cyprinus carpis, Perca fluviatilis, Barbus fluviatilis, Leuciscus vulgaris et rutilus, Esox lucius, Gadus lota et morrhua. Il indique le trajet 
et l'endroit précis où se terminent la plupart des rameaux et constate la présence de corps ganglionnaires bipolaires dans plusieurs de ces rameaux. Cette recherche minutieuse était faite dans le but d'étudier les mouvements du cœur.

Chez le Silure, le rameau cardiaque semble être une ramification d'un «rameau pharyngien », ce qui correspond aux indications de Stannius rappelées ci-dessus. Il nous semble plus juste de le considérer, avec BüchNER, comme un rameau de la branche intestinale, qui se serait séparé de celle-ci dès sa naissance et se serait uni, sur un court trajet, avec les rameaux que le tronc pharyngo-intestinal livre au pharynx. Le rameau descend sur la face latérale du tube digestif, dans l'angle formé par la branche intestinale et la branche pharyngienne inférieure, parallèlement à cette dernière. Il atteint la partie postérieure et ventrale du sinus veineux, passe près de la base du canal de Cuvier et arrive sur la face dorsale de l'oreillette dans laquelle il se termine.

Branche intestinale (Pl, 2, 3, fig. 3, 4, 10, bi). C'est la plus forte des branches du tronc pharyngo-intestinal. Lorsqu'elle est parvenue au niveau supérieur du pharynx, elle se dirige en arrière, le long de l'œsophage, en se plaçant dans le repli mésentérique latéral. Il faut indiquer ici que l'œsophage n'est pas soutenu par un mésentère dorsal médian, mais par deux replis latéraux du péritoine, qui atteignent l'œsophage de chaque côté, plutôt dorsalement. La présence de ces deux replis latéraux au lieu d'un repli sagittal est probablement une conséquence de l'extension de la vessie natatoire vers l'avant, caractère commun aux Ostariophyses. Ce double mésentère ne s'étend que le long de l'œsophage; le reste du tube digestif est maintenu par un mésentère dorsal médian.

La branche intestinale longe donc latéralement l'œsophage et parvient sur la paroi de l'estomac dans laquelle elle s'épanouit en de nombreuses et fortes ramifications. Pendant son trajet le 
long de l'œsophage, elle émet de nombreux rameaux. La plupart de ceux-ci se dirigent ventralement et médialement sur la paroi du canal; seuls, les antérieurs vont en avant, vers le pharynx. Leurs ramifications sont très nombreuses et sont enchevêtrées dans la couche musculaire, mais des filets terminaux vont également dans la muqueuse. En outre, la branche intestinale émet de son bord dorsal un rameau qui va en arrière et en haut et se répand dans la paroi de la vessie natatoire.

Les rapports entre la branche intestinale et le nerf splanchnique ont été exactement décrits par Stannius : les deux nerfs se côtoient sans se confondre, mais de fréquentes unions ont lieu entre leurs ramifications.

\section{B. Nerf latéral du vague.}

(Pl. 2, fig. 3, 4, nlv)

Le nerf latéral du vague, chez les Poissons, a été l'objet d'un nombre considérable de recherches anatomiques et physiologiques. D'après celles-ci, le nerf latéral présente plusieurs types, soit sous le rapport de sa composition et de ses relations avec les autres nerfs, soit sous le rapport du nombre et du trajet de ses rameaux, soit encore sous le rapport de sa situation et de ses relations avec la ligne latérale. Il ne sera rappelé ici que les indications des auteurs ayant un intérêt général, puis nous examinerons le trajet et la division en branches du nerf latéral du Silure.

Cuvier et Valenciennes (7, p. 527) disent : «Dans beaucoup de Poissons, notamment dans la Perche, après avoir donné un filet superficiel qui suit le commencement de la ligne latérale, ce nerf marche en ligne droite dans l'épaisseur des muscles latéraux, entre les côtes et leurs appendices, recevant de tous les nerfs de l'épine des filets particuliers différents des intercos- 
taux, et en donnant à la peau, au travers de tous les interstices des couches musculaires. »

Büchner (9, p. 27) confirme l'indication de l'union du nerf latéral avec les nerf spinaux. «L'anastomose est fine et a lieu avec la branche superficielle des nerfs spinaux ».

Schlemm et D'Alton (10) décrivent le nerf latéral des Pétromyzons. Ce nerf est formé par des éléments des deux racines du vague et par une branche du facial. Il s'unit avec le rameau supérieur du premier nerf cervical et touche les rameaux supérieurs des autres nerfs cervicaux sans se fusionner avec eux.

D'après Prevost (14), le nerf latéral du Congre est divisé en deux faisceaux parallèles qui ne s'unissent que rarement par une ou deux anastomoses. Le faisceau interne envoie quelques petits filets nerveux vers la ligne médiane et la colonne vertébrale. Le faisceau externe émet des filets destinés aux pores de la ligne latérale; «ils se subdivisent souvent sur leur chemin et s'anastomosent probablement quelquefois avec les nerfs rachidiens. »

Stannius $(16$, p. 92 à 110) a montré différents états sous lesquels le nerf latéral des Poissons peut se présenter. Un certain nombre de ses indications, dont quelques-unes concernent spécialement le Silure, ne sont pas d'accord avec ce que j'ai pu observer; aussi la description qui va suivre insistera-t-elle surtout sur ces points. Cet auteur nie le passage d'éléments des nerfs spinaux dans le tronc même du nerf latéral.

Dans ses «Recherches sur le système latéral du nerf pneumogastrique des Poissons », Félix FÉE (29) reconnait trois types principaux qui se rattachent aux différentes positions que le canal latéral peut occuper par rapport au plan de séparation des deux masses musculaires des flancs. Il n'admet pas non plus le renforcement du tronc latéral par les nerfs spinaux et n'affirme même pas qu'il y ait de véritables anastomoses entre ces derniers et les rameaux ventraux de ce tronc. 
C. Gegenbaur (35) regarde le nerf latéral comme un ou plusieurs rameaux dorsaux ayant acquis une grande extension dans un domaine étranger à la partie céphalique du corps.

Suivant Beadregard (56), le nerf latéral du Ceratodus est formé d'une partie du vague renforcée par un rameau du facial.

Dans «The Lateral Line System in Siluroids», PoLLARD (86) ne s'occupe que de la partie céphalique ${ }^{1}$. Pour ce qui concerne le nerf latéral du vague, il ne parle donc que du premier rameau «muqueux», le rameau surtemporal, qui innerve le dernier organe sensitif cranien du système latéral. Une commissure part de ce rameau pour rejoindre dans le pariétal le "grand rameau récurrent dorsal du facial» (nerf de WEBER). Disons tout de suite que cette commissure n'a été trouvée ni par Stannius, ni par moi chez Silurus glanis et que sur les cinq Siluroïdes qu'il a examinés ( $S$. glanis n'en fait pas partie), Pollard l'a vue chez tous, sauf chez Trichomycterus tenuis.

Le nerf latéral du Silure sort de la moelle allongée par un unique faisceau désigné plus haut comme composant antérieur du vague. Il se dirige latéralement en arrière et vient s'appliquer contre le bord antérieur du composant postérieur du vague. Il commence à former un ganglion qui est alors assez considérable en diamètre dorso-ventral, mais très peu en diamètre transversal. Le composant du nerf latéral s'élevant un peu, se place alors sur le composant du vague proprement dit, et reçoit de ce dernier un petit faisceau d'éléments (v. p. 107). Ensuite, il atteint la paroi du crâne, passe à travers l'occipital latéral par le même orifice que l'autre composant. Le volume continue à augmenter considérablement et cela provient sûre-

1 Il a déjà été question de ce travail à propos du nerf de Weber. Voir pour plus de détails aux p. วั6, כั7 et 58 . 
ment davantage de la grande accumulation de cellules ganglionnaires que de la réception du faisceau de renforcement. Ce dernier ne peut être qu'une petite fraction des éléments sensibles (dorsaux) réunis à la racine ventrale du nerf vague pour constituer le composant postérieur.

En sortant du crâne, le nerf latéral se place sur l'aponévrose qui relie la portion dorsale du muscle latéral du tronc à l'arête inféro-postérieure du crâne neural. Il continue à aller latéralement et en arrière, en passant d'abord derrière l'extrémité supérieure du dilatateur branchial, puis au-dessus du premier nerf spinal (Pl. 2, fig. 4). Il n'est séparé de ce muscle et de ce nerf que par l'aponévrose dont il vient d'être question.

A une très petite distance du trou de l'oceipital latéral, le nerf latéral émet dorsalement un rameau qui suit l'arête inféropostérieure du crâne dans une petite rainure de l'exoccipital. Ce rameau s'infléchit latéralement pour traverser l'arête osseuse non loin de son bord et se poursuivre à la face inférieure de l'exoccipital, puis du squameux au bord latéral duquel il vient émerger. Là, il se divise en deux parties. L'une va en bas et en avant entre les muscles élévateur et adducteur de l'opercule; elle s'accole temporairement au rameau operculaire du tronc hyoïdeo-mandibulaire et se rend dans la muqueuse de la face interne de l'opercule, sans avoir livré de filets aux muscles operculaires qu'elle traverse. Cette partie semble être la seule que Stannius aît observée. L'autre partie descend sur la face latérale de l'élévateur operculaire (Pl. 3 fig. 5, rov) et se ramifie dans la peau qui recouvre extérieurement l'opercule et le rayon branchiostège élargi remplaçant un sous-opercule.

Ce premier rameau est donc le rameau operculaire du vague (fig. $3,4,5$, rov). Le nerf latéral émet un peu plus loin un autre rameau dorsal qui est sans doute l'analogue du rameau suprascapulaire de Stannius $(16$, p. 97 ) et du rameau surtemporal de Pollard; mais je préfère le nommer rameau supra-clavi- 
culaire (fig. 3, 4, scl) d'après l'os sur lequel il se rend en partie'. En ontre il faut remarquer que Stannius décrit (p. 96) les rameaux operculaire et supra-scapulaire comme ramifications d'une même branche; je n'ai jamais observé une telle disposition.

En effet, lorsque le nerf latéral donne naissance à son second rameau dorsal, il est déjà parvenu dans le voisinage de la pointe postérieure de l'exoccipital. Le rameau s'élève sur l'aponévrose du muscle latéral (portion dorsale), puis sur la partie principale de l'os supra-claviculaire, soit celle qui se fixe sur l'exoccipital. Là, il envoie un filet au canal muqueux qui est situé dans le supraclaviculaire et qui communique en arrière avec le canal de la ligne latérale du tronc. Arrivé sur la face supérieure de l'os, le reste du rameau s'y résout en plusieurs ramifications destinées à la peau de cette région, sauf l'une d'elles qui va un peu plus loin en arrière.

Stannius a donné (16,p. 106 et 107$)$, pour le reste du trajet du nerf latéral chez le Silure, une description, qui, à part plusieurs points qu'elle laisse obscurs, présente des divergences évidentes avec ce que j'ai observé. Par exemple, Stannius dit que le tronc latéral "traverse sous la ceinture scapulaire " et cette indication semble avoir été répétée par d'autres auteurs, sans aucun contrôle. Pour le Poisson qui nous occupe, c'est certainement une erreur et on comprend facilement qu'elle n'est pas sans importance, par exemple pour la signification de la branche nerveuse.

Le nerf latéral, après avoir donné les deux rameaux décrits, les seuls qui se distribuent dans la région céphalique, continue à aller à peu près horizontalement en arrière. Il est toujours situé sur l'aponévrose de la masse dorsale du muscle latéral et

${ }^{1}$ Le nom de supra-scapulaire a été appliqué, postérieurement à STanvius, à un os neltement déterminé chez certains Poissons et existant à côté d'une autre pièce, le supra-claviculaire. C'est l'homologue de ce dernier que nous trouvons chez le Silure (voir Sagemehl, Cranium der Characiniden, 1885̈). 
croise dorsalement la partie antérieure de la pièce qui doit être regardée comme l'arc inférieur (apophyse transverse) de la deuxième vertèbre. Cette partie antérieure ne parait être qu'une apophyse devenue excessivement puissante en raison de sa fonction comme appui de la ceinture scapulaire; elle dépasse de beaucoup, en volume, la partie postérieure. Cette dernière représente l'arc inférieur proprement dit, c'est une simple pointe osseuse dirigée en arrière, semblable quant à la forme aux arcs inférieurs des vertèbres suivantes (Pl. 1, fig. 2, $\mathrm{Ai}_{2}$ ). Le nerf croise l'apophyse antérieure près de son extrémité latérale qui est réunie à la partie postérieure du supraclaviculaire par de courts ligaments. Les modifications des pièces squelettiques de cette région sont très nombreuses, elles se rapportent toutes à l'appareil de WEBER et compliquent beaucoup l'explication du trajet des nerfs. Malgré cela, on ne peut se rendre compte de l'erreur de STAnNius qu'en supposant qu'il n'a pas suivi le nerf latéral dans toute sa longueur. Ce nerf reste situé médialement par rapport à la ceinture scapulaire. Il croise un os qui se relie à celle-ci, mais ne lui appartient pas, car c'est une dépendance de la deuxième vertèbre. Enfin il passe au-dessus et non au-dessous de cet os.

Pour faciliter l'exposé du trajet dans le tronc et dans la queue, il est nécessaire de donner quelques renseignements préliminaires sur la disposition de la musculature dans ces régions.

On sait que de chaque côté du corps se trouve un muscle latéral divisé en métamères par des myocommes et que ce muscle comprend une portion supérieure ou dorsale et une portion inférieure ou ventrale (schéma, p. 133 md, mv). Les auteurs parlent fréquemment de l'une ou de l'autre de ces portions, mais il est rare que l'on indique exactement ce qui leur sert de limite. Chez le Silure, tout au moins, cela n'est pas clair. En effet, lorsqu'on enlève la peau sur l'un des côtés du corps, on peut voir une ligne légèrement sinueuse, mais très accentuée, 
commençant au point de jonction du claviculaire et du supraclaviculaire. Elle longe le flanc, d'abord à mi-hauteur, exactement en face de l'extrémité des côtes, puis se rapproche de la face ventrale vers la région anale. Enfin, dans la région caudale qui représente à peu près les deux tiers de la longueur totale, elle se trouve au niveau de l'extrémité des apophyses inférieures, c'est-à-dire au niveau de l'union de celles-ci avec les osselets interépineux ou rayons porteurs de la nageoire anale. Cette ligne est la limite extérieure d'un interstice très faible renfermant les côtes et la mince aponévrose qui les relie (schéma, p. 133, ap); je l'appellerai ligne des côtes.

Au-dessus de cette première ligne s'en trouve une autre, dont le commencement s'observe un peu en arrière de la tête et qui s'accentue peu à peu d'avant en arrière. Elle descend légèrement et se relève ensuite sur la plus grande partie du tronc et sur la région caudale. Sur une section transversale du tronc, on voit que cette ligne est la limite d'un interstice (schéma, p. 133 , ii) bien distinct, qui commence le long de la colonne vertébrale, au-dessus de l'origine des côtes, et s'étend latéralement en présentant une courbure à concavité supérieure. C'est cet interstice que je regarde comme limite des masses dorsale et ventrale du muscle latéral.

En considérant seulement la partie antérieure du tronc, on serait plutôt tenté de considérer la ligne des côtes comme ligne de séparation, car elle est beaucoup mieux marquée extérieurement et elle commence immédiatement derrière la ceinture scapulaire. En outre, la portion musculaire située entre ces deux lignes - peu considérable en avant, mais très importante en arrière - ne peut être délimitée en avant d'avec la portion dorsale; elle constitue avec elle une masse qui s'insère sur l'os supra-claviculaire et la partie postérieure du crâne. Enfin, la direction des fibres dans les myomères est sensiblement la même dans la région supérieure à la ligne de séparation et dans la 
région située entre la ligne de séparation et la ligne des côtes ; cette direction tend à former avec celle des fibres situées audessous des côtes un V couché dont la pointe serait tournée en arrière.

La forme des myocommes ne peut être invoquée en faveur ni de l'une, ni de l'autre des alternatives. En effet, au-dessus de la ligne de séparation, leurs limites périphériques sont obliques de haut en bas et d'avant en arrière; au-dessous de la ligne des côtes, elles sont obliques dans le sens inverse, et dans la partie comprise entre les deux lignes, les limites se raccordent avec celles de dessus et de dessous, en formant une ligne sinueuse rappelant une sorte de $\mathrm{V}$ à pointe tournée en arrière et à branches très écartées.

Nous avons examiné les caractères d'après lesquels il semblerait juste de considérer la ligne des côtes comme ligne de séparation des deux masses musculaires du tronc. Quels sont ceux qui militent en faveur de l'alternative opposée? En premier lieu, la présence d'un gros sinus lymphatique, tout le long de la ligne de séparation, caractère indiqué par F. FÉE (29). Puis le fait que la ligne de séparation devient très accentuée en arrière et prend l'aspect d'un sillon longitudinal. Une section transversale dans la région caudale montre, à ce niveau, un espace triangulaire, rempli par une bande musculaire de couleur gris brun, non divisée en segments et semblable aux bandes ventrale et dorsale trouvées par Cuvier chez la Perche et présentes aussi chez le Silure. Dans cette même région, enfin, la ligne reste toujours au niveau de la colonne vertébrale, ce qui fait que les deux masses musculaires ont à peu près un volume égal. Si, au contraire, la ligne des côtes devait être la ligne de séparation, ce serait la portion dorsale qui formerait la musculature presque entière de la queue, tandis que la portion ventrale serait réduite aux faisceaux longeant les rayons porteurs de la nageoire anale. 
Nous reconnaissons donc la ligne supérieure comme ligne de séparation des masses dorsale et ventrale du muscle latéral. Celle-ci ne commence à être visible qu'à quelque distance de la tête, parce qu'une partie de la masse ventrale se joint à la masse dorsale, de telle manière que toute trace extérieure de séparation disparaît. La masse musculaire commune ainsi formée passe au-dessus de l'extrémité supérieure de l'os claviculaire et se fixe sur la face supérieure de la tête. La portion dorsale proprement dite s'attache, par une large aponévrose et par divers cordons tendineux, à la crête inféro-postérieure du crâne formée par l'occipital latéral et l'exoccipital, à la face supérieure du squameux, du pariétal, de la partie postérieure du frontal principal, sur le supra-occipital et sa crête sagittale; la petite partie de la masse ventrale qui est jointe à la portion dorsale se fixe à la face dorsale du supra-claviculaire et, plus en avant, sur la région latérale du squameux. L'extension de cette partie sur la face supérieure du crâne ne peut être que secondaire; elle est du reste très peu importante si on regarde le supraclaviculaire comme une pièce de la ceinture scapulaire. On n'est donc pas bien loin de pouvoir dire que la portion dorsale du muscle latéral s'insère en avant sur le crâne et la portion ventrale sur la ceinture scapulaire. Mais l'insertion ne peut servir de critère pour délimiter les deux portions l'une de l'autre, et l'emploi exclusif de ce caractère pourrait, dans des cas particuliers, comme celui du Silure, conduire à de grosses erreurs.

Il est nécessaire de donner encore quelques explications sur la ligne latérale de Silurus glanis. Extérieurement, celle-ci est fort peu visible, car, comme on le sait, les écailles manquent complètement. Il faut même avoir soin d'enlever la couche de mucus qui recouvre la peau pour apercevoir la ligne mince et très légèrement en relief. Elle correspond à un canal longitudinal (schéma, p. 133, cl) logé dans le derme et dont le dia nètre ne semble pas dépasser $0,5 \mathrm{~mm}$. 
Ce fin canal commence à l'extrémité postérieure du supraclaviculaire et entre en communication, par le canal renfermé dans cet os, avec les canaux des os de la tête. Il est alors situé au dessus de la ligne des côtes et au-dessous de la ligne de séparation des deux masses musculaires, même plus près de la première que de la seconde. Il se trouve au niveau de cette dernière, lorsqu'elle devient nettement visible. Ceci, soit dit en passant, permet de penser que la ligne de séparation devrait commencer au même endroit que la ligne latérale du tronc, mais qu'elle disparait dans cette région parce que la masse musculaire ventrale, ayant crû davantage à la périphérie qu'à l'intérieur, a redressé un peu le bord de la masse dorsale et a même recouvert latéralement une partie de cette masse.

Le canal latéral reste vis-à-vis de la ligne de séparation, donc à peu près à mi-hauteur sur le flanc, jusqu'à la nageoire caudale. Un peu au-dessous du canal latéral, on observe de petites éminences cylindriques de la peau (schéma, p. 133, e), percées d'un canalicule qui débouche dans le canal latéral. Ces éminences, très courtes, ressemblent plutôt à de petits boutons et sont dirigées obliquement de haut en bas et d'avant en arrière. Dans la partie antérieure du tronc, elles sont distantes de 3 à $5 \mathrm{~mm}$. du canal latéral, mais vers l'arrière, elles s'en rapprochent insensiblement et à l'extrémité de la queue, elles sont au même niveau que lui. Enfin, leur disposition est nettement segmentale, chacune d'elles se trouvant en face ou près d'un myocomme; rarement il s'en trouve deux très rapprochées et, dans ce cas, on peut voir qu'il s'agit de la division d'une éminence primitivement unique.

Après cette parenthèse, nous reprenons l'examen du nerf latéral à partir de l'endroit où il arrive sur l'arc inférieur de la deuxième vertèbre.

Le nerf latéral, encore très volumineux, s'infléchit maintenant en arrière et s'introduit dans l'interstice entre les 
masses dorsale et ventrale du muscle latéral (schéma, p. 133, md, mv). Dans le commencement du tronc proprement dit, il est situé plus près de la colonne vertébrale que de la périphérie, un peu au-dessus des côtes, soit dans la partie horizontale de l'interstice (schéma, nlv). En allant en arrière, il se rapproche de la périphérie, si bien que dans la région caudale, il est logé au fond du sillon à section triangulaire occupé par la bande musculaire non-segmentée. Il diminue graduellement d'avant en arrière, mais on peut le suivre jusqu'à l'extrémité postérieure du corps. Là, le sillon latéral s'atténuant jusqu'à s'annuler, le nerf devient tout à fait superficiel et se divise en deux rameaux dont les ramifications se répaudent dans la peau de la nageoire caudale.

Durant ce trajet, le nerf latéral ne se trouve pas constamment sous la forme d'un faisceau unique. Un peu en arrière de la ceinture scapulaire, il est régulièrement divisé en deux parties qui restent toutes deux dans l'interstice, mais s'éloignent l'une de l'autre. L'interne, par son volume et par sa direction, peut être regardée comme le prolongement du tronc. L'externe ne devient jamais superficielle; en arrière, elle se rapproche du faisceau interne, peut même quelquefois le rejoindre, puis s'en éloigne de nouveau et s'en rapproche encore; elle se fusionne définitivement avec lui dans la région avoisinant les vertèbres VIII à X. Des rameaux communicants, sortant irrégulièrement du faisceau interne, viennent renforcer le faisceau externe et forment généralement entre les deux un réseau compliqué. On observe très souvent des variations individuelles ou même des différences entre les deux côtés d'un même animal, soit sous le rapport des points de bifurcation et de réunion des deux faisceaux, soit dans leur volume et leur éloignement, soit encore dans le nombre et la force des filets anastomotiques. (La division en deux faisceaux n'a pas été représentée dans les figures 2 et 3 , afin de ne pas compliquer davantage les dessins). 
Voyons maintenant quelles sont les branches livrées par le nerf latéral après les rameaux operculaire et supra-claviculaire qui ont été examinés précédemment.

Il y a d'abord, pendant le passage au-dessus de l'arc inférieur de la deuxième vertèbre, un dernier rameau dorsal qui contourne l'extrémité postérieure de l'os supra-claviculaire et se rend dans la peau de cette région.

La ramification suivante, que je désigne comme branche superficielle du nerf latéral, est une branche assez forte (fig. 3, 4 , bs), dont le diamètre peut atteindre $1,5 \mathrm{~mm}$; elle naît du bord ventral du tronc, un peu plus en arrière que le rameau qui vient d'être décrit. Quelquefois sa naissance se trouve beaucoup plus en avant et alors la branche peut avoir un trajet différent; je citerai un cas exceptionnel de ce genre, après avoir montré l'état qui paraît être le plus fréquent.

la branche superficielle se dirige d'abord médialement et parallèlement au supra-claviculaire et vient se placer au niveau des côtes, à mi-distance entre leur extrémité distale et la périphérie; puis, obliquant légèrement, elle vient affleurer au niveau de la ligne des côtes, exactement à la limite entre le deuxième et le troisième myomère. A partir de ce point, la branche (schéma, p. 133, bs) court entre la masse ventrale du muscle latéral et la peau, à une petite distance de la ligne des côtes; elle se rapproche done rapidement de la face ventrale du corps et se termine toujours avant d'atteindre la région caudale. Elle fournit elle-même plusieurs rameaux, dont le premier (Pl. 2, fig. 3, 4, $\mathrm{r}_{i}$ ), livré en face de l'extrémité postérieure du supra-claviculaire, descend sous la peau parallèlement au bord postérieur de l'os claviculaire et se perd dans le tégument vers la base de la nageoire pectorale. Ce premier rameau arrive à la périphérie du muscle exactement à l'endroit où commence la ligne des côtes et descend le long du premier segment musculaire du tronc. Le second rameau $\left(r_{2}\right)$ est aussi 
émis pendant le trajet à travers le muscle latéral; il vient affleurer à la ligne des côtes, dans le second myomère sur le flanc duquel il descend. A partir de là, la branche devenue superficielle livre au niveau de chaque myomère un rameau qui est d'autant plus petit qu'il appartient à un segment plus postérieur (schéma, p. 133, rbs). La branche superficielle du nerf latéral innerve donc la peau des flancs au-dessous de la ligne des côtes.

Les indications de Stannius au sujet de cette branche sont très sommaires, mais je pense que les deux rameaux qu'il a vus sortir du tronc latéral et se rendre à la peau du membre antérieur et à la peau de la région ventrale ne sont que les deux premiers rameaux de la branche superficielle. J'ai toujours vu ces deux rameaux naitre de la branche superficielle, mais STANNIUS a peut-être observé un cas remarquable de variation individuelle, dans lequel le point où la branche superficielle se détache du tronc latéral se trouverait repoussé loin en arrière; les deux premiers rameaux de la branche devraient alors se détacher directement du tronc pour pouvoir se rendre à leurs domaines respectifs, la région du premier et du second myomère.

L'existence d'une telle disposition ne paraît pas impossible si l'on sait que le point de séparation de la branche superficielle a une situation assez variable. Ce ne serait du reste qu'un cas extrême, opposé à celui que je puis relater ici. La branche superficielle se séparait du nerf latéral à une petite distance du passage de celui-ci à travers l'occipital latéral, presque en face de l'origine du rameau operculaire. Elle se plaçait sur l'aponévrose de la masse musculaire dorsale et s'élevait un peu audessus du nerf latéral, en suivant de près l'arête inféro-postérieure du crâne; puis elle croisait le nerf latéralement à peu près à l'endroit où elle nait ordinairement. Jusque là, il y avait entre le nerf latéral et la branche superficielle quelques commu- 
nications par de petits filets qui, suivant leur direction, établissaient un passage d'éléments tantôt dans un sens, tantôt dans l'autre. Plus distalement, la branche se comportait d'une manière normale en émettant ses rameaux ventraux, y compris les deux destinés au premier et au second métamère. L'état le plus fréquent serait donc intermédiaire entre ce dernier et celui qui a été décrit par StanNiUs.

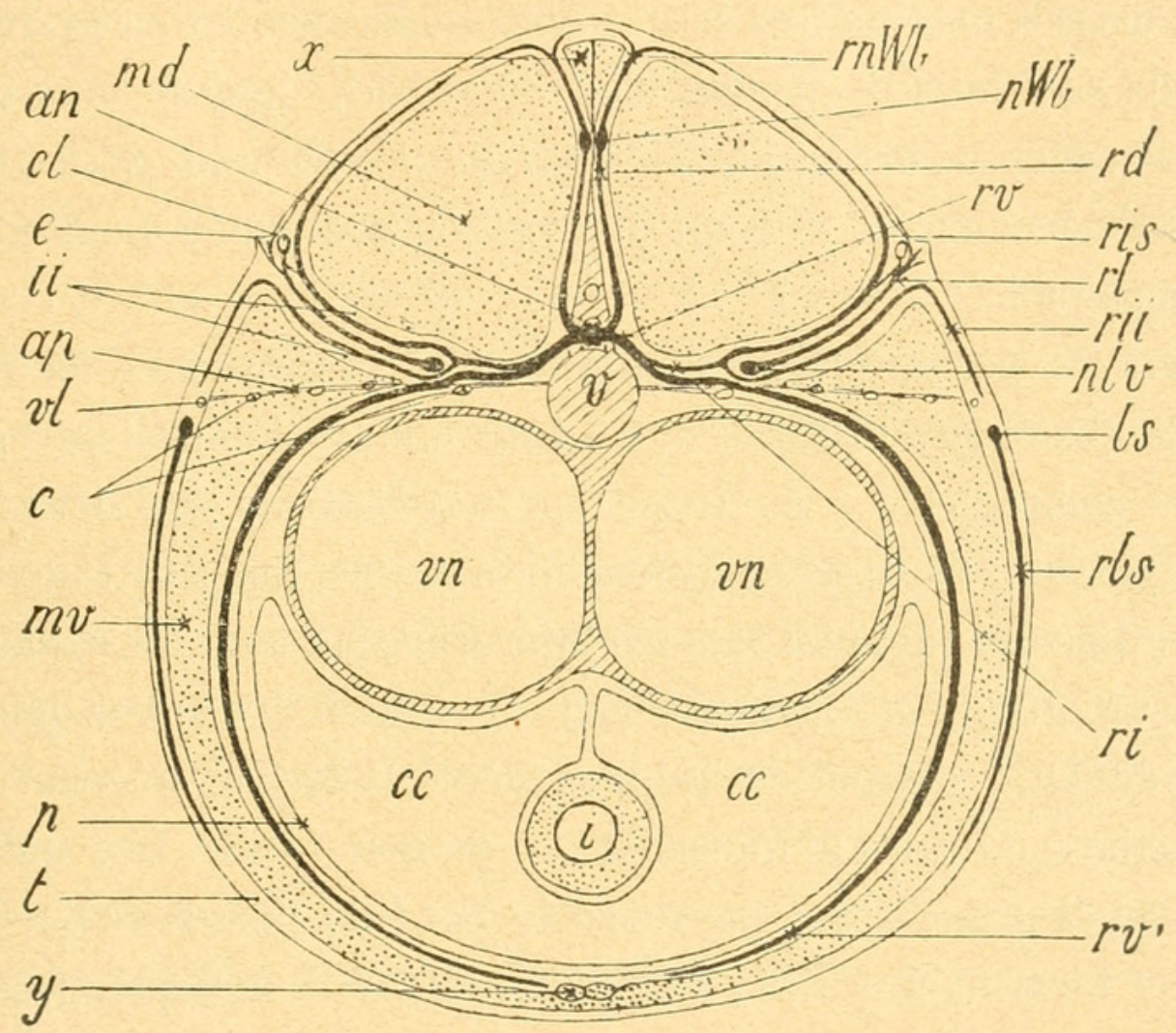

Schéma représentant une section transversale faite dans la région antérieure du tronc du Silurus glanis. $v$, vertèbre. an, arc neural. $c$, côtes. $t$, tégument. $c c$, cavité du corps. $p$, peritoine. $i$, intestin. $v n$, vessie natatoire. mid, masse dorsale du muscle latèral. $m v$, masse ventrale. $x, y$, bandes musculaires dorsale et ventrale, ap, aponévrose intercostale. $v l$, vaisseau lymphatique, $i i$, interstice intermusculaire. $c l$, canal latéral. $e$, éminence de la ligne laté- rale. $r v$, ramean ventral d'un nerf spinal. $r v^{\prime}$, prolongement principal de $r v, r i$, ramean intermédiaire issu de $r v$. ris, rii, rameaux supérieur et inférieur de $r i$. $n l v$, nerf lateral du vague. $r l$, rameau intersticiel de $n l v . \quad b s$, branche superficielle de $n l v . r b s$, rameau ventral de $b s$. $r d$, rumeau dorsal d'un nerf spinal. $n W b$, nerf de WEBER. $r n W b$, rameau de $n W b$ destiné à la peau de la région dorsale.

Les autres ramifications du nerf latéral sortent toutes latéralement; ce sont des rameaux très fins, livrés assez régulièrement à raison de un par métamère (schéma, p. 133, rl). Chacun d'eux s'avance dans l'interstice entre les deux masses musculaires, en 
suivant de près le myocomme postérieur du segment auquel il appartient. Dans ce trajet, il est accompagné par un rameau également grêle (ri) qui se détache de la branche ventrale de chaque nerf spinal non loin du canal neural. Le rameau ventral (rv) du nerf spinal traverse le fascia qui recouvre les côtes (rameau intercostal) et se prolonge sur la face interne du muscle ventro-latéral tandis que le rameau ( $R$. medius Stannius, R. intermédiaire F. FÉE) qu'il a émis reste dans l'interstice des deux masses musculaires et se divise généralement en arrivant près du nerf latéral en deux filets très fins, dont le supérieur (ris) - plus rarement l'inférieur (rii) — envoie un petit faisceau d'éléments au rameau du nerf latéral que les deux filets accompagnent.

Vers la périphérie de l'interstice, le rameau issu du nerf latéral (ou rameau latéral) et les deux filets du rameau intermédiaire commençent à se diviser en ramifications très délicates qui s'entre-croisent et se fusionnent souvent. On peut toujours suivre l'un des filets du rameau latéral jusque dans le canal de la ligne latérale et, généralement, on réussit à trouver que ce filet, ou plus souvent le rameau dont il dérive, a reçu une anastomose du rameau intermédiaire du nerf spinal. Les autres filets du rameau latéral, parvenus au bord de l'interstice, s'élèvent et forment un réseau très riche et très compliqué sous la peau qui revêt la masse dorsale du muscle latéral. Ils partagent d'ailleurs ce domaine d'innervation avec le filet supérieur du rameau intermédiaire, tandis que le filet inférieur du même rameau se ramifie dans la peau recouvrant la masse musculaire ventrale. Dans la région où le nerf latéral est divisé en deux faisceaux, c'est le faisceau externe (latéral) qui livre les rameaux de l'interstice.

Pour ce qui concerne la question du renforcement du tronc latéral par des éléments des nerfs spinaux, je pense que les auteurs qui ne l'admettent pas ont raison (voir la revue de 
bibliographie, p. 131 à 132). L'erreur de Cuvier et de BüchneR doit être surtout attribuée, comme l'indique FÉE, à la bifurcation du rameau intermédiaire près du tronc latéral. Les relations qui existent entre le nerf latéral et les nerfs spinaux n'ont lieu qu'à la périphérie et seulement entre de fines ramifications.

Nous avons constaté que, chez Silurus glanis, les rameaux livrés par le tronc latéral pendant son passage dans l'interstice intermusculaire envoient un filet au canal latéral, puis se ramifient dans la peau qui recouvre les métamères correspondants. Cet état est, sans doute, plus primitif que celui qui a été interprété exactement pour la première fois par BAUDELOT (38, p. 135) et dans lequel les rameaux s'infléchissent en arrière et soudent leurs extrémités. De cette manière, il se forme une «branche superficielle » qui court à côté du canal latéral et se trouve reliée au tronc latéral par des rameaux communicants (p. ex. chez la Perche).

Stannius ignorait la véritable origine d'une telle branche que l'on pourrait très bien appeler fausse branche superficielle. De même, il semble n'avoir pas connu la continuité qui existe entre le canal latéral du tronc et le système de canaux contenu dans le couvercle cranien, l'opercule et la mâchoire inférieure. C'est sans doute de là que provient « la confusion dans la partie de son travail concernant le système latéral du vague » (FÉE, p. 34).

Pour ce qui concerne spécialement le Silure, Stannius dit à la page $99:$ «les plus fortes branches du nerf latéral sont dépourvues de relations quelconques avec le canal latéral ", puis plus loin (p. 106): «il se sépare (du tronc latéral) une branche longitudinale superficielle, qui descend d'avant en arrière, inférieurement au canal latéral ... tandis que le tronc latéral passant entre les deux masses du muscle latéral «émet des filets qui, longeant les ligaments intermusculaires, vont de la profondeur à la périphérie... où ils se répandent généralement sous la peau». 
D'après ces trois passages, il est facile de trouver ce qui a causé des divergences entre les indications de STANnIUs et la description donnée ci-dessus : $1{ }^{\circ}$ Stannius a considéré comme canal latéral un vaisseau lymphatique qui court à la superficie du tronc, le long de l'aponévrose reliant les côtes à la peau, soit le long de la ligne des côtes (schéma, p. 133, vl); c'est en effet un peu au-dessous de celle-ci que passe la branche superficielle (bs) du nerf latéral; $2^{\circ}$ il n'a pas eu connaissance de ces petites éminences (e) en forme de papilles, rangées en une série segmentale le long de la ligne de séparation des masses musculaires dorsale et ventrale et qui ne s'aperçoivent que très difficilement sur un animal mort, si on ne râcle pas le mucus blanchâtre recouvrant la peau; $3^{\circ}$ il semble avoir regardé la ligne des côtes comme ligne de séparation des deux masses musculaires. Stannius indique, il est vrai, que le tronc latéral passe entre les deux masses musculaires, mais ceci ne diminue pas la valeur de notre supposition; en effet, dans la région antérieure du corps, le tronc latéral se trouve situé à l'intersection de l'aponévrose intercostale et de l'interstice intermusculaire (comme dans le schéma, p. 133) ou un peu plus latéralement, soit alors dans la partie médiale de l'interstice.

Nous comprenons maintenant pourquoi Stannius dit que les plus fortes branches sont sans relation avec le canal latéral, tandis que réellement le tronc latéral lui-même (la plus forte branche) innerve ce canal par des ramifications de ses filets latéraux. Quant à la branche superficielle, il serait bien étonnant que, passant si près du prétendu canal latéral, elle ne lui envoyât pas de nerfs, surtout si elle était l'homologue de la branche superficielle de la Perche par exemple, comme StanNius l'a pensé.

Ceci nous amène à examiner quelle est la signification de la branche superficielle du Silure. Il est facile de reconnaître qu'elle n'est pas comparable à celle qui se rencontre chez beaucoup 
de Poissons (Percoïdes, par ex.) et que l'on pourrait appeler fausse branche superficielle. En effet, une fausse branche superficielle est toujours produite par la réunion des rameaux du tronc latéral. Au contraire, la branche superficielle du Silure semble devoir être considérée comme formée par les deux premiers de ces rameaux dont la séparation d'avec le tronc latéral a été reportée du côté de la tête, tandis que leurs ramifications ont gagné une grande extension dans la région ventrale située audessous de la ligne des côtes. A la grandeur du domaine d'innervation correspond naturellement un énorme développement des rameaux. Le déplacement du point de séparation vers l'avant, de telle manière que les deux premiers rameaux semblent être deux ramifications d'une branche issue du tronc latéral, offre évidemment l'avantage de rapprocher plus rapidement les nerfs de la région à innerver. Les deux rameaux envoient chacun un filet à la partie du canal latéral située sur les deux premiers segments musculaires, (on se souvient que dans cette région le canal latéral est voisin de la ligne des côtes), puis quelques ramifications à la peau environnante. Ce filet et ces ramifications correspondent donc à chacun des rameaux suivants du nerf latéral. Le prolongement commun des deux rameaux et les branches ventrales émises au niveau de chaque myomère du tronc proprement dit ne sont que le résultat d'une extension secondaire.

La conception de la branche superficielle du Silure comme résultat d'un grand développement des deux premiers rameaux latéraux est renforcée par l'observation de cas exceptionnels tels que celui décrit comme normal par STANNiUs, cas dans lequel la branche superficielle ne livrerait pas les rameaux destinés aux deux premiers segments, ceux-ci étant fournis directement par le tronc latéral. Un tel état est dû simplement, comme nous l'avons déjà dit, au fait que la branche reste plus longtemps en connexion avec le tronc latéral. L'objection qui se 
baserait sur le fait que la branche naît ventralement tandis que les autres rameaux sortent latéralement du nerf latéral ne semble pas avoir de valeur si on remarque que la branche superficielle est devenue une branche destinée surtout à la région ventrale, tandis que les autres rameaux continuent à innerver un domaine situé au même niveau que le tronc latéral et même plus élevé.

Le système latéral du Silure qui, d'après les auteurs, semblait difficilement réductible à un type général, s'y rattache donc d'une manière facile et complète. La conséquence de ces considérations est que le nerf latéral possède une unité de plan beaucoup plus grande que les anciens auteurs ne le laissent supposer. BAUdELot (38), FÉE (29) et d'autres anatomistes ont déjà fait la lumière sur différents points et les recherches futures pourront peut-être donner des résultats analogues pour beaucoup d'autres cas.

On sait depuis longtemps que le nerf latéral ne doit avoir aucune propriété motrice(v. Stannius, J. Muller, C. E. HofFMANN, FÉE, etc). L'idée que le canal latéral serait un organe destiné à sécréter une mucosité et que le nerf latéral présiderait à cette fonction a été combattue avec autorité par LEYDIG (17) et bien d'autres après lui. D'après FÉE : «Si le nerf latéral exerce une influence quelconque sur la sécrétion du mucus, c'est par ses branches cutanées et non pas par celles qui se distribuent au prétendu canal muqueux. » Son action sur l'acte respiratoire semble encore problématique.

Ce qui paraît certain pour le moment, c'est que le nerf latéral est un nerf de sensibilité générale en relation avec des organes des sens particuliers, situés dans le canal latéral. Cela suffit pour comprendre comment il se fait que le Silure possède un tronc latéral très volumineux, tandis que le canal latéral est très faible, et pour faire repousser l'idée émise par FÉE (29) qu'il y ait généralement proportionnalité entre le nerf et le 
canal. Si le canal est fortement développé, on conçoit que le nerf doive être plus volumineux, mais la réciproque n'est pas toujours vraie. Ainsi, chez le Silure, et probablement chez les autres Poissons dont la peau nue peut percevoir facilement les sensations, un fort canal latéral n'est pas nécessaire. Le nerf envoie la majeure partie de ses éléments dans la peau ellemême; il conserve donc la même importance et peut même en acquérir une plus grande, tandis que le canal se réduit.

Quand à la signification morphologique du nerf latéral, l'interprétation avancée par BAUDELOT (38) et appuyée par FÉE, semble satisfaire le mieux aux exigences de la critique et des connaissances actuelles. Je le considère donc aussi comme homologue d'un rameau intermédiaire de nerf spinal, ou peut-être plus exactement, d'une réunion de rameaux intermédiaires. Cette opinion est basée sur les observations et les réflexions persuasives des deux auteurs nommés et sur le fait que, chez le Silure, les rameaux intersticiels du nerf latéral partagent certainement leur domaine d'innervation (peau du flanc et canal latéral) avec les rameaux intermédiaires des nerfs spinaux.

\section{Nerf occipital.}

Le dernier nerf cranien des Poissons, appelé souvent hypoglosse, a été l'objet d'interprétations diverses. Nous passerons en revue les principales opinions, non pas dans l'ordre chronologique, ce qui serait très long et fastidieux, mais en groupant autant que possible les auteurs d'après l'affinité de leurs conceptions. Ceci permettra d'examiner de plus près les travaux récents qui ont placé la question sur un terrain plus solide.

Beaucoup d'anciens auteurs disent simplement que l'hypoglosse manque complètement chez les Poissons, par exemple : Desmoulins (1822), Longet (1842) et Girgensohn (1846). 
Baddelot ( $† 1875$, publication posthume 1883$)$, ne parle pas non plus d'un nerf cranien postérieur au vague, et rien dans ses observations sur les premiers nerfs spinaux ou sur le vague ne montre qu'il se soit préoccupé de chercher si une branche de ceux-ci pouvait en tenir lieu.

D'autres constatent qu'il y a encore un nerf sortant du crâne après le vague et le considèrent comme un nerf cérébral particulier, toutefois sans le regarder comme hypoglosse; de ce nombre sont Weber (1820), Cuvier et Valeṇciennes (1828), Pretost (1846). Leurs indications sur le trajet périphérique sont, comme celles de beaucoup d'autres auteurs du reste, ou complètement nulles ou très incomplètes.

Parmi les anatomistes qui ont accordé un hypoglosse aux Poissons, il faut citer: Büchner (1835), Schlemm et d'ALTON (1840), Cuvier (Anat. comp. T. III, 1845), OWEN (1866, d'ap. Fürbringer 1897), Beauregard (1881), Jaquet (Anat. comp. de Vogt et Yung, T. II, 1894).

BücHNER est parmi ces auteurs celui qui donne les indications les plus exactes sur la répartition de ce nerf. De même que Cuvier, il dit qu'il se divise en deux branches, dont l'antérieure innerve les muscles des os pharyngiens et le sterno-hyö̈dien (Cuvier : muscles coraco-hyoüdiens et muscles de l'hyoüde), tandis que la postérieure, unie au premier nerf spinal, va dans les muscles de la nageoire pectorale.

D'après Stannius (1849), le nerf en question n'est que le premier spinal naissant par deux racines, une antérieure et une postérieure, qui sortent souvent ensemble par un trou de l'occipital latéral. Ce nerf pouvant être intervertébral et livrant régulièrement des éléments au plexus brachial, STAnnius ne le considère pas comme hypoglosse, et ajoute (p. 124): "vielmehr wird letzterer Nerv bei den Fischen nur durch einen Ast des ersten Spinalnerven repräsentirt. ”

L'hypoglosse serait représenté chez les Poissons par le ou 
les premiers nerfs spinaux, telle est l'opinion de GEGENBAUR (Anat. comp. 1870, trad. fr. 1874) et de Wiedersheiv (Anat. comp. 1895). VETTER (1878), admet que l'innervation du sterno-hyoüdien a lieu par le rameau antérieur des deux premiers nerfs spinaux réunis. Chez Acipenser ruthenus, GoRonowitsh (1888) trouve trois nerfs entre le premier spinal et le vague; ce sont des parties de racines ventrales de nerfs spinaux. Le premier s'unit, après la sortie du crâne, avec les branches ventrales des trois premiers nerfs spinaux; la distribution des deux derniers n'est pas connue.

En 1871, GEgENBAur admet que les racines naissant chez les Sélaciens au-dessous et en arrière du vague sont des " racines ventrales du vague ». Chez les Poissons osseux, celles-ci sont réduites, rapprochées et unies à des racines dorsales du vague pour former l'accessoire de Weber, nerf qui, chez les Vertébrés supérieurs, deviendrait l'hypoglosse et l'accessoire de WiLlis. Schneider (1878), Wiedersheim (1880), Ahlbohrn (1884), d'après des recherches sur Petromyzon et Ammocoetes, admettent aussi que l'hypoglosse est une partie du groupe du vague.

SAGEMEHL appelle nerfs occipitaux des nerfs devenus craniens par suite de l'adjonction de vertèbres au crâne primordial, le dernier nerf du crâne primordial étant le vague. Chez les Sélaciens, il n'y a pas de nerfs occipitaux; ils n'apparaissent que chez les Poissons à squelette ossifié, car c'est chez eux que l'assimilation de vertèbres au crâne commence à se montrer. Chez Amia calva (1883), il doit y avoir au moins trois vertèbres ajoutées au crâne primordial: la première a son corps soudé à l'occipital basilaire et son arc supérieur à l'occipital latéral; le nerf qui lui correspond sort donc par un trou de l'occipital latéral. Les corps des deux autres vertèbres sont soudiés aussi au basilaire; leurs arcs supérieurs ne se sont pas fusionnés avec le crâne et sont désignés comme arcs occipitaux libres. Le second nerf occipital sort donc entre l'arc soudé 
au crâne et le premier arc libre, et le troisième nerf occipal entre les deux arcs libres. - Chez les Characinides (1885), qui sont des Ostariophyses, il y a un seul nerf occipital qui naît par une forte racine ventrale et une racine dorsale très faible, formant un petit ganglion intra-cranien. Ce seul nerf occipital correspond au deuxième de Amia; il sort de l'occipital latéral, car chez les Téléostéens, le deuxième arc occinital est toujours soudé au crâne. Les Poissons osseux possèdent souvent un deuxième nerf correspondant au troisième de Amia, mais son état est variable et dépend du sort du dernier arc occipital auquel il correspond. Ce dernier, chez les Ostariophyses, est transformé en claustrum et la mobilité de cet osselet de l'appareil de WEBER contre l'occipital latéral motive la disparition du nerf. - Dans un troisième travail (crâne des Cyprinoüdes, 1891), SAGEMEHL a exposé, en passant, les différents cas qui se présentent chez les Poissons osseux sous le rapport du dernier arc occipital

En 1888, Gegenbaur ${ }^{1}$ reconnait que les soi-disant * racines ventrales du vague » des Sélaciens n'appartiennent réellement pas au vague et qu'elles ne sont parvenues que secondairement dans le domaine de la tête. Le nerf des Téléostéens interprété comme hypoglosse a la même signification. Ces cordons nerveux offrent des variations dans leur origine et leur composition; de plus, ils s'unissent avec des nerfs spinaux pour innerver une région comprenant le domaine de l'hypoglosse plus un domaine étranger à celui-ci; donc "nous ne pouvons attribuer la signification d'un hypoglosse ni à ces racines inférieures du vague, ni aux nerfs spinaux qui leur sont réunis et encore moins aux deux ensemble. On ne peut dire que ceci : l'hypoglosse se sépare seulement de ces nerfs ».

1 Gegenbaur résume les indications de Ahrborn, Schneider, Wiedersheim sur les Cyclostomes, de Stannius, Jackson et Clarke, Vetter, Onodi sur les Sélaciens. Voir cet ouvrage pour ces indications qui ne pouvaient trouver place ici. 
M. FüRBRINGER (1897) ${ }^{1}$ admet aussi que les prétendues racines du vague des Sélaciens n'ont rien à faire avec le vague. Ce sont des nerfs spinaux incorporés depuis longtemps au crâne et il les appelle nerfs occipitaux. Des nerfs spinaux incorporés plus récemment leur font suite, ce sont des occipito-spinaux; l'ensemble constitue les spino-occipitaux. Chez les Poissons osseux, il n'y a que des occipito-spinaux (nerfs occipitaux de SAGEMEHL), les occipitaux ont complètement disparu. Amia a trois nerfs occipito-spinaux $a, b, c$, les Téléostéens n'en ont que deux $b, c$, dont le sort varie avec celui des ares occipitaux auxquels ils correspondent. Hors du crâne, ces nerfs forment avec le premier nerf spinal un complexe cervico-brachial, dont la faible partie antérieure (plexus cervical) innerve la musculature spinale hypobranchiale, et la partie postérieure (plexus brachial ou ptérygial), la nageoire pectorale. Le plexus cervical est un rameau formé surtout par une partie de $b$, ou quand $c$ existe par une partie de $b$ plus une partie de $c$, cette dernière étant toujours la moins forte; il innerve les pharyngo-claviculaires interne et externe (cleido-branchialis 5 internus, cleido-blanchialis 5 externus), et se termine dans le sterno-hyoïdien (coracohyoideus s. cleido-hyoideus).

Mac Murrich (1885) n'admet pas comme SagemeHL que des vertèbres se sont fusionnées avec le crâne chez les Poissons osseux, mais il admet simplement que des corps de vertèbres se seraient soudés au basi-occipital, tandis que leurs arcs auraient disparu; les nerfs qui leur correspondaient se seraient réunis en un premier nerf spinal. C'est seulement chez les Amniotes que deux ou trois vertèbres se seraient soudées au crâne et que leurs nerfs réunis formeraient un véritable nerf cranien, l'hypoglosse. Cet auteur a trouvé que les deux pharyngo-claviculaires sont innervés par son premier nerf spinal.

1 Voir dans cet ouvrage la récapitulation des résultats de Fritsch (1878), Sanders (1887), Mayser (1881), Mc Murrich (1884) et R. Whrigt (1884). 
D'après HALLER (1897), le nombre des prétendues racines ventrales du vague peut se réduire de 5 à 1 . Le seul nerf qui persiste chez les Ganoïdes, les Téléostéens et les Dipnoïques est désigné comme nerf postvagal. Chez Salmo, Esox, il est formé seulement de deux racines antérieures et s'unit aux deux premiers nerfs spinaux pour innerver la nageoire pectorale; la région correspondant au domaine de l'hypoglosse ne reçoit pas de nerfs de lui, mais bien d'une branche du vague. Chez Cyprinus carpio, le postvagal, uni à des éléments venant du trijumeau et du vague, devient un accessoire de Weber qui se divise en deux branches. La postérieure entre en relation avec le premier nerf spinal et actionne la musculature de la nageoire pectorale, l'antérieure va dans la région innervée chez Salmo par la branche hypoglosse du vague. Cette dernière branche manque complètement à Cyprinus carpio. On peut donc dire que chez Salmo, ni les deux premiers nerfs spinaux, ni le postvagal n'ont un rapport quelconque avec l'hypoglosse des Vertébrés supérieurs. Chez Cyprinus, le postvagal qui tendait à s'avancer vers le vague et la partie hypoglosse du vague qui tendait à s'en séparer et à s'éloigner vers l'arrière se sont réunis en un accessoire de WEBER. Dans son recul, la partie hypoglosse a entraîné avec elle la portio du ganglion spinal du vague lui appartenant, ce qui explique la présence d'une racine dorsale dans l'accessoire de WEBER. L'étude histologique des origines confirme cette manière de voir, d'où l'on peut conclure que, chez les Poissons, l'hypoglosse provient toujours d'une partie du vague.

La question de l'origine de l'hypoglosse est donc encore en controverse, l'ingénieuse explication de HALLER demandant à être confirmée par l'étude d'un plus grand nombre d'espèces. Le seul résultat définitif de toutes ces recherches est que, chez les Poissons, il n'existe pas de nerf cranien indépendant qui puisse être regardé comme hypoglosse.

Chez le Silure, le dernier nerf cranien sort de la moelle 
allongée par deux racines, l'une supérieure munie d'un ganglion, l'autre inférieure, située ordinairement un peu plus en avant que la supérieure. La distance entre ces racines et celles du vague est généralement peu considérable, elle présente cependant des variations assez importantes qui ne sont pas toujours proportionnelles à la taille des individus et sont quelquefois même très sensibles entre les deux côtés d'un même animal. Voici les mesures qui ont été prises sur trois exemplaires dont le premier mesurait $60 \mathrm{~cm}$. environ, le second $90 \mathrm{~cm}$. et le troisième $1^{\mathrm{m}} 40$. Toutes les longueurs sont des distances horizontales et longitudinales, elles ont été mesurées à plusieurs reprises et ce sont les moyennes des résultats qui figurent dans le tableau. La longueur du cerveau est comptée depuis le bord antérieur du prosencéphale jusqu'au bord postérieur de la fosse rhomboïdale, soit par conséquent jusqu'au niveau du composant postérieur du vague; les distances entre ce dernier et l'une des racines du nerf occipital sont prises du bord postérieur au bord antérieur.

\begin{tabular}{|c|c|c|c|c|c|}
\hline & \multirow[t]{2}{*}{$\begin{array}{l}\text { LONGUEUR } \\
\text { DU CERVEAU }\end{array}$} & \multicolumn{2}{|c|}{$\begin{array}{l}\text { Distance du composant } \\
\text { posterieur du vague } \\
\text { à la racine dorsale du } \\
\text { nerf occipital. }\end{array}$} & \multicolumn{2}{|c|}{$\begin{array}{l}\text { Distance du composant } \\
\text { postérieur du vague } \\
\text { à la racine ventrale du } \\
\text { nerf occipital. }\end{array}$} \\
\hline & & Côté gauche & Côtẻ droit & Côté gauche & Côté droit \\
\hline & $\mathrm{mm}$ & $\mathrm{mm}$ & $\mathrm{mm}$ & $\mathrm{mm}$ & $\mathrm{mm}$ \\
\hline fer $^{\mathrm{e}}$ exemplaire. & $1 \check{, 00}$ & 2,00 & 2,00 & 1,23 & 1,25 \\
\hline $2^{\text {me }}$ exemplaire. & 18,60 & $1,7 \check{3}$ & 3, & 2,00 & 2, \\
\hline $3^{\text {me }}$ exemplaire. & 20,כั0 & 1,00 & $\breve{8}, 00$ & 1,23 & 4,30 \\
\hline
\end{tabular}

On voit, d'après ce tableau, que les points où les racines du nerf occipital émergent de la moelle allongée sont assez variables. Cependant, ces chiffres, malgré le soin que l'on peut mettre à les obtenir, n'ont qu'une valeur très relative, par le fait que les racines sont d'abord dirigées d'avant en arrière et qu'elles 
sont alors appliquées contre la moelle. Il est donc assez difficile de dire à quel point exact elles se séparent réellement du cerveau, ceci surtout pour la racine dorsale qui est toujours très grêle et que l'on voit quelquefois se prolonger dans la direction du composant postérieur du vague, sans pourtant faire saillie sur la moelle. Mais, même en tenant compte des différences provenant de ce fait, on peut être certain qu'il y a des variations notables et réelles d'un individu à un autre et entre les deux côtés chez un même individu.

La racine ventrale, dont le diamètre ne semble pas dépasser $1 \mathrm{~mm}$., sort nettement du cordon inférieur de la moelle, soit sur sa face ventrale près du bord inféro-latéral. La racine dorsale est toujours beaucoup plus faible que la racine ventrale; elle est généralement plus courte puisqu'elle naît ordinairement plus en arrière et sort de la face latérale de la moelle à mihauteur, ou légèrement au-dessus. Elle longe d'abord le bulbe d'avant en arrière, puis s'en éloigne bientôt et se trouve alors au-dessus de la racine ventrale où elle forme un ganglion ovoüde.

Le ganglion (Pl. 2, fig. 3, 4, g o) a un diamètre transversal plus fort que la racine ventrale; il est formé à une petite distance du cerveau, puisque son bord médial touche la face latérale de la moelle (dans la fig. 4, il en a été éloigné un peu, à dessein). Inférieurement, il repose sur la racine ventrale et, à son extrémité distale, il est soudé avec elle. Sur le côté droit, le ganglion se trouvait à $3 \mathrm{~mm}$. en arrière du composant postérieur du vague chez le premier exemplaire, et à $8 \mathrm{~mm}$. chez le troisième.

Le nerf constitué par la réunion des deux racines se dirige obliquement en arrière et latéralement sur le plancher de la cavité cranienne, mais son trajet y est toujours court (2 à $3 \mathrm{~mm}$.) Il atteint la base de la paroi du crâne formée par l'occipital latéral et traverse cet os par un canal particulier situé en 
arrière et légèrement plus bas que celui du vague. La distance entre les orifices internes des canaux du vague et de l'occipital varie sensiblement dans les mêmes proportions que la distance entre le vague et le ganglion du nerf occipital, tandis que la distance entre les orifices externes (en moyenne 2 à $3 \mathrm{~mm}$.) ne varie pas au delà de ce que la taille des individus permet d'admettre comme normal. L'orifice externe (fig. 1, oc) se trouve donc un peu en arrière de celui du vague, soit à environ $5 \mathrm{~mm}$. au-dessus de la barre osseuse (B Sc) qui part du supraclaviculaire et se fixe sur la base du crâne.

Après la sortie du crâne, le nerf (fig. 3, 4, oc) placé contre la face inférieure de l'aponévrose du muscle dorso-latéral, s'en va latéralement et un peu en arrière, en suivant de près le bord supérieur de la barre osseuse qui vient d'être mentionnée et se trouve alors très rapproché, souvent même en contact avec le fin rameau que le vague envoie au muscle occipitoclaviculaire. Le nerf chemine d'abord horizontalement, puis il s'infléchit vers le bas et croise par devant la barre osseuse du supra-claviculaire, à peu près à son tiers médial, soit plus près du crâne que le point où le nerf latéral passe au-dessus de la même barre. Jusque là, le nerf occipital n'émet qu'un fin filet ventral qui sort non loin de l'orifice de l'occipital latéral; ce filet est peut-être en communication avec le système sympathique, mais je n'ai pas pu m'en assurer exactement. Je n'ai pas trouvé de rameau dorsal.

Au moment où il descend devant la barre osseuse du supraclaviculaire, le nerf occipital entre en connexion avec le premier nerf spinal. Celui-ci est très faible; sa branche ventrale (Iv) vient descendre aussi sur la face antérieure de la barre osseuse et s'applique contre le bord supérieur du nerf occipital. Les deux nerfs sont réunis par du tissu conjonctif sur une longueur de plus d'un centimètre et, au premier abord, il semble que la fusion soit complète. Mais, du bord dorsal du ruban aplati résul- 
tant de cette union, part un filet nerveux qui longe inférieurement la barre osseuse et va se jeter dans le tronçon principal de la branche ventrale du deuxième nerf spinal. Si on débarrasse le ruban nerveux du tissu conjonctif, on voit qu'en réalité, le premier nerf spinal se divise en deux parties: l'inférieure, très courte, s'unit au nerf occipital; l'autre, accolée encore à ce dernier pendant un court trajet, s'en sépare et se joint au deuxième nerf spinal (fig. 3). Les deux parties semblent être de même force; s'il y a une différence, c'est plutôt à l'avantage de la première. Continuant à descendre tout en se dirigeant latéralement, le nerf occipital quitte la barre osseuse et se place sur la partie de la membrane péritonéale s'étendant plus en avant que la vessie natatoire. Il émet alors un rameau assez fort qui s'en va horizontalement et latéralement et passe entre le claviculaire et l'élévateur de cet os; parvenu au bord latéral de la clavicule, ce rameau s'infléchit en arrière et se ramifie dans la peau, près de l'articulation de la nageoire pectorale (fig. 3, 4).

Le nerf occipital, toujours situé sur la membrane péritonéale, descend maintenant verticalement, à peu près à égale distance entre la paroi latérale de l'œsophage et le bord médial de la clavicule. Au moment de s'infléchir pour prendre un cours ventral par rapport au pharynx, il se bifurque.

La partie postérieure est quelquefois un peu moins forte que l'autre; elle descend sur la paroi de la cavité du corps, en obliquant en arrière, s'engage entre le muscle ventro-latéral et la clavicule et s'unit avec le fort tronçon antérieur du deuxième nerf spinal (branche ventrale) lequel a reçu plus haut la communication du premier nerf spinal.

La partie antérieure (rameau hypoglosse, rhpg, fig. 3, 4, 10) se dirige en avant et en bas et parvient sur la face ventrale du pharynx. Là, elle repose directement sur l'enveloppe péricardiaque et s'avance entre le bord médial de l'os pharyngien infé- 
rieur et le bord latéral du ductus Cuvieri. Plus loin, elle s'infléchit latéralement et inférieurement, passe au-dessous du pharyngo-claviculaire interne, puis contre la face médiale du pharyngo-claviculaire externe. Elle atteint enfin le muscle longitudinal reliant la partie inférieure de la ceinture scapulaire à l'os hyoïdien, le muscle sterno-hyoïdien (Pl. 3, fig. 10, Sth).

A l'intérieur du sterno-hyoïdien, la branche nerveuse encore assez forte se divise en plusieurs |rameaux et quelques-uns de ses filets se prolongent ventralement jusque dans la peau. Sur tout le reste de son parcours ventral, elle ne livre aucun rameau et, par conséquent, n'actionne pas les muscles pharyngo-claviculaire comme FüRBRINGER et MAC MurRich l'ont trouvé chez d'autres Poissons. Ces muscles sont d'ailleurs sûrement innervés par un rameau provenant de la branche pharyngienne inférieure du vague.

Si maintenant nous comparons ce que nous avons dit du nerf occipital du Silure aux indications de la littérature, nous devons d'abord examiner le fait que ce nerf possède deux racines, une ventrale plus forte et une dorsale plus faible, munie d'un ganglion. Une telle origine fait immédiatement penser à un nerf spinal dont la racine dorsale a subi une certaine réduction. Cependant, d'après les recherches de HaLLer (101) sur la Carpe, il se pourrait que nous eussions affaire à un accessoire de WEBER. Dans ce cas, le composant ventral serait formé par les racines ventrales de nerf spinaux réduits et incorporés au crâne et par la portion hypoglosse du vague; le composant dorsal serait la partie des éléments dorsaux du vague jointe à la portion hypoglosse et qui aurait suivi celle-ci dans sa migration en arrière. Le Silure aurait done un accessoire de WEBER qui ne se distinguerait de celui de la Carpe que par l'absence de rameaux communicants du trijumeau et du vague.

La recherche des centres d'origine éluciderait peut-être complètement la question. Sans prétendre la trancher par d'autres 
moyens, il me semble que deux faits s'élèvent contre cette manière de voir. En premier lieu, la distance assez considérable qui peut exister entre les racines du vague et celles du nerf occipital; en deuxième lieu, le fait que la distance entre les orifices de sortie dı vague et du nerf occipital est toujours faible et à peu près la même, tandis que la distance entre les points où ces nerfs sortent de la moelle peut augmenter considérablement. Il semble alors plus naturel de regarder le nerf occipital comme un nerf spinal incorporé au crâne, et dont la racine dorsale est en voie d'atrophie.

Pour ce qui concerne les rapports du nerf occipital avec le premier nerf spinal, je pense que l'union d'une partie de ce dernier avec le premier est constante. Je ne l'ai pas trouvée sur un exemplaire, mais il s'agissait probablement d'une détérioration dûe à la recherche des autres nerfs. Différentes variations peuvent se présenter, notamment le premier nerf spinal ne vient pas toujours s'appliquer contre le bord dorsal du nerf occipital, il peut même en rester assez éloigné; sa division en deux filets est alors plus nette et le filet antérieur qui se fusionne au nerf occipital est plus long. L'état décrit plus haut était celui d'un exemplaire disséqué spécialement pour la préparation du nerf occipital et des premiers nerfs spinaux. Il est donc certain qu'au moment où le nerf descend devant la barre osseuse du supraclaviculaire, c'est un complexe formé des éléments ventraux et des éléments dorsaux de ses deux racines et d'un faible contingent sensible ou moteur, ou peut-être senso-moteur, provenant du premier nerf spinal. En tout cas, une bonne partie des éléments sensibles ne prend pas part au trajet ventral du nerf; elle se détache pour constituer le rameau peaucier destiné au coude de l'os claviculaire.

Le complexe du nerf occipital et du premier nerf spinal, débarrassé d'une forte fraction de ses fibres sensibles, se bifurque comme nous l'avons vu. Sa partie postérieure prend part à la 
formation du plexus brachial; sa partie antérieure devient le nerf de la région située entre l'extrémité inférieure de la clavicule et l'os hyoïde. Pour cornaître exactement la provenance des éléments de ce nerf, il faudrait savoir d'où proviennent ceux du rameau peaucier de la clavicule et comment le reste des éléments du complexe se répartit au moment de sa bifurcation. Cette étude n'étant pas faite, on doit s'abstenir de conclusions précises. On peut cependant affirmer que la branche ventrale du complexe est constituée en majeure partie de fibres motrices fournies par la racine ventrale du nerf occipital. Elle peut recevoir aussi des éléments moteurs du premier nerf spinal; ceux-là ne pourraient cependant représenter qu'une fraction très faible de la partie motrice. En outre, la branche ventrale est formée d'une partie sensible peu importante, comprenant quelques filets destinés à la peau qui recouvre la face inférieure du muscle sterno-hyoïdien; cette partie sensible peut également dépendre du nerf occipital, du nerf spinal ou des deux à la fois.

D'après nos observations sur le Silure, les relations du nerf occipital ne seraient pas si simples que Büchner et Stannius l'ont pensé. En effet, le nerf occipital ne se divise pas simplement en une partie antérieure qui devient la branche ventrale et une partie postérieure qui s'unit au premier nerf spinal, mais c'est le premier nerf spinal qui envoie un rameau de renforcement au nerf occipital et c'est seulement le tronc produit par cette union qui se bifurque en une branche ventrale et en un tronçon pour le plexus branchial. Il ne s'agit pas non plus du fusionnement du premier spinal avec le nerf occipital, comme FürbRinger l'a trouvé et représenté chez le Brochet (99, Pl. 8, fig. 5), mais d'un état ressemblant à celui que le même auteur a rencontré chez Caranx trachurus (Pl. 8, fig. 7).

Les nerf's qui naissent postérieurement au nerf occipital son 
des nerf spinaux. Je dirai quelques mots des premiers, pour autant que ces renseignements peuvent faire comprendre ce qui a été exposé précédemment, car ils présentent des modifications nombreuses causées, en partie par le voisinage du membre antérieur, en partie par la présence de l'appareil de WEBER.

Jusqu'au niveau de la première apophyse épineuse indépendante (Pl. 1, fig. 1, A $)$, c'est-à-dire non soudée à l'apophyse épineuse d'une autre vertèbre ou avec le crâne, on compte quatre nerfs spinaux. Le quatrième passe devant l'arc supérieur qui porte cette première apophyse épineuse indépendante. Il sort par une racine ventrale et une racine dorsale qui longent la moelle épinière d'avant en arrière sur un petit trajet, et quittent le canal neural par deux trous distincts. Les deux racines se rejoignent à l'extrémité distale du ganglion formé par la racine dorsale. Le ganglion est logé dans l'angle antérieur situé entre la paroi du canal rachidien et cette forte pièce osseuse à deux prolongements qui doit représenter l'arc inférieur (apophyse transverse) de la deuxième vertèbre (fig. 2, $\mathrm{Ai}_{2}$ ). Du ganglion, part le rameau dorsal ( $\mathrm{Pl}$. 3, fig. 3, IV d), qui s'élève entre les deux masses dorsales du muscle latéral en s'inclinant légèrement en arrière et se divise en deux à quatre filets qui entrent dans le nerf de WEBER. La branche ventrale (fig. 3,4 $\mathrm{IVv}_{\mathrm{v}}$, très forte par rapport aux branches ventrales des nerfs spinaux postérieurs, s'infléchit latéralement et en arrière et passe sous la forte apophyse du deuxième arc inférieur qui sert d'appui à la ceinture scapulaire; elle réapparaît dans l'échancrure située entre la pointe postérieure et l'apophyse du deuxième arc inférieur, descend entre le muscle ventro-latéral et la peau et atteint la nageoire pectorale vers la pièce basale postérieure. Le quatrième nerf spinal est le dernier qui prenne part à l'innervation du membre antérieur; de plus, il ne contribue pas à la formation du plexus brachial, ses relations avec les nerfs pré- 
cédents n'ayant lieu qu'entre des rameaux tout à fait périphériques.

La distance entre le quatrième et le troisième nerf spinal est presque normale, elle est seulement un peu plus faible que la distance entre les nerfs postérieurs. Par contre, la distance entre le troisième et le deuxième, et entre le deuxième et le premier, est beaucoup moins grande; elle est réduite environ de moitié.

Le troisième nerf est le plus volumineux des quatre. Ses racines se comportent, dans le canal neural, comme celles du quatrième, mais elles ont ceci de particulier qu'elles sortent en même temps que les racines du deuxième nerf spinal, par un trou assez grand recouvert d'une membrane résistante. Ce trou est limité en avant par l'un des osselets de l'appareil de WEBER, le stapes (fig. $2, \mathrm{~S}$; arc inférieur de la $1^{\text {re }}$ vertèbre) et il est beaucoup plus grand que ne le demande le passage des racines. Il s'agit probablement ici d'une modification due à la présence de l'appareil de WeBer, la partie membraneuse de la paroi du canal neural facilitant évidemment beaucoup les mouvements du stapes. Enfin, l'arc vertébral percé de ce trou doit être regardé comme produit par le fusionnement des arcs supérieurs des deuxième et troisième vertèbres, dont les corps sont également soudés sans qu'il reste de traces de leurs limites.

Le troisième nerf spinal a également son ganglion au point de réunion des deux racines, soit immédiatement à la sortie du canal neural; pour y parvenir, celles-ci s'infléchissent un peu en avant. Du ganglion part un fort rameau dorsal (fig. 3, III d) qui s'élève dans l'interstice médian en s'inclinant un peu en avant, de telle façon qu'il passe d'abord au-dessus de la racine dorsale du deuxième nerf spinal; il se divise et se rend dans le nerf de WEBER, comme le quatrième, mais auparavant, sa plus forte partie se réunit généralement à une partie du rameau dorsal du deuxième nerf spinal. La branche ventrale, la plus 
forte de toutes, passe au-dessus du malleus (fig. 2, M; arc inférieur de la $3^{\text {me }}$ vertèbre) et reçoit un peu plus loin un fort faisceau venant du deuxième nerf spinal. Ensuite, elle longe inférieurement l'apophyse scapulaire du deuxième arc inférieur, arrive contre la face interne de la clavicule et s'infléchit en arrière en passant contre l'origine du fort muscle adducteur supérieur de la nageoire; elle pénètre donc dans la musculature de la face postérieure de la pectorale.

Les deux racines du deuxième nerf spinal ont aussi un court trajet d'avant en arrière contre la moelle épinière. Elles se replient vers l'avant, une fois sorties du canal, et se réunissent au point où la racine dorsale forme son renflement. Le rameau dorsail (fig. 3, II d) est également fort, incliné un peu vers l'avant et croise aussi, au départ, la racine dorsale du nerf précédent. La branche ventrale (II v) très forte encore, quoique un peu moins que celle du troisième nerf, passe au-dessus du malleus et se bifurque. Le faisceau posterieur s'unit à la branche ventrale du troisième nerf. Le faisceau antérieur descend derrière la barre osseuse du supra-claviculaire, s'unit avec un rameau du premier nerf spinal, puis parvient contre la face médiale de la clavicule où il reçoit encore un faisceau venant du complexe formé par le nerf occipital et le premier nerf spinal. Le tronc ainsi constitué envoie des rameaux à la musculature de la face antérieure de la nageoire pectorale et se prolonge dans la partie du muscle ventro-latéral insérée sur la face inférieure de la clavicule.

Enfin, le premier nerf spinal est très faible. La racine ventrale est grêle et la racine dorsale l'est encore davantage; elles ont aussi un court trajet d'avant en arrière, le long de la moelle, sont immédiatement accolées l'une contre l'autre et sortent par une petite fente entre le stapes (S) et le claustrum (Clt). Le filet ainsi formé n'atteint pas $1 \mathrm{~mm}$. de diamètre. En sortant du canal rachidien, il n'est séparé du deuxième nerf spinal que 
par l'étroite branche montante du stapes; il s'infléchit en avant et latéralement en passant au-dessous du deuxième rameau dorsal et ne forme un petit renflement qu'après s'être beaucoup rapproché du nerf occipital. De ce ganglion part un faible rameau dorsal (I d) qui s'élève contre l'occipital latéral et l'occipital supérieur et ne s'unit pas au nerf de WEBER comme les rameaux dorsaux des nerfs suivants; il passe contre la crête du supra-occipital, un peu en avant de l'orifice de sortie du nerf de Weber, et se rend dans la peau, après s'être anastomosé a vec des filets sortant du nerf de WEBER. La branche ventrale va latéralement et un peu en avant, descend devant la barre osseuse du supra-claviculaire, se divise, comme je l'ai déjà expliqué, en un court faisceau antérieur qui se joint au nerf occipital et en un faisceau postérieur qui s'unit à la branche ventrale du deuxième nerf spinal.

L'espace situé entre les racines du premier nerf spinal et celles du nerf occipital est toujours beaucoup plus considérable que les intervalles séparant les quatre premiers nerfs spinaux ; elle atteint et dépasse $1,5 \mathrm{~cm}$. Entre deux, il n'y a aucune trace du nerf qui devrait sortir entre le bord de l'occipital latéral et le claustrum et qui correspondrait au troisième nerf occipital décrit par SAgemenl chez Amia. On sait que, d'après cet auteur, le claustrum est probablement un arc occipital resté libre, fonctionnant comme osselet de l'appareil de WEBER, et que c'est à la mobilité de cet osselet contre le bord de l'occipital latéral qu'il faudrait attribuer la disparition du nerf correspondant.

\section{RÉsuné et Conclusions}

\section{A. Musculature.}

Chez le Silure, les six muscles oculaires sont présents, les quatre droits sont très allongés et minces, les obliques relativement courts. 
L'adducteur mandibulaire comprend une portion superficielle et une portion profonde dont les ventres sont parfaitement distincts. Chacune de ces portions s'insère en partie sur l'articulaire, mais elles ont un prolongement tendineux commun qui redevient musculeux en avant et s'insère sur le cartilage de Meckel et sur la face interne du dental. C'est un stade moins différencié que celui de la plupart des autres Téléostéens et en particulier du Brochet.

La musculature du barbillon maxillaire se compose de deux extenseurs et d'un rétracteur. D'après leur situation et leur innervation, les deux extenseurs semblent provenir de la musculature de l'arc hyoüdien et appartenir au domaine du facial, tandis que le rétracteur dérive de la musculature de l'arc maxillaire et appartient au domaine du trijumeau.

La musculature dorsale des arcs maxillaire et hyoïdien présente les particularités suivantes : l'élévateur palatin est, en grande partie, intercalé entre les deux portions de l'adducteur mandibulaire; d'autre part, sa parenté avec le dilatateur operculaire est rendue très évidente: $1^{0}$ par la présence chez ce dernier d'une longue portion antérieure contiguë à l'origine de l'élévateur palatin et d'une portion postérieure (la seule qui existe chez les autres Téléostéens); $2^{\circ}$ par l'innervation des deux muscles par un même rameau du tronc maxillaire commun. L'adducteur palatin et les deux extenseurs du barbillon sont innervés par un même rameau du tronc hyoïdeo-mandibulaire; l'adducteur hyomandibulaire, l'élévateur et l'adducteur operculaires par un autre rameau de ce tronc.

La musculature dorsale des arcs branchiaux se compose :

$1^{\circ}$ De quatre élévateurs offrant un état moins élevé que ceux des autres Téléostéens, car ils ne correspondent qu'aux élévateurs externes de ceux-ci; de plus, les deux postérieurs, destinés aux acs III et IV, montrent dans leur origine et leur insertion une disposition tout à fait spéciale, qui doit résulter de 
l'adaptation de ces deux muscles comme élévateurs de la plaque pharyngienne dentée.

$2^{\circ}$ D'obliques dorsaux situés seulement sur la face supérieure des extrémités dorsales des arcs. Ils ne sont pas divisés en muscles distincts pour chaque arc et leurs fibres postérieures se prolongent, en partie, dans le constricteur pharyngien.

$3^{\circ}$ De deux parties du constricteur pharyngien qui tendent à se spécialiser comme muscle de l'appareil branchial. L'une relie le coude du quatrième arc à l'extrémité voisine de l'os pharyngien inférieur, l'autre, l'épibranchial du quatrième arc au cérato-branchial correspondant.

$4^{\circ}$ Des transverses dorsaux représentés par une plaque musculaire non divisée, étendue d'un côté à l'autre à la face inférieure des trois premiers arcs, et touchant en arrière au constricteur pharyngien. L'innervation par les deuxième et troisième troncs branchiaux montre que cette plaque est bien l'homologue d'au moins deux des traverses dorsaux d'autres Poissons.

5. D'un rétracteur antérieur et d'un rétracteur postérieur, non comparables aux rétracteurs connus chez d'autres Téléostéens. Leur situation et leur innervation indiquent qu'ils proviennent probablement d'obliques dorsaux modifiés.

La musculature ventrale des ares maxillaire et hyoïdien comprend :

$1^{\circ}$ Un muscle intermandibulaire représenté par la partie antérieure du génio-hyoïdien dont il n'est rendu distinct que par une étroite inscription tendineuse.

$2^{\circ}$ Un muscle génio-hyoïdien appartenant exclusivement au domaine du trijumeau.

$3^{\circ}$ Deux hyo-hyoïdiens dont l'inférieur n'est pas un simple prolongement du supérieur, mais possède une origine très étendue sur l'arc hyoïdien. Les deux muscles ne sont pas complètement indépendants l'un de l'autre; ils ont un prolongement tendineux commun inséré sur l'hypohyal. Chez les autres Téléostéens, une 
partie du hyo-hyoïdien inférieur doit s'adjoindre au génio-hyoïdien, ce qui explique en même temps pourquoi ces Téléostéens ont une région postérieure du génio-hyoüdien innervée par le nerf hyoïdien et pourquoi le hyo-hyoïdien du Silure a un si grand développement.

La musculature ventrale des arcs branchiaux comprend:

$1^{\circ}$ Un pharyngo-arcuo-hyoïdien, masse musculaire commune à l'arc pharyngien incomplet, aux arcs branchiaux et à l'arc hyoïdien. De ce muscle, on peut faire dériver les obliques ventraux présents sous des états variables chez la plupart des Poissons osseux, le pharyngo-hyoïdien que l'on ne connaît encore que chez la Perche et le pharyngo-arcual, connu sous des formes différentes chez le Brochet et les Cyprins.

$2^{\circ}$ et $3^{\circ}$ Un transverse ventral et un pharyngien transverse.

$4^{\circ}$ Deux pharyngo-claviculaires appartenant au domaine du vague et non à celui du "rameau hypoglosse » dont ils dépendent chez d'autres Poissons, d'après des ouvrages récents.

La musculature longitudinale ventrale est représentée seulement par le sterno-hyoüdien, naissant presque exclusivement sur la face interne de la clavicule.

La musculature céphalo-scapulaire ne possède pas de muscle trapézoïde différencié. Le fort prolongement du muscle dorsolatéral qui en tient lieu s'avance jusque sur l'os frontal.

\section{B. Nerfs.}

L'olfactif appartient au type primitif, dans lequel le bulbe olfactif, situé à l'entrée de la fosse nasale, est relié au prosencéphale par un long tractus logé dans la cavité cranienne.

L'optique est allongé et mince; vu l'absence de cavité orbitaire proprement dite, il passe entre les muscles de la mandibule et de la voûte palatine.

Les nerfs moteurs oculaires sont indépendants de l'ophtalmi- 
que, mais le trochléaire se joint à l'oculo-moteur commun dans la cavité cranienne et en sort avec lui, de telle manière que le nerf destiné au muscle oblique supérieur semble être un rameau de l'oculo-moteur commun. L'abducteur est complètement indépendant. Le trochléaire et l'abducteur décrits par Stannius comme sortant de l'ophtalmique ne peuvent être que des ramifications de celui-ci, destinées au globe de l'œil et au tégument environnant. Pas de ganglion ciliaire visible. Les trois nerfs ont chacun leur origine distincte sur la moelle allongée comme généralement.

Les nerfs trijumeau et tacial forment un complexe dans le tronc duquel on peut reconnaître trois composants senso-moteurs, mais que l'on ne peut pas séparer complètement à cause des passages d'éléments de l'un à l'autre.

L'existence de ces composants ne peut être expliquée qu'en admettant la formation du trijumeau facial par trois nerfs segmentaux. Le composant postérieur entre en grande partie dans le tronc hyö̈leo-mandibulaire et dans le nerf de WEBER, le composant antérieur forme la région supérieure des deux nerfs maxillaires et une partie de l'ophtalmique, le composant moyen constitue la région inférieure des deux nerfs maxillaires et une partie de l'ophtalmique.

Branches du trijumeau-facial :

$1^{\circ}$ Il y a deux branches ophtalmiques. La branche superficielle comprend deux rameaux, un supérieur et un inférieur, qui se distribuent à la peau et aux canaux muqueux des régions frontale, ethmoïde et prémaxillaire; de plus, le rameau inférieur émet un rameau oculo-nasal comprenant un nerf ciliaire et d'autres ramifications destinées aux orifices nasaux, aux environs de l'œil et aux canaux muqueux des os sous-orbitaires. Les deux rameaux de la branche ophtalmique superficielle correspondent aux ophtalmiques supérieur et inférieur de Stannius. La branche ophtalmique profonde se distribue dans la peau autour 
de la fosse nasale, à l'orifice nasal antérieur, dans la région prémaxillaire et à la base du barbillon. La grande extension du nerf ophtalmique du Silure et sa division en nombreuses ramifications correspondent simplement à la grande largeur que les pièces squelettiques médianes de la tête acquièrent chez ce Poisson.

$2^{\circ}$ Les branches maxillaires supérieure et inférieure sortent d'un tronc commun court. Ce dernier et la base des deux branches sont divisés en deux parties superposées, alimentées principalement par les composants antérieur et moyen du complexe. La situation et les connexions de ces composants permettent d'admettre que le maxillaire supérieur est surtout formé d'éléments du trijumeau antérieur et du trijumeau postérieur, le maxillaire inférieur du trijumeau antérieur et du facial. Le tronc commun émet les rameaux suivants avant de se bifurquer : un rameau de la muqueuse buccale, un rameau de l'élévateur palatin et du dilatateur operculaire, un rameau de l'adducteur mandibulaire et du rétracteur du barbillon, et enfin le rameau sphéno-palatin qui paraît être sans relation avec le tronc hyoïdeo-mandibulaire.

La branche maxillaire supérieure devient le nerf sensible du barbillon; elle émet un seul rameau qui se fusionne temporairement avec l'ophtalmique profond et constitue un rameau alvéolaire prémaxillaire anastomosé avec le sphéno-palatin.

La branche maxillaire inférieure fournit le nerf postérieur du barbillon maxillaire et se divise en deux branches. La branche mandibulaire externe, très forte par rapport à celle des autres Téléostéens, se termine par un rameau alvéolaire et un rameau labial. La branche mandibulaire interne ne se fusionne nullement avec la branche mandibulaire interne du tronc hyoïdeomandibulaire; elle innerve donc seule les muscles intermandibulaire et génio-hyoïdien; en outre, elle émet un rameau alvéolaire, les nerfs sensibles des deux barbillons mandibulaires et de nombreux rameaux destinés à la région environnante. 
$3^{\circ}$ La branche hyoïdeo-mandibulaire est formée principalement par le facial, mais elle reçoit aussi des éléments du trijumeau postérieur. Il existe un rameau communicant venant du tronc que forme le reste du complexe trijumeau-facial, mais il est très court. La branche fournit un rameau destiné à l'adducteur palatin et aux deux extenseurs du barbillon, un autre rameau innervant l'adducteur hyomandibulaire, l'élévateur et l'adducteur operculaires, puis elle se divise en un nerf mandibulaire et un nerf hyoïdien.

Le nerf mandibulaire comprend deux branches. L'interne correspond à l'unique branche des autres Téléostéens; l'externe destinée complètement à la peau de la mandibule acquiert, comme la branche externe issue du maxillaire inférieur, une très grande extension. Une innervation aussi riche du tégument n'est qu'une compensation de l'insuffisance visuelle. Les deux branches mandibulaires interne et externe ne sont pas homologues aux ramifications appelées quelquefois nerfs mandibulaire et dentaire.

Le nerf hyoïdien innerve les deux hyo-hyoïdiens et donne des rameaux peauciers à la membrane branchiostège; il reste complètement étranger à l'innervation du génio-hyoïdien.

$4^{\circ}$ Le nerf de WEBER, ou branche dorsale du trijumeau-facial, tire son origine des trois composants du plexus; il peut donc être regardé comme le rameau dorsal du trijumeau antérieur, du trijumeau postérieur et du facial. Il n'est pas en communication avec le nerf latéral du vague et son trajet n'est pas superficiel. Il est le collecteur des rameaux dorsaux de tous les nerfs spinaux et envoie des ramifications peaucières à toute la région dorsale.

Le nerf acoustique comprend deux rubans, contigus à la sortie de la moelle allongée; le premier innerve les ampoules des canaux antérieur et externe et l'utricule, le second le saccule et l'ampoule du canal postérieur. L'acoustique ne reçoit pas de faisceau du facial. En outre, il n'existe pas d'auditif accessoire

Rev. Suisse de Zool., T. 6. 1898. 
(WEBER); c'est probablement le cordon postérieur que l'on avait à tort désigné ainsi.

Le nerf glosso-pharyngien sort de la moelle allongée par deux composants distincts de ceux du vague, mais renforcés par un faisceau provenant de ce dernier nerf. Le ganglion se forme à la sortie du crâne; la partie motrice du nerf se sépare alors de la partie sensible et s'y réunit ensuite. Le nerf envoie des rameaux au rétracteur branchial antérieur et à l'élévateur du premier arc, puis devient le nerf antérieur de cet arc; ses rameaux terminaux vont dans la partie antérieure du pharyngoarcuo-hyoïdien et dans la région avoisinante de la muqueuse buccale. Une branche antérieure longeant l'arc hyoïdien manque complètement.

Le nerf vague naît par deux composants, dont l'antérieur, compact, représente une partie de la racine dorsale et devient le nerf latéral, et dont le postérieur, formé de trois faisceaux très rapprochés, représente la racine ventrale plus une partie de la racine dorsale et devient le vague proprement dit. Le composant postérieur émet un faible rameau dorsal qui se perd dans la cavité cranienne et il reçoit du composant antérieur un faisceau de renforcement.

Le vague proprement dit comprend :

$1^{\circ}$ Trois troncs branchiaux munis chacun d'un ganglion à leur base et répartis dans les arcs selon le schéma général. Ils innervent les obliques dorsaux, le transverse dorsal ( $2^{\text {e }}$ tronc), tous les élévateurs branchiaux sauf le premier, le rétracteur postérieur ( $3^{\mathrm{e}}$ tronc), le constricteur du quatrième arc ( $3^{\mathrm{e}}$ tronc), le pharyngo-arcuo-hyoïdien et le transverse ventral.

$2^{\circ}$ La branche pharyngienne inférieure envoie des rameaux à la région dorsale du constricteur pharyngien, d'autres à la région latérale et ventrale du même muscle, à la muqueuse de l'os pharyngien inférieur et du pharynx. La branche pharyngienne inférieure peut être comparée à un tronc branchial dont 
la branche antérieure devient le nerf postérieur du quatrième arc et dont la branche postérieure a subi des modifications secondaires. L'arc pharyngien ayant perdu sa fonction respiratoire et acquis un rôle actif dans l'acte de la déglutition, les éléments sensibles et vaso-moteurs de la branche ont été soumis à une réduction; au contraire, les éléments moteurs ont acquis un développement proportionnel à celui de la musculature spéciale de l'arc (transverse pharyngien, pharyngo-claviculaires, et probablement partie postérieure du pharyngo-arcuo-hyoïdien).

$3^{\circ}$ La branche pharyngo-cardiaque envoie à la région dorsale du pharynx des filets qui ne lui sont probablement adjoints que temporairement. Le rameau cardiaque va dans l'oreillette.

$4^{\circ}$ La branche intestinale court le long de l'œsophage et de l'estomac et livre de nombreuses ramifications à la musculature et à la muqueuse.

Le nerf latéral du vague, formé exclusivement d'éléments sensibles, ne livre à la tête qu'un rameau operculaire entrant en connexion momentanée avec le rameau operculaire du tronc hyoïdeo-mandibulaire, et un rameau supra-claviculaire. Dans le tronc, il est situé entre les masses dorsale et ventrale du muscle latéral. En avant, il émet une branche superficielle placée bien au-dessous de la ligne latérale; elle innerve seulement les deux premiers pores de cette dernière et livre un rameau ventral au niveau de chaque myomère du tronc. Dans chaque segment du tronc (à part les deux premiers) et de la région caudale, le nerf latéral envoie un filet qui s'avance dans l'interstice intermusculaire, accompagné du rameau intermédiaire du nerf spinal correspondant, se ramifie à la périphérie et innerve le canal latéral (possédant un pore par myomère), ainsi que la peau située audessus. Il y a de fréquentes anastomoses, à la périphérie, entre les filets du nerf latéral et ceux des rameaux intermédiaires, mais il n'y a pas renforcement du nerf latéral par des éléments des nerfs spinaux. 
La branche superficielle du nerf latéral, chez le Silure, ne doit pas être comparée à celle de la plupart des Téléostéens qui se forme par juxtaposition des extrémités périphériques de tous les rameaux de l'interstice. Elle ne peut, au contraire, avoir été produite que par le déplacement vers l'avant du point où les deux premiers rameaux se séparent du nerf. Il se forme ainsi une branche qui fournit alors les rameaux des deux premiers myomères et acquiert un grand développement dans la région ventrale des autres myomères du tronc, domaine qui lui est primitivement étranger.

Le système latéral du Silure n'offre donc pas un état anormal comme d'anciens auteurs l'ont pensé, mais peut être parfaitement rattaché au plan général. Enfin, le Silure montre que le nerf latéral peut être très volumineux, même lorsque le canal latéral est très peu développé. C'est un nerf de sensibilité générale, en relation avec les organes sensoriels de la ligne latérale; lorsque la peau est dépourvue d'écailles, l'utilité du canal latéral diminue, celui-ci est soumis à une réduction, mais les ramifications du nerf latéral forment un réseau peaucier plus riche et le nerf conserve toute son importance.

Le nerf occipital a bien conservé les caractères d'un nerf spinal. Il naît par deux racines distinctes, dont la dorsale a un ganglion situé dans la cavité cranienne, mais ne donne pas de rameau dorsal. Le nerf sort, en arrière du vague, par un orifice particulier de l'occipital latéral. Il reçoit un court rameau communicant de la branche ventrale du premier nerf spinal (très faible), envoie un rameau peaucier au coude de la cla. vicule et se bifurque. La partie postérieure s'unit avec une partie du deuxième nerf spinal, déjâ renforcée par le premier nerf spinal; la partie antérieure se rend dans le sterno-hyoüdien et dans le revêtement cutané. Il n'y a donc pas d'hypoglosse proprement dit, mais un complexe formé du nerf occipital et d'une partie du premier nerf spinal et dont la branche antérieure 
innerve la région située entre les extrémités ventrales de la clavicule et de l'arc hyoïdien, laquelle comprend le domaine de l'hypoglosse.

Enfin, on rencontre chez le Silure un grand nombre de variations individuelles et de cas d'asymétrie, par exemple dans les ramifications de l'ophtalmique, dans le rameau adducteur mandibulaire, dans les rameaux ascendants du trijumeau-facial, dans les rameaux dorsaux des nerfs spinaux se jetant dans le nerf de WEBER, dans la branche superficielle du nerf latéral, et dans la distance entre le nerf vague et le nerf occipital. Ces variations ont une grande importance, parce que, le plus souvent, elles accompagnent des modifications en vue d'adaptations spéciales et en sont ou des formes de passage, ou des stades extrêmes.

\section{ADDENDA}

Le mémoire de M. JAQUET : Recherches sur l'anatomie et l'histologie du Silurus glanis (avec 13 pl. Arch. des Sciences médicales de Bucarest, $\mathrm{n}^{\text {os }} 3$ et 4 , mai et juillet, Paris 1898) m'est parvenu alors que l'impression de mon travail était ache. vée. Bien que cet ouvrage ne comporte ni l'étude de la musculature, ni celle des nerfs, je regrette de n'avoir pas pu tenir compte de ses indications, qui diffèrent des miennes au sujet de l'interprétation de quelques pièces squelettiques. La comparaison des figures suffira, du reste, à éclairer le lecteur. 


\section{INDEX BIBLIOGRAPHIQUE.}

Les ouvrages que l'auteur n'a pas pu consulter sont marqués d'un astérisque.

1. - Gall et Spurzherm. Anatomie et physiologie du systeme nerveux en général et du cerveau en particulier. 4 vol. et atlas. Paris, 1810-1819.

2. - E.-H. Weber. De aure et auditu hominis et animalium. av. pl. Leipzig, 1820 .

3. - Desmoulins. Recherchos anatomiques et physiologiques sur le systeme nerveux des poissons. Magendie, Journal de Physiologie. Tome 2, 1822, p. 127-135 et p. 348-353. - En allemand, dans : Meckel's deutsch. Archiv. f. Physiol. Bd. 7. 1822 , p. 566-571 ; Bd. 8. 1823, p. 185-190.

4. - Flourens. Recherches expérimentales sur les propriétés et les fonctions du système nerveux dans les animaux vertébrés. Paris, 1824.

5. - Desmoulins et Magendie. Anatomie des systemes nerveu $x$ des animaux a vertebres. 1 vol. et atlas. Paris, 1825.

6*. - E.-H. WeBer. Ueber vier Längennerven bei einigen Fischen, von denen zwei von dem Trigeminus und zwei von dem Vagus entspringen, die die ganze Länge der Rumpfes durchlaufen. Mit Abbildgn. Meckel's Arch. f. Anat. u. Phys. 1827, p. 303-308.

7. - G. Cuvier et A. Valenciennes. Histoire naturelle des Poissons. Vol. 1-18 et cahiers de pl. 1-25, in- $8^{\circ}$. Paris, 1828-45.

8. -- Bischoff. Nervi accessorii Willisii anatomia et physiologia. Heidelberg, 1832 .

9. - G. Büchner. Memoire sur le systeme nerveux du barbeau Cyprinus barbus L., av. 1 pl. Mém. de la Soc. d'hist. nat. de Strasbourg. T. II, 1835. 57 p. - Extrait dans l'Institut. T. IV, 1836, No 174, p. 296-298.

10. - Schlemm und E. D’Alton. Ueber das Nervensystem der Petromyson. Müller's Archiv. f. Anat. 1838, p. 262-273. Erklärung der hierzu gehörigen Kupfertafeln, ibid. 1840 , p. 5-14.

11. - Leuret et Gratiolet. Anatomie comparée du système nerveux. Paris, 1839-1857. 2 vol. $8^{\circ}$, atlas fo.

12. - Longet. Anatomie et Physiologie du systeme nerveux. 2 vol., 8 pl. Paris, 1842.

13. - G. Cuvier. Leçons d'anatomie comparée. Paris, 1845. T. I-III.

14. - A. Prevost. Recherches sur le système nerveux de la téte du Congre (Conger vulgaris). 1 pl. Mém. de la Soc. de Phys. et d'Hist. nat. de Genève. T. 11. 1846, p. 191-224. - A part, Genève. 1846, $4^{\circ}$.

15. - Girgensohn. Anatomie und Physiologie des Fisch-Nervensystems. Mit 15 Taf. Mém. près. Acad. St-Pétersbourg. T. 5. 1846, p. 257-589.

16. - H. Stannius. Das peripherische Nervensystem der Fische, anat. u. physiol. untersucht. Mit 5 Steintafeln. Rostock, Stiller, 1849. 156 p.

17. - Leydig. Ueber die Schleimkanäle der Knochenfische. Mit Taf. Müller's Archiv. 1850, p. 170-181.

18. - Frantzius. Beiträge zur Entwicklungsgeschichte des peripherischen Nervensystems. Zeitschr. f. wiss. Zool. Bd. 3. 1851, p. 510-521. 
19*. - H. Stannius. Zootomie der Fische. Berlin, 1854.

20. - C. Vogt. Ueber die Schleimkanäle der Fische. Zeitschr. f. wiss. Zool• Bd. VII, 1856. p. 328-329.

21. - Carl-Ernst-Emil Hoffmann. Beiträge zur Anatomie und Physiologie des Nervus vagus bei Fischen. Nebst 1 (lith.) Taf. Abbildg. Pr venia legendi Med. Facult. Giessen. Giessen, Druck von Wilh. Keller, 1860. 4 ${ }^{\circ}, 31$ S. Auch imHandel: Giessen, Ricker, 1860. $4^{\circ}$.

22. - A. Moreau. Recherches anatomiques et physiologiques sur les nerfs du sentiment et du mouvement chez les poissons. Ann. Scienc. nat. 4. Sér. Zool. T. 13,1860 , p. 380-382.

23. - L. Beale. Observations générales sur la distribution périphérique des nerfs. (Trad.) Journal de la Physiol. (Brown-Séquard). T. 5. 1862. p. 288-292.

24. - A. Günther. Catalogue of Fishes in the British Museum. Vol. V. 1864. 25. - Ch. Vouga. Sur les qualités électriques chez notre Silure (Silurus glanis). Actes de la Soc. helvét. des Sc. nat. Neụchàtel, 50e session, 1866. p. 98-99.

26. - E. BAUdelot. Considerations sur quelques particularités du systeme musculaire chez les Poissons. Paris, Compt. rend. LXIV, 1867, p. 1205-1208.

27. - E. Baudelot. De la détermination des pièces osseuses qui se trouvent en rapport avec les premières vertëbres chez les Cyprins, les Loches et les Silures. Paris, Compt. rend. Ac. sc. LXVI, 1868, p. 330-334.

28. - L. Stieda. Studien über das centrale Nervensystem der Knochenfische. Zeitschr. f. wiss. Zool. Bd. 18. 1868.

29. - F. FÉE. Recherches sur le système latéral du nerf pneumo-gastrique des poissons. Av. 4 pl. Mém. Soc. Sc. nat. Strassbourg. T. 6, 2e livr. 1870. p. 129-201.

30. - Арамӥк. Ueber die Innervation der Augenbewegungen. Medic. Centralbl. 8. Jahrg. 1870, p. 65-67. - Extrait en français dans Arch. sc. phys. et nat. Genève. Nouv. pér. T. 38, 1870, p. 299-304.

31. - Gegenbaur. Grundzüge der vergleichenden Anatomie. Leipzig, 1870.

Manuel d'anatomie comparée Trad. de C. Vogt, Paris 1874.

32. - Gegenbaur. Ueber die Kopfnerven von Hexanchus und ihr Verhältniss zur « Wirbeltheorie» des Schädeis. Mit 1 Taf. - Jena. Zeitschr. 6. Bd. 1871, p. $497-559$.

33.. - Humphry. Muscles a Nerves of Lepidosiren. Journ. of Anat. and Physiol. 2e sér. Vol. V. 1871-1872.

34. - Humphry. Muscles of Ceratodus. Journ. of Anat. a. Physiol. Vol. VI, 1872 , p. 279-287.

35. - Gegenbaur. Untersuchungen zur vergleichenden Anatomie der Wirbelthiere. Leipzig, 1872. III. Heft.

36. - Jobert. Etudes d'anatomie comparie sur les organes du toucher chez divers Mammifères, Oiseaux, Poissons et Insectes. Av. pl. Ann. sc. nat., 5 série, T. XVI, 1872, 162 pages.

37. - B. Vetter. Untersuchungen zur vergl. Anat. der Kiemen und Kiefermuskulatur der Fische. Jen. Zeitschr. 1. Theil. Bd. VIII (N. F.), 1874.

38. - E. Baudelot. (†1875). Recherches sur le systeme nerveux des poissons, av. $10 \mathrm{pl}$. Paris, Masson, 1883. Fol. 178 pages.

39. - C. Bruch. Vergleichende Osteologie des Rheinlachses. 2. Aufl. Mainz, 1875 . 
40. - P. Fürbringer. Muskulatur und Nervensystem der Cyclostomen. Jen. Zeitschr. Bd IX. 1875, p. 1-93, 3 pl.

41.. - JACKson and CLARKE. The Brain and Cranial Nerves of Echynorhinus spinosus with notes on the other viscera. With 1 Pl. Journ. of Anat. a. Physiol. Vol. 10. 1876, p. 75-107.

42. - Вакошіескі. Zur Frage vom Verwachsen der peripherischen Nerven. Vorläufige Mittheilung. Mit 1 Taf. Archiv. f. mikr. Anat. 13. Bd. 1877, p 420-426.

$43^{\circ}$. - E. Baudelot. Note sur un procédé relatif à la dissection du système nerveux chez les poissons. Rev. Sc. nat. T. 6, 1877, p. 237-240.

44. - J. V. Rohon.-Das Centralorgan des Nervensystems der Selachier. Mit 9 Taf. Wien, 1877 .

45. - Bridge. The Cranial Osteology of Amia calva. With 1 Pl. Journ. of Anat. a. Physiology. Vol. XI, 1878, p. 605-622.

46. - C. Gegenbaur. Ueber das Kopfskelet von Alepocephalus rostratus Risso, nebst Bemerkungen über das "Kiemenorgan» von Alausa vulg. C. V. Mit 2 Taf. u. 1 Holzschn. Morphol. Jahrbuch, 1878, 4 Bd. suppl. p. 1-42.

47. - J. V. Rohon. Ueber den Ursprung des Nervus vagus bei Selachiern mit Berücksichtigung der Lobi electrici von Torpedo. 1 Taf. Wien, 1878.

48. - B. Vetter. Untersuchungen zur vergleichenden Anatomie der Kiemen und Kiefermuskulatur der Fische. 2. Theil. Jen. Ztschr. Bd. XII, 1878, 3. Heft, p. 431.550 .

49. - A. Friant. Recherches anatomiques sur les nerfs trijumeau et facial des poissons osseux. Av. 6 pl. Bull. Soc. Sc. Nancy. 2 Sér. T. IV, Fasc. IX, 1879, p. 1-108. - Aussi séparément: Nancy, Berger-Levrault et Ce, 1879, roy. $8^{\circ}$.

50. - M. Fürbringer. Zur Lehre von den Umbildungen des Nervenplexus. Morphol. Jahrb. Bd. V, 1879, p. 324-394.

51. - W. His. Ueber die Anfäge des peripherischen Nervensystems. Arch. f. Anat. u. Phys., anat. Abth. 1879. p. 455-482. Taf. XVII-XVIII.

52. - A. von Klein. Beiträge zur Osteologie des Schädels der Knochenfische. Mit 1 Taf. Jahreshefte d. Ver. f. vaterl. Naturk. Württemb. 35. Jahrg. 1879, p. 66126.

53. - A. Kölliker. Ueber die Entwicklung des peripherischen Nervensystems. Verh. d. phys. medic. Ges. Würzbourg. N. Folge, 13. Bd. 1879, Sitzungsber. f. 1878 , p. XIX-XX.

54. - G. SchWALBe. Das Ganglion oculo-motorii. Ein Beitrag zur vergleichenden Anatomie der Kopfnerven. Jen. Zeitshr. f. Nat. wiss. 1879, 13. Bd. 2. Heft, p. 173-268, Mit. Taf. XII-XIV.

55. - R. Wiedersheim. Morphologische Studien. 1. Heft. 3 Taf. Jena, Fischer, 1880. $8^{\circ} .85 \mathrm{~S}$.

I. Das Gehirn von Ammocotes und Petromyzon Planeri mit besonderer Berücksichtigung der spinalartigen Hirnnerven. Mit Abbildgn. p. 3-26. Aussi dans: Jen. Zeitschr. 14. Bd. 1880, p. 1-24. - V. aussi: Zool. Anz. 2. Jahrg. 1879, p. 589-592 et 3. Jahrg. 1880, p. 446-449.

III. Das Skelet und Nervensystem von Lepidosiren annectens (Protopterus annectens). Mit 2 Taf., p. 43-82.-Aussi dans: Jen.Zeitschr. 14. Bd. 1880, p. 155 192.

56. - H. Beauregard. Encéphale et nerfs craniens du Ceratodus. 1 pl. Journal de l'Anat. et de la Physiol., par Robin et Pouchet. 17 e ann. 1881, no 3 , p. 230-242. 
57. - A. VQn Klein. Beiträge zur Osteologie der Fische. 1 Taf. Jahresheft d. Ver. f. Naturk. Württemb. 37. Jahrg. 1881, p. 325-360.

58. - A. M. Marshall and W. B. Spencer. Observations on the Cranial Nerves of Scyllium. With 1 pl. Quart. Journal Microsp. Sc. Vol. 21, 1881, July, p. 469-499. V. aussi: Observations on the Cranial Nerves of Scyllium. 2 pl. Studies Biol. Labor. Owens Coll. Manchester 1886. Vol. 1, p. 87-123.

59. - Nussbaum. Ueber das anatomische Verhaltnis zwischen dem Gehörorgane und der Schwimmblase bei den Cyprinoiden. Zool. Anz. 1881, p. 552.

60. - H. Schneider. Ueber die Augenmuskelnerven der Ganoiden. Jen. Zeitschr. Bd. XV, 1881 .

61. - M. Marshall. The segmental Value of the Cranial Nerves. J pl. Journ. of Anat. a. Physiol. Vol. 16, 1882, p. 305-354.

$62^{*}$. - W. VAN WiJHe. Ueber das Visceralskelet und die Nerven des Kopfes der Ganoiden und von Ceratodus. Niederländisches Archiv für Zoologie. Bd. V. 1882. 3. Hft. p. 307-320.

63. - M. SAgemehl. Beiträge aur vergleichenden Anatomie der Fische.

I. Das Cranium von Amia calva. Mit. 1 Taf. Morphol. Jahrb. 9. Bd. 1884. 2. Heft, p. 177-228.

64. - F. Ahlborn. Ueber den Ursprung und Austritt der Hirnnerven von Petromyzon. Mit 1 Taf. Zeitshr. t. wiss. Zool. 40. Bd. 1884, 2. Hft. p. 286-308.

65. - E.-A. GöLdi. Kopfskelet und Schultergürtel von Loricaria cataphracta, Balistes capriscus und Accipenser ruthenus. Mit 3 Taf. Jena. Zeitschr. 17 Bd. 1884, 1/2 Hft.p. 401-451. V. aussi: Zool. Anz. VI. Jahrgang. 1888. No 145.

66. - A. von Klein. Beiträge zur Bildung der Schädels der Knochenfische. Mit 2 Taf. Jaheshrefte d. Ver. f. Naturk. Wurttemb., 40. Jahrg. 1884, p. 129-257. 67. - R. Ramsay Wright, J.-P. Murrich, A.-B. Macallum, T.-Mc. Kenzie. Contribution to the anatomy of Amiurus. With $8 \mathrm{pl}$. Toronto, 1884. 8 . From: Proc. Canad. Instit. Toronto. N. S. Vol. 2. No 3, p. 25l-457.

68. - M. Sagemehl. (V. No 63). III. Das Cranium der Characiniden. Mit 2 Taf. u. 1 Holzschn. Morphol. Jahrb. 1885. 10. Bd. 1. Heft. p. 1-119.

69. - J. BEARD. The system of branchial sense organs and their associated ganglia in Ichthyopsida. Quaterly Journal of microscop. science. Vol. 26, 1885, p. $85-156$.

70. - A. Froriep. Ueber Anlagen von Sinnesorganen am Facialis, Glossopharyngeus und Vagus, über die genetische Stellung des Vagus zum Hypoglossus und über die Herkunft der Zungenmuskulatur. Archiv für Anat. u. Entwicklungsgesch. 1885.

71. - A. von. Klein. (Même titre qu'au no 66). 41. Jahrg. 1885, p. 107261.

72. - Mac Murrich a. J. Playfair. The Cranial Muscles of Ania calva (L.) with a consideration of the Relations of the Post-Occipital and Hypoglossal Nerves in the Various Vertebrate Groups. 1 pi. Studies Biolog. Labor. J. Hopk. Univ. Vol. 3, 1885, No3, p. 121153.

73. - MAC MUrrich and J. Playfair. The ontogeny and phylogeny of the hypoglossal nerve. Science, 1885. Vol. 5, p. 374-375.

74. - A. von Klein. (Mème titre qu'au nº 66). 42. Jabrg. 1886. p. 205-300.

75. - J. BEARD. The Ciliary or Motor-oculi Ganglion and the ganglion of the Ophthalmicus profondus in Sharks. 5 fig. Anat. Anz. 2. Jahrg. 1887, No 18/19, p. $565-575$. 
i6. - A. Froriep. Ueber das Homologon der Chorda tympani bei niederen Wirbelthieren. Anat. Anz. 2. Jahrg. 1887, n 15, p. 486-493.

77. - A. Froriep. Bemerkungen zur Frage nach der Wirbeltheorie des Kopfskelettes. Anat. Anz. 2. Jahrg. 1887, no 27. p. 815-835.

78. - C. Gegenbaur. Die Metamerie des Kopfes und die Wirbeltheorie des Koptskelettes, im Lichte der neueren Untersuchungen betrachtet und geprüft. Morphol. Jahrbuch. 13. Bd. 1887.

79. - K. Rabl. Ueber das Gebiet des Nerves facialis. Anat. Anzeiger. 2. Jahrg. 1887, n $\mathrm{n}^{\circ}$, p. 219-227.

80. - Le Roux. Recherches sur le systeme nerveux des poissons. Av. $4 \mathrm{pl}$. Caen, 1887. $8^{\circ} 114$ p. - Extr. de la thèse dans: Revue scientif. T. 44. 1889, n० 9 . p. $278-279$.

81. - N. Goronowitsch. Das Gehirn und die Cranialnerven von Acipenser ruthenus. Morphol. Jahrbuch, 30. Bd. 1888, p. 427-574.

82. - R. Chevrel. Sur le systeme nerveux grand sympathique des poissons osseux. Compt. rend. Ac. sc. Paris, 1889. T. 107, n 12, p. 530-531.

83. - J. C. Ewart. The Cranial Nerves of Torpedo (Prelim. Note). Proc. R. Soc. London. 1890. Vol. 47, nº 289, p. 290-292.

84. - A. Froriep. Ueber die Entwicklung des Sehnerven. Mit 12 Abbildgn. Anat. Anz. 6. Jahrg. 1891, nº 6. p. 155-161.

85. - M. Sagemehl. (V. n 63). IV. Das Cranium der Cyprinoiden. Mit 2 Taf. Morphol. Jahrb. 1891, 17. Bd. 4 Heft., p. 489-595.

86. - H. B. Pollard. The lateral Line system in Siluroids. With 2 pl. Zool. Jahrb. f. Anat. u. Ontog, 1892, 5. Bd., 3-4 Heft. p. 525-551.

87. - P. Mitrophanow. Sur la formation du systeme nerceux périphérique des Vertébrés. Compt. rend. Ac. sc. Paris, 1892. T. 113, n 19, p. 659-662.

88. - R. Wiedersheim. Grundriss der vergleichenden Anatomie der Wirbelthiere. 3. Auflage, 1893.

89. - F. PInkus. Ueber einen noch nicht beschriebenen Hirnnerven des Protopterus annectens. Mit 4 Ablildgn. Anat. Anz. 1894. 9. Bd. No 18. p. 562-566. 89 bis. - F. Pinkus. Die Hirnnerven des Protopterus annectens. Mit 7 Taf. Morphol. Arb. Schwalbe, 1895, 4. Bd.2. Heft. p. 275-335 et 336-346.

90. - C. Vogt et E. Yung. Traité d'anatomie comparée pratique. T. II. Paris, 1894.

91*. - Edw.-Pн. Altis. The Cranial Muscles and Cranial and First spinal Nerves in Amia calva. Journ. of Morphol. Boston, 1895. Vol. 11, n 2, p. 485491.

92. - W. Colmange. The Unsymmetrical Distribution of the Cranial Nerves of Fishes. Journ. of Anat. and Physiol. 1895, Vol. 29 (N. S. Vol. 9) P. III. Apr. p. 352-354.

93. - J. Deyl. Ueber den Sehnerven bei Siluroiden und Acanthopsiden. Mit 5 [16] Abbildgn. Anat. Anz. 1895, 11. Bd. No 1, p. 8-16.

94.. - B. Haller. Ueber den Ursprung des Nervus vagus bei den Knochenfischen. Verhandlgn. deutsch, zool. Ges. 5. Vers. Strassbg. 1895. p. 55-61.

95. - H. B. Pollard. The Oral Cirri of Siluroids and the Origin of the Head in Vertebrates. With $2 \mathrm{pl}$. Z. Vol. Jahrb. Morph. Abth. 1895. 8. Bd. 3. Hft. p. 379-424. T. 24,25 .

96*. - B. Tiesing. Ein Beitrag zur Kenntnis der Augen -, Kiefer und 
Kiemen-Muskulatur der Haie und Rochen. 3 Taf. Jena. Zeitschr. 1895. Bd. XXX, N. F. XXIII. 1. Heft, p. 75-119, 120-126.

97. - Frank-J. Cole. The Cranial Nerves of Chimaera monstrosa. With 1 fig. Proc. Roy. Soc. Edinbg. Vol. 21. March. 49.56.

98*. - Frank J. CoLE. On the Cranial Nerves of Chimaera monstrosa with a discussion of the Lateral Line System and of the Morphology of the Chorda tympani. With 2 pl. Edinbg, Rob. Grant, Williams a. Norgate, 1896, 4 - Trans. Roy. Soc. Edinbg. Vol. 38. P. III. No 19., p. 631-675, 676-680.

99. - M. Fürbringer. Ueber die spino-occipitalen Nerven der Selachier und Holocephalen. Festschrift f. C. Gegenbaur, 1897. III. Bd. p. 349-788.

100. - N. Goronowitsch. Ueber Trigemino-Facialis-Komplex von Lota. vulgaris. Festschr. f. C. Gegenbaur, 1897. III. Bd. p. 1-44.

101. - B. Haller. Der Ursprung der Vagusgruppe bei Teleostiern. Festschr. f. C. Gegenbaur. 1897. III. Bd.p. 45101.

102. - G. Ruge. Ueber das peripherische Gebiet des Nervus facialis bei Wirbelthieren. Festschr. f. C. Gegenbaur. III. Bd. p. 193-348.

103. - C. Gegenbaur. Vergleichende Anatomie der Wirbelthiere. I. Bd. Leipzig, 1898. 


\section{EXPLICATION DE LA PLANCHE I}

Fig. 1. Squelette céphalique du Silurus glanis L., vu de profil. Fig. 2.

$A_{2+3}$ apophyses épineuses fusionnées des deuxième et troisième vertèbres.

$A_{\text {4-7 }}$ apophyses épineuses des vertèbres 4 à 7 .

abd orifice de l'alisphénoïde par lequel le nerf abducteur sort du crâne.

$A i_{1} \quad$ (voir $I$ ), arc inférieur de la première vertèbre.

$A i_{2}$ arc inférieur de la deuxième vertèbre.

$A i_{3}$ (voir $\boldsymbol{M}$ ), arc inférieur de la troisième vertèbre. (L'arc inférieur de la quatrième vertèbre n'est pas visible dans les figures, il sert de base à $\left.A i_{3}\right)$.

Ais-6 arc inférieur de la cinquième et de la sixième vertèbre.

Al alisphénoïde.

Ant os antorbital.

Art articulaire.

$A s_{1} \quad$ (voir $S$ ), arc supérieur de la première vertèbre.

$A s_{2}+3$ arcs supérieurs soudés des denxième et troisième vertèbres.

$A s_{4-5}$ arc supérieur de la quatrième et de la cinquième vertèbre.

$\mathrm{Bm}$ barbillon maxillaire.

$B S c$ barre du supra-claviculaire, appuyée sur l'occipital latéral.

$\mathrm{CHm}$ crête de l'os hyomandibulaire servant à l'origine du muscle adducteur mandibulaire profond.

Chy cérato-hyal.

$\mathrm{Cl}$ claviculaire.

Clt claustrum.

CMk cartilage de Meckel.

Co crête occipitale.

D $\boldsymbol{0}$ os dental.

Ehy épihyal.

Eth ethmoide.

Exo exoccipital.

Ff fissure frontale.

$F_{0}$ fissure occipitale.

$F p \quad$ frontal principal.

$g l$ orifice de l'os occipital latéral par lequel le nerf glossopharyngien sort du crâne.

Hhy hypohyal.

$\mathrm{Hm}$ os hyomandibulaire.

$I\left(A i_{1}\right)$ incus ou arc inférieur de la première vertèbre.

Iop interopercule.

Ln ligne nucale sur le frontal principal.

$\boldsymbol{M}\left(\mathrm{Ai}_{3}\right)$ malleus ou arc inférieur de la troisième vertèbre.

Mpt métaptérygoïde.

$M x$ os maxillaire (rudimentaire).

Nas os nasal.

$n W b$ orifice de l'occipital supérieur par lequel le nerf de WeBer sort du cràne.

$o c$ orifice de l'os occipital latéral par lequel le nerf occipital sort du crâne.

Ocs occipital supérieur.

Ol occipital latéral.

$o m+t r l$ orifice de l'alisphénoïde par lequel les nerfs oculo-noteur et trochléaire réunis sortent du crâne.

op opercule.

op orifice par lequel le nerf optique sort du crâne.

Ors orbito-sphénoïde.

$\mathrm{Pa}$ pariétal.

$P e \quad$ os pétrenx.

Pmx os prémaxillaire.

Pop préopercule.

Prf préfrontal.

Ps parasphénoïde.

Psf post-frontal. 
Qd os carré.

$R b_{1-15}$ rayons branchiostèges.

$R p_{1-15}$ rayons de la nageoire pertorale.

$S \quad$ stylo-hyal.

$S\left(A s_{1}\right)$ stapes ou arc supérieur de la première vertèbre.

$S c \quad$ supra-claviculaire.

So (3) trois os sous-orbitaires.
$S q \quad$ os squameux.

$V_{4+5}$ Corps fusionnés des quatrième et cinquième vertèbres.

$v g$ orifice de l'os occipital latéral par lequel le nerf vague sort du crâne.

$X$ osselet tubuliforme reliant l'opercule avec l'os squameux.

1 Orifice par lequel le tronc hyoüdeo-mandibulaire parvient à la face externe de l'os hyomandibulaire (voir fig. 6).

2 Orifice situé à la limite entre l'os carré et le préopercule et livrant passage à la branche mandibulaire interne du tronc hyoïdeo-mandibulaire (voir

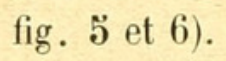

3 Orifice situé à la limite entre l'os hyo-mandibulaire et le préopercule et livrant passage au nerf hyoïdien (voir fig. 5 et 6 ). 
Nas Ant Prf Fp Ps op om+trl Psf $\mathrm{Hm} \mathrm{Sq} \quad \mathrm{A}_{2+3}$ Ol Exo $\quad$ Sc $A_{4} \mathrm{Ai}_{2} \mathrm{Ai}_{5} \quad \mathrm{~A}_{7}$

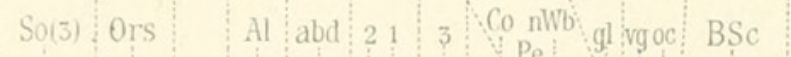

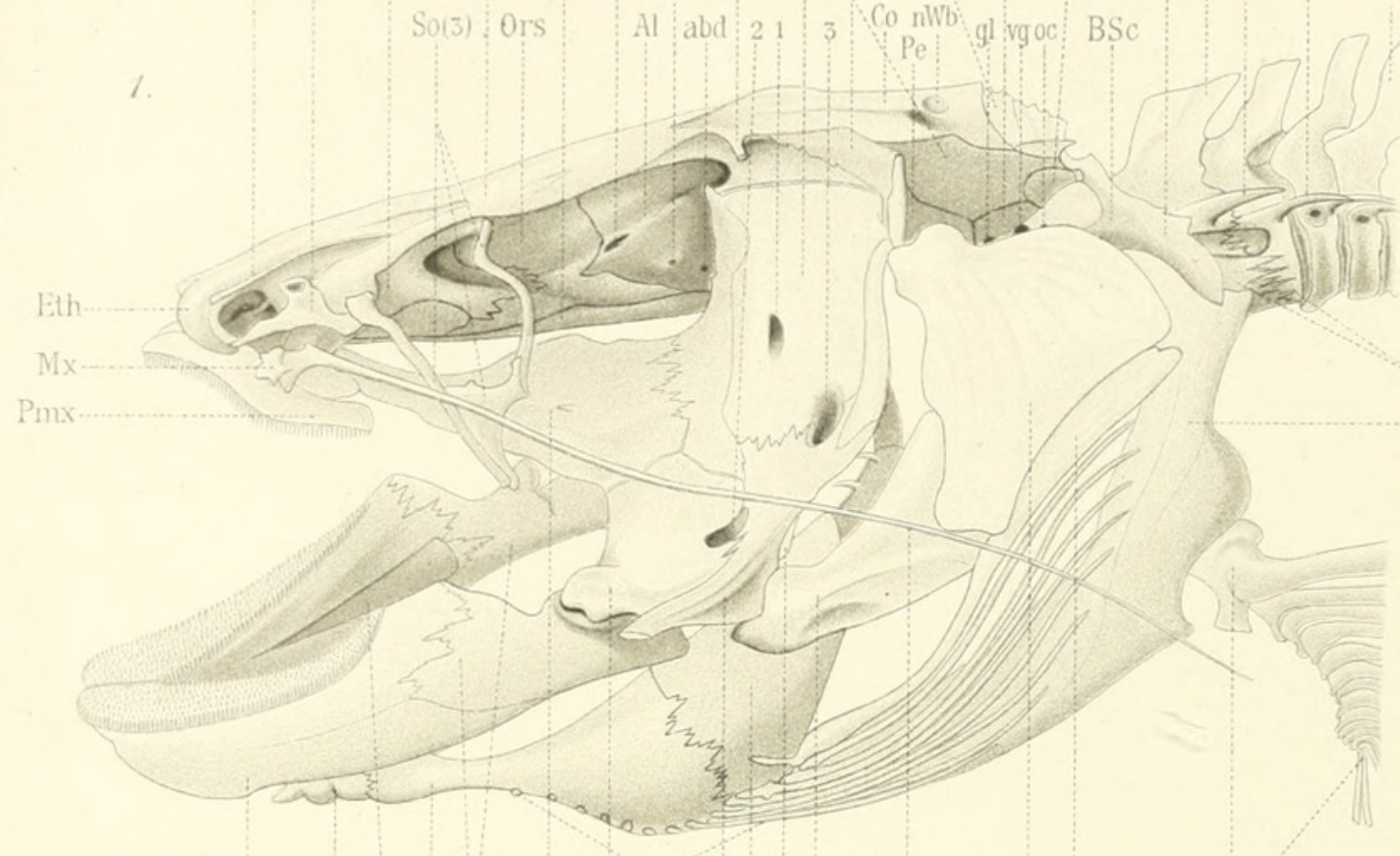

H.hy Chy $\quad \mathrm{Rb}_{1-15} \mathrm{Fhy}^{\mathrm{S}}$

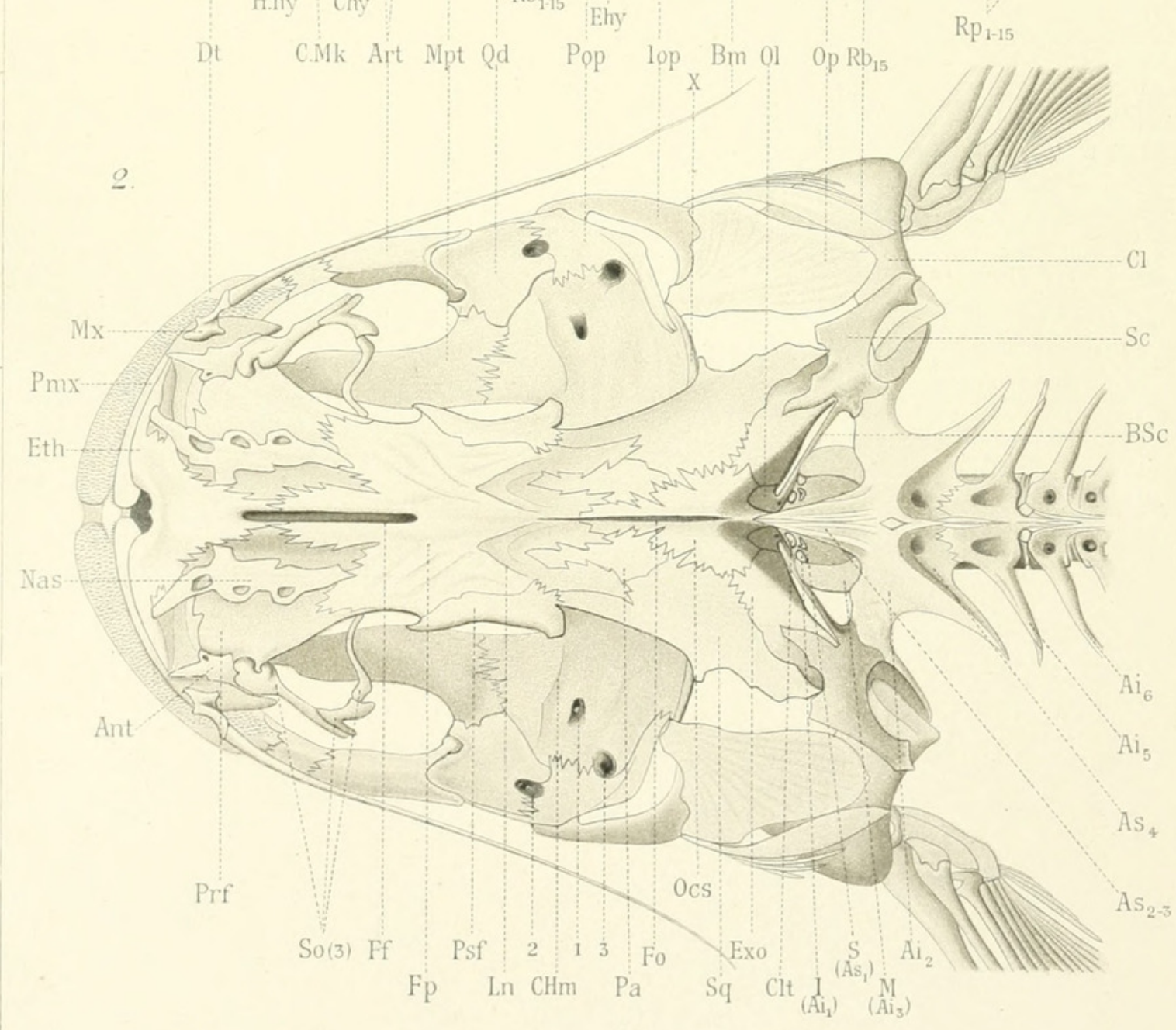


Fig. 3. Nerfs cérébraux du Silurus glanis L., vus de profil. - Les nerfs moteurs oculaires ne sont pas représentés dans cette figure. A part le nerf de Weber $(n W b)$ et les rameaux ascendants antérieurs (rasc) qui sont en noir, les branches du trijumeau-facial sont en couleur: les ophtalmiques en jaune, les maxillaires en bleu, le tronc hyoïdeo-mandibulaire en vert.

Fig. 4. Nerfs cérébraux de la moitié gauche de la tête, vus d'en haut. - Lès figures 3 et 4 ont été dessinées d'après la même préparation et se complètent l'une l'autre. Dans la figure 4, les nerfs moteurs oculaires sont figurés en rouge. Les branches du trijumeau-facial ont les mêmes couleurs que dans la figure 3 .

abd nerf abducteur.

$a c \quad$ nerf acoustique; $a c_{1}$ partie antérieure, $a c_{2}$ partie postérieure.

baT $T_{1-3}$ branche antérieure de chacun des trois troncs branchiaux du vague, ou nerf postérieur des trois premiers ares branchiaux (seul le parcours dorsal est représenté).

$b i$ branche intestinale du nerf vague.

$\mathrm{Bm}$ barbillon maxillaire(seulement la base, en pointillé).

$b p T_{1-3}$ branche postérieure de chacun des trois troncs branchiaux du vague, ou nerf antérieur des trois derniers ares branchiaux (seul le trajet dorsal est représenté).

$b s \quad$ branche superficielle du nerf latéral du vague.

cav

composant antérieur du nerf vague.

Ce cervelet.

$c p v \quad$ composant postérieur du nerf vague.

$f$ faisceau communicant entre le tronc du trijumeau et le tronc hyoïdeo-mandibulaire.

g1-8 ganglions des trois trones branchiaux du nerf vague.

ggp ganglion du nerf glosso-pharyngien. go ganglion du nerf occipital.

gp nerf glosso-pharyngien.

hmd tronc hyoïdeo-mandibulaire (facial).

hy branche hyoüdienne de $h m d$ (trajet dorsal et ventral).

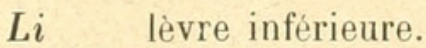

LMa lobe de la moelle allongée.

Lo bulbe olfactif (dans la fig. 3, le tractus qui le relie au prosencéphale n'est pas représenté).

Ls lèvre supérieure.

md branche mandibulaire du trone hyoïdeo-mandibulaire.

mde branche externe de $m d$.

$m d i \quad$ branche interne de $m d$.

me branche mandibulaire externe du nerf maxillaire inférieur.

Mep moelle épinière.

$m i$ branche mandibulaire interne du nerf maxillaire inférieur.

$m x i \quad$ nerf maxillaire inférieur.

$m x s$ nerf maxillaire supérieur pénétrant dans la base du barbillon maxillaire.

$N$ fosse nasale, en communication avec les deux orifices nasaux externes (contour en pointillé).

$n b p I V$ partie dorsale du nerf branch. postérieur du qualrième arc (ce nerf dépend de la branche pharyngienne inférieure). 
$n l v$

nn

nerf latéral du vague.

nerf des narines (dépend du nerf ophtalmique superficiel).

nol nerf olfactif.

$n p b \quad$ nerf postérieur du barbillon maxillaire.

$n W b$ nerf de Weber (coupé près de sa base dans la fig. 4).

0 ail.

oc nerf occipital.

om nerf oculo-moteur commun.

omds rameau de om destiné au muscle droit supérieur.

on rameau oculo-nasal de la branche ophtalmique superficielle.

op nerf optique (dans la fig. 3, la partie en pointillé est cachée par le tronc du trijumeau-facial.

opr branche ophtalmique profonde recouvrant une grande partie du nerf maxillaire supérieur.

os branche ophtalmique superficielle.

phi branche pharyngienne inférieure du vague.

pmx branche prémaxillaire du nerf maxillaire supérieur.

$r_{1}, r_{2}$ premier et deuxième rameau de la branche superficielle du nerf latéral.

$r a_{1}$

rameau alvéolaire dépendant de $m e$. rameau alvéolaire dépendant de $m i$.

rameau adducteur mandibulaire.

$\begin{array}{ll}\text { rap } & \text { rameau adducteur palatin. } \\ \text { rasc } & \text { rameaux ascendants antérieurs }\end{array}$ du trijumeau-facial.

$r c$ rameau ciliaire (dépend de on).

red rameau cardiaque du vague.

$r d v \quad$ rameau dorsal du vague.

rhpg rameau hypoglosse (dans la fig. 4, représenté seulement en partie; dépend du nerf occipital et du premier nerf spinal).

ri rameau inférieur de la branche ophtalmique superficielle.

$r l$ rameau labial dépendant du nerf maxillaire inférieur.

rof rameau operculaire du tronc hyoïdeo-mandibulaire (fac.).

rov rameau operculaire du vague.

$r r b$ rameau du tronc maxillaire destiné au muscle rétracteur du barbillon.

rs rameau supérieur de la branche ophtalmique superficielle.

$r s c l$ rameau supra-claviculaire du nerf latéral.

$s p \quad$ nerf sphéno-palatin.

to tractus olfactif.

trl nerf trochléaire sortant de l'oculo-moteur commun.

$z \quad$ (fig. 4), contour du prolongement antérieur de la cavité cranienne.

$\mathrm{I} d$-IV $v$ Branche dorsale des quatre premiers nerfs spinaux.

$\mathrm{I} v$-IV $v$ Branche ventrale des quatre premiers nerfs spinaux.

1 Rameau du nerf ophtalmique superficiel destinè à l'enveloppe oculaire et au tégument situé autour de l'œil.

2 Rameau du nerf oculo-nasal ayant même fonction que 1.

3 (fig. 4), rameau du nerf ophtalmique profond ayant mème fonction que 1 et 2.

4 (fig. 3), rameau de l'ophtalmique profond ayant même fonction que 1, 2 et 3. 

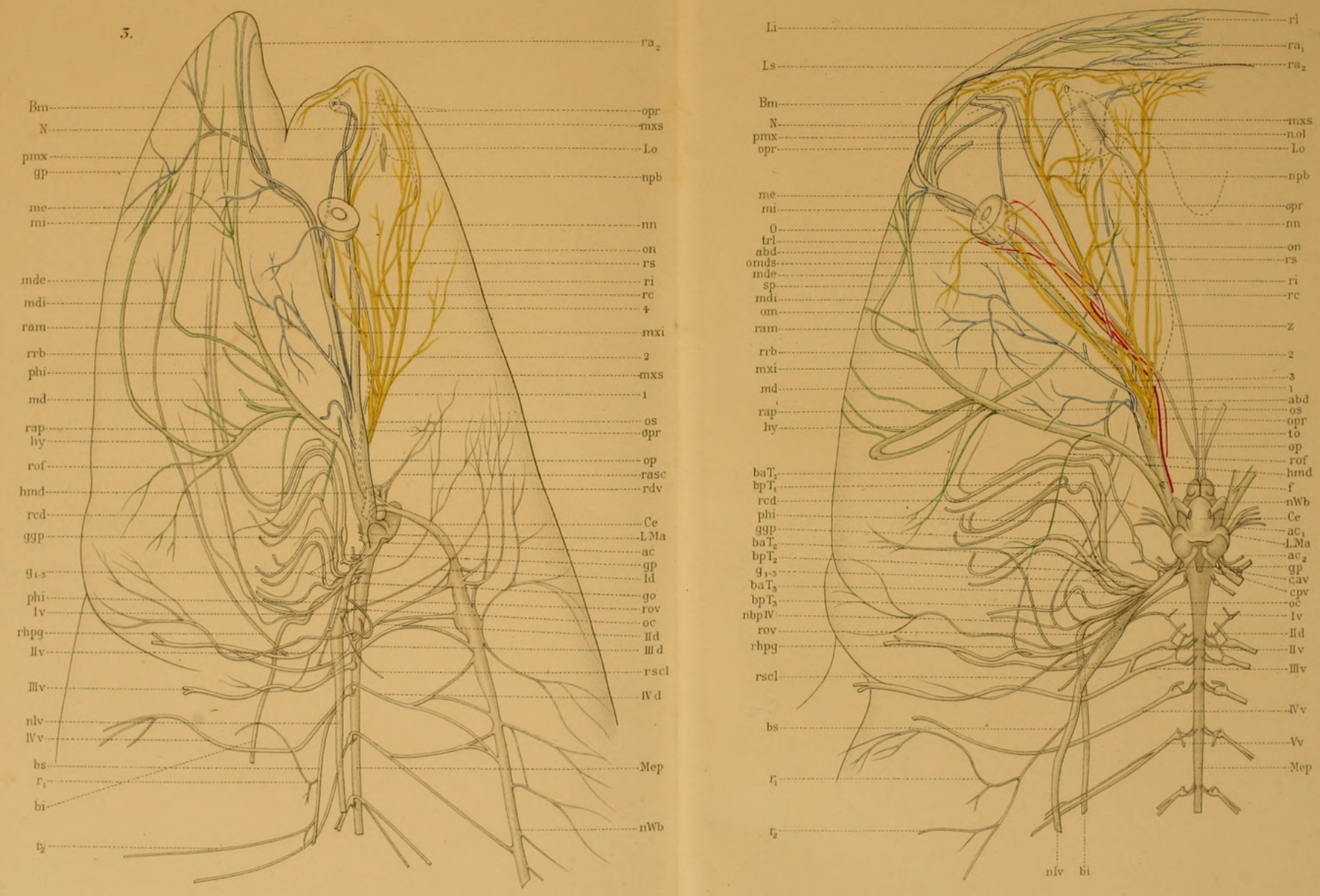


\section{EXPLICATION DE LA PLANCHE 3}

Dans cette planche, les muscles sont en rose, les tendons et les ligaments en bleu, et les nerfs en jaune.

Les désignations concernant les nerfs sont en saractères droits, mais sans majuscule (nerfs) ; les désignations de muscles sont en italique et commencent par une majuscule (Muscles); les autres sont en caractères droits et commencent par une majuscule $(0 \mathrm{~s})$.

Fig. ら̈. Tête de Silurus glanis L., vue d'en haut. Du côté gauche, on a seulement enlevé la peau; du côté droit, on voit les muscles sitnés audessous de ceux représentés à gauche.

Fig. 6. Vue dorsale de la moitié gauche de la têtè, après que l'on a enlevé le couvercle cranien et le muscle élévateur palatin. Les os squameux $(\mathrm{Sq})$, post-frontal (Psf), alisphénoïde (Al) et orbito-sphénoïde (Ors) sont donc vus en section horizontale.

Fig. 7. Partie dorsale de l'appareil branchial du côté droit, vue d'en haut.

Fig. 8. Partie dorsale de l'appareil branchial du côté droit, vue d'en bas. Le revêtement cutané a été enlevé jusqu'à la partie libre des arcs.

Fig. 9. Moitié gauche de la tête, face ventrale. La peau seule est enlevée afin de faire voir les muscles superficiels. Les pointillés représentent des parties situées plus profondément.

Fig. 10. Musculature ventrale profonde de la tête, vue d'en bas.

Cóté gauche : on a enlevé le génio-hyoïdien, le hyo-hyoïdien inférieur, la plus grande partie du cérato-hyal (Chy) et coupé près de leur base les six premiers rayons branchiostèges $(\mathrm{Rb})$; le contour de la partie antérieure de la clavicule est représenté par le pointillé noir, afin de rendre visibles les parties que cet os recouvre ventralement.

Cóté droit: on a enlevé la moitié droite de l'uro-hyal, le sternohyoïdien et les deux pharyngo-claviculaires, afin de montrer la musculature la plus profonde des extrémités ventrales des arcs viscéraux.

$A m_{1}$ rameau du nerf maxillaire inférieur destiné au barbillon mandibulaire antérieur. partie latèrale de $A m_{1}$. tendon de la partie $a$ de $A m_{1}$. nerf abducteur. adducteur hyomandibulaire. alisphénoïde. adducteur mandibulaire, portion superficielle (dans la fig. 9 , on n'en voit que le bord inférieur).
$A m_{2}$ adducteur mandibulaire, por-

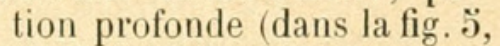
seulement la partie antérieure).

$A m_{2}$ t tendon de $\mathrm{A} m_{2}$.

$A m p$ prolongement de $A m$ s'insérant sur le cartilage de MEgKel.

Ant os antorbital.

Ao adducteur operculaire.

Art articulaire. 
rameau peaucier et moteur (voir rim) du nerf maxillaire inférieur.

$b$ partie médiale de l'adducteur mandibulaire supericiel.

$\mathrm{Ba}$ barbillon mandibulaire antérieur.

ba $T_{1-3}$ branche antérieure des trois troncs branchiaux du nerf vague, ou nerf postérieur des trois premiers arcs branchiaux.

bi branche intestinale du nerf vague.

Bp barbillon mandibulaire postérieur.

$\mathrm{Bp}_{1-3}$ branche postérieure des trois troncs branchiaux du nerf vague, ou nerf antérieur des trois derniers arcs branchiaux.

$b_{t} \quad$ tendon de la partie médiale $(b)$ de $A m_{1}$.

$b_{t}+d_{t}$ lame tendineuse formée de la réunion de. $b_{t}$ et $d_{t}$, redevient musculeuse en avant (fig. 6, $A m p$ ).

C copule.

c rameau du nerf maxillaire inférieur innervant le muscle génio-hyoïdien.

CbrI-IV cérato-branchiaux des quatre arcs (dans les fig. 7 et 8 , ils sont coupés horizontalement près de leur extrémité postérieure).

Ceth cartilage médian de l'ethmoïde. Chy cérato-hyal (dans la fig. 9, contour en pointillé).

Cl os claviculaire (dans la fig. 10 , contour en pointillé).

Cit claustrum.

CMk cartilage de Meckes.

ConsI V constricteur du quatrième arc branchial (dans la fig. 7, il esi caché par l'épibranchial IV : dans la fig. 8, sa partie latérale est coupée horizontalement).

Cph constricteur pharyngien (dans la fig. 8, sa partie latérale est coupée horizontalement comme les arcs branchiaux).

cpv composant postérieur du nerf vague.

$c_{t} \quad$ tendon de la partie latérale du muscle adducteur mandibulaire profond.
De

Di muscle droit externe de foil.

Di muscle droit interne de l'œil.

$D_{0 a} \quad$ dilatateur operculaire, partie antérieure.

$D_{o_{p}} \quad$ dilatateur operculaire, partie postérieure.

Dot tendon du Do.

Ds muscle droit supérieur de l'œil.

Dt os dental.

e rameau peaucier du nerf maxillaire inférieur.

EbrI-IV épibranchiaux des quatre arcs.

Ec partie supérieure de l'élévateur claviculaire.

Ehy épihyal.

Eib extenseur inférieur du barbillon maxillaire.

Eibo origine de Eib.

$E l b_{1-4}$ élévateurs branch. des quatre arcs (dans la fig. 7 , on voit les parties supérieures des quatre muscles, mais détachées des os du crâne sur lesquels elles naissent; dans la fig. 8 , on ne voit que la partie postérieure et inférieure des deux muscles postérieurs).

Eo élévateur operculaire.

Ep élévateur palatin.

Esb extenseur supérieur du bar. billon maxillaire.

$E s b_{o}$ origine de $E s b$.

Eth os ethmoïde.

$f A m_{1}$ fascia de l'adducteur mandibulaire superficiel prolongé en avant.

Fp frontal principal.

Gh muscle génio-hyoïdien.

gp nerf glosso-pharyngien.

HbrI-III hypobranchiaux des trois premiers ares.

Hhi hyo-hyoïdien inférieur (le pointillé rouve limite la partie recouverte par le muscle génio-hyoïdien).

Hhit tendon de Hhi.

$H h i_{t} 1$ partie de $H h i_{t}$ s'insérant sur le hypohyal de l'autre côté.

Hhs hyo-hyoïdien supérieur.

Hhs origine de Hhs à la face interne de l'appareil operculaire. 
Hhy hypohyal (dans la fig. 9, contour en pointillé).

$\mathrm{Hm}$ os hyomandibulaire.

hmd tronc hyoïdeo-mandibulaire (facial).

hy branche hyoïdienne du hmd (dans la fig. 9, son parcours dorsal est en pointillé).

Im muscle intermandibulaire.

Iop interopercule.

L ligament entre l'articulaire et le bord de la mâchoire supérieure.

LIop ligament reliant l'interopercule à la région angulaire de l'articulaire.

LMx ligament reliant l'os maxillaire au prémaxillaire et à l'ethmoide.

LUhy ligament reliant l'uro-hyal à l'hypohyal.

md branche mandibulaire du tronc hyoïdeo-mandibulaire (hmd).

mde branche externe de md (dans la fig. 9, le pointillé indique le trajet sur la face supérieure de l'arcade palatine).

mdi branche interne de md (dans la fig. 9, son passage sous le cartilage de Meckes est indiqué en pointillé).

$M d l$ muscle dorso-latéral (prolongement céphalique).

me branche mandibulaire externe du nerf maxillaire inférieur.

mi branche mandibulaire interne du nerf maxillaire inférieur (dans la fig. 9, le pointillé indique le passage contre la face interne de l'os dental).

Mphi muscle reliant les extrémités postérieures du cérato-branchial IV et de l'os pharyngien inférieur.

Mpt métaptérygoïde.

Mvl partie antérieure du muscle ventro-latéral (s'insère sur la face postérieure de la clavicule).

Mx os maxillaire rudimentaire.

$\mathrm{mxi}$ nerf maxillaire inférieur.

mxs nerf maxillaire supérieur.

N cavité logeant la cupule nasale. Nas os nasal.
nbplV nerf branchial postérieur du quatrième are (depend de phi).

$O d_{1}$ oblique dorsal inséré sur le premier arc branchial.

$O d_{a} \quad$ obliques dorsaux, partie antérieure.

$O d_{p} \quad$ obliques dorsaux, partie postérieure.

Oi muscle oblique inférieur de l'eil.

Oio orifice interne du canal de l'optique dans l'alisphénoïde.

Op opercule.

opr branche ophtalmique profonde.

Ors orbito-sphénoïde.

Os muscle oblique supérieur de l'œil.

Pah muscle pharyngo-arcuo-hyoïdien.

$\mathrm{Pah}_{t}$ tendon du Pah.

PbrI+II extrémités médiales des pharyngo-branchiaux I et II soudées en une pièce commune.

PbrI-IV pharyngo-branch. des quatre ares.

$\mathrm{Pce}_{i}$ partie médiale de l'insertion du pharyngo-claviculaire externe $(\boldsymbol{P} c e)$.

$\mathrm{Pce}_{0}$ origine du Pce.

Pci pharyngo-claviculaire interne.

$\mathrm{Pci}_{i} \quad$ partie postérieure de l'insertion du Pci.

$\mathrm{Pci}_{0}$ origine du $\mathrm{Pci}$.

Phi os pharyngien inférieur (dans la fig. 8, coupé horizontalement près de son extrémité supérieure; dans la fig. 10, il est en grande partie recouvert par des muscles).

phi branche pharyngienne inférieure du vague.

Pht muscle pharyngien transverse.

Pop préopercule.

$\mathrm{Pph}$ plaque pharyngienne (supposée transparente).

Prf préfrontal.

Psf post-frontal.

Qd os carré.

ras rameau alvéolaire dépendant de la branche externe (me) du maxillaire inférieur. 
$\mathrm{ra}_{2}$ rameau alvéolaire dépendant de la branche interne (mi) du maxillaire inférieur.

ram rameau adducteur mandibulaire.

Rb1-15 rayons branchiostèges.

Rba rétracteur branchial antérieur (sa partie postérieure qui s'attache en arrière sur l'exoccipital est représentée en pointillé, afin de rendre visibje ce qui est situé audessous).

rbg rameau branchial du nerf glosso-pharyngien se prolongeant dans le pharyngoarcuo-hyoïdien .

Rbm rélracteur du barbillon maxillaire.

$R b p$ rétracteur branchial postérieur (son extrémité postérieure a été simplement détachée de l'exoccipital sur lequel elle prend naissance.

rbv rameaux branchiaux du vague se prolongeant dans le pharyngo-arcuo-hyoüdien.

reib rameau du tronc hyoïdeo-mandibulaire destiné à l'extenseur inférieur du barbillon.

resb rameau du tronc hyoïdeo-man dibulaire destiné à l'extenseur supérieur du barbil!on.

rhpg rameau hypoglosse (dépend du nerf occipital et du premier nerf spinal).

ri partie du rameau inférieur de la branche ophtalmique superficielle.

rim rameau du nerf maxillaire inférieur allant au muscle intermandibulaire. rof

rov

rib

rros

$\operatorname{rrp}$

rs

rtd

Sc supra-claviculaire.

sp partie du nerf sphéno-palatin.

Sq os squameux).

Sth muscle sterno-hyoïdien.

Stho origine du Sth sur la face supérieure de la clavicule (Cl).

Td transverse dorsal moitié dr.).

trl nerf trochléaire.

Tv transverse ventral.

Uhy uro-hyal (moitié gauche).

X osselet tubuliforme entre le squameux et l'opercule.

y paroi membraneuse de la cavité branchiale.

1 orifice par lequel le tronc hyoïdeo-mandibulaire parvient à la face externe de l'os hyomandibulaire.

2 orifice livrant passage à la branche mandibulaire interne du tronc hyoüdeomandibulaire (fig. 5ँ, mdi).

3 orifice livrant passage au nerf hyoïdien (fig. こँ, hy). 

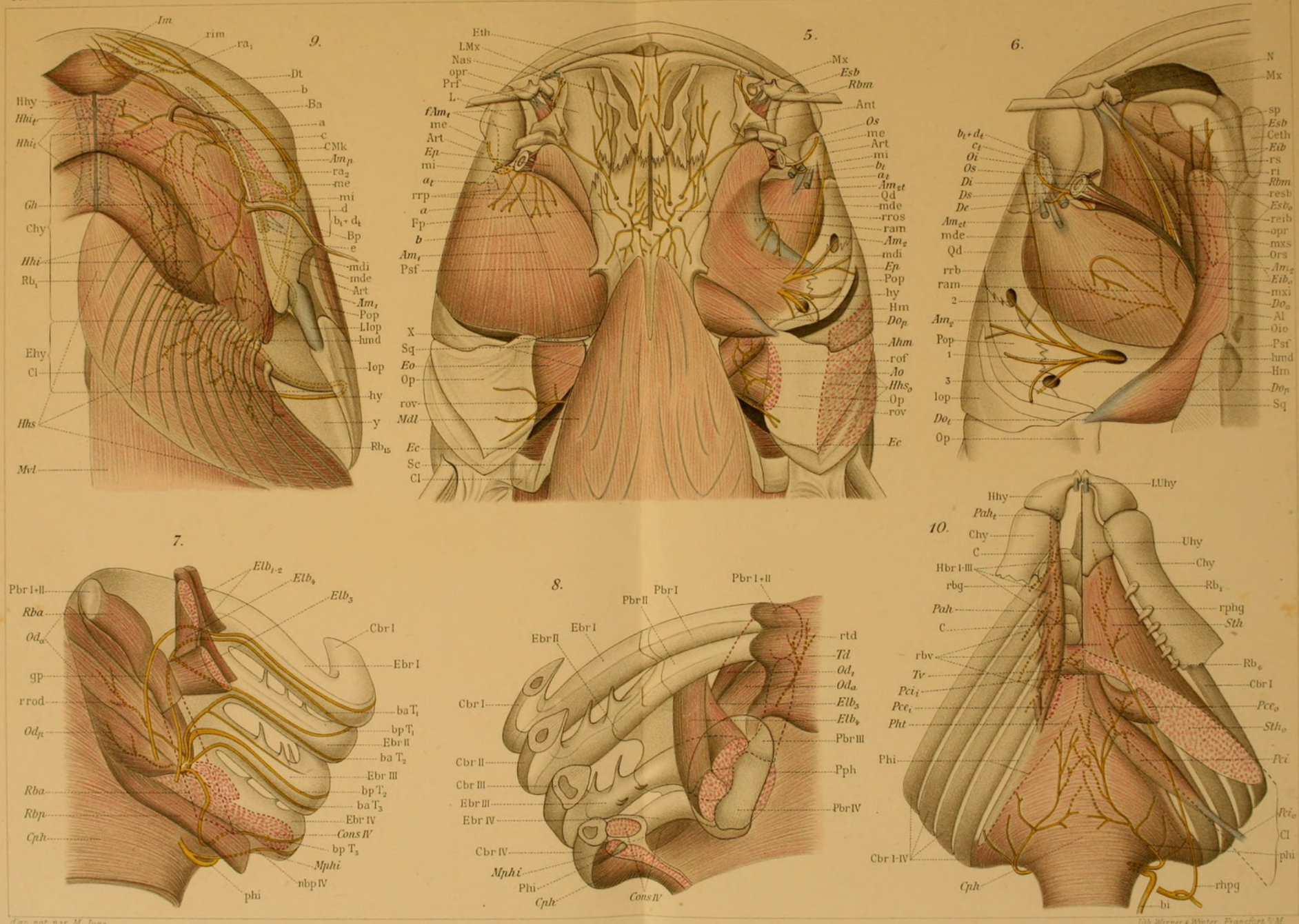


\section{$2 \mathrm{BHL}$ Biodiversity Heritage Library}

Juge, Marc. 1899. "Recherches sur les nerfs cérébraux et la musculature céphalique de Silurus glanis." Revue suisse de zoologie 6(1), 1-171. https://doi.org/10.5962/bhl.part.75147.

View This Item Online: $\underline{\text { https://www.biodiversitylibrary.org/item/39595 }}$

DOI: https://doi.org/10.5962/bhl.part.75147

Permalink: https://www.biodiversitylibrary.org/partpdf/75147

\section{Holding Institution}

MBLWHOI Library

\section{Sponsored by}

MBLWHOI Library

\section{Copyright \& Reuse}

Copyright Status: NOT_IN_COPYRIGHT

This document was created from content at the Biodiversity Heritage Library, the world's largest open access digital library for biodiversity literature and archives. Visit BHL at https://www.biodiversitylibrary.org. 